\title{
Evolutionary origin of the human pair-bond - the adaptive significance of male-female relationships in wild Assamese macaques (Macaca assamensis), Thailand
}

\author{
Dissertation \\ zur Erlangung des mathematisch-naturwissenschaftlichen Doktorgrades \\ „Doctor rerum naturalium“ \\ der Georg-August-Universität Göttingen \\ im Promotionsprogramm Behavior and Cognition (BeCog) \\ der Georg-August University School of Science (GAUSS)
}

vorgelegt von

Christine Barbara Haunhorst

aus Bonn 


\section{Betreuungsausschuss}

Julia Ostner, Verhaltensökologie, Universität Göttingen

Dietmar Zinner, Kognitive Ethologie. Deutsches Primatenzentrum

Tanya Behne, Kognitive Entwicklungspsychologie, Universität Göttingen

\section{Mitglieder der Prüfungskommission}

Referentin: Julia Ostner, Verhaltensökologie, Universität Göttingen

Korreferent: Dietmar Zinner, Kognitive Ethologie, Deutsches Primatenzentrum

Ggf. 2. Korreferent/in:

Weitere Mitglieder der Prüfungskommission:

Tanya Behne, Kognitive Entwicklungspsychologie, Universität Göttingen

Oliver Schülke, Verhaltensökologie, Universität Göttingen

Peter Kappeler, Verhaltensökologie und Soziobiologie, Universität Göttingen

Bernhard Fink, Biologische Persönlichkeitspsychologie, Universität Göttingen

Tag der mündlichen Prüfung: 10. August 2016 
Dedicated to a wise person

Without whom I could not have done it

„Field work never works out as planned;

monkeys live in trees and are just hard to observe - so relax!"

Anja Ebenau 



\section{TABLE OF CONTENTS}

SUMMARY

ZUSAMMENFASSUNG 3

GENERAL INTRODUCTION $\quad 7$

OPPOSITE-SEX SOCIAL BONDS IN MULTI-MALE PRIMATE GROUPS 10

PATERNAL CARE

FEMALE COMPETITION

EVOLUTION OF PERMANENT PAIR-BONDS IN PRIMATES 21

STUDY SPECIES

THESIS AIMS

$\begin{array}{ll}\text { CHAPTER } 1 & 27\end{array}$

OPPOSITE-SEX SOCIAL BONDING IN WILD ASSAMESE MACAQUES

Christine B. Haunhorst, Oliver Schülke \& Julia Ostner

CHAPTER 2

SOCIAL BONDS WITH MALES LOWER THE COSTS OF COMPETITION FOR WILD FEMALE ASSAMESE MACAQUES Christine B. Haunhorst, Marlies Heesen, Julia Ostner \& Oliver Schülke

\section{CHAPTER 3}

FEMALE COMPETITION FOR 'POWER' AND ‘COMMITMENT’ IN MALE PARTNERS - ASSAMESE MACAQUES AS A TEST CASE

Christine B. Haunhorst, Michael Heistermann, Ines Fürtbauer, Oliver Schülke \& Julia

Ostner

GENERAL DISCUSSION

THE ADAPTIVE SIGNIFICANCE OF OPPOSITE-SEX SOCIAL BONDS FOR ASSAMESE MACAQUES

SPECIES COMPARISON

ASSAMESE MACAQUES AS MODEL ORGANISM FOR THE EVOLUTION OF HUMAN PAIRBONDS

CONCLUSIONS AND FUTURE DIRECTIONS

REFERENCES $\quad 107$

ACKNOWLEDGEMENTS $\quad 123$

CURRICULUM VITAE $\quad 127$

DECLARATION 129 



\section{Summary}

The patterns of social interactions and the resulting relationships between members of a social group shape the social structure of animal societies. In large primate groups, both sexes potentially form differentiated social relationships with each other, which can result in reproductive benefits. Evidence is accumulating on how important same-sex social relationships might be for the philopatric, and the dispersing sex. Opposite-sex relationships have so far been only studied in a handful of primate species and broad insight on the benefits both sexes derive is rare. This is surprising, considering that the behavior of extant non-human primates may help to unravel significant parts of the evolution of humans. The long-lasting pairbond may be the core feature shaping human societies. How long-lasting pair-bonds in humans evolved is still under debate, as well as the evolution of social monogamy in nonhuman primates. To draw conclusions of sequential evolution of this particular trait, we need to understand the patterns of relationships between the sexes, the benefits both partners may derive and the costs they are willing to take.

The aim of my study was to elucidate the adaptive significance of opposite-sex affiliative relationships in wild Assamese macaques (Macaca assamensis) with its implications for the evolution of human pair-bonds. I explored the patterns, stability and equitability of opposite-sex affiliative relationships, assessed the benefits, females derive from affiliating with particular males and the occurrence of female competition for male partners. I used behavioral data ( 10,000 hrs) on all adult males and females of two habituated groups across three nonconsecutive years collected at Phu Khieo Wildlife Sanctuary, in northeastern Thailand, combined with fecal glucocorticoid analysis and feeding data on females. I calculated dyadic composite sociality indices (CSI) to assess the strength of dyadic affiliative relationships between males and females, and group-wide differentiation at certain observation periods. In all following analyses, I tested how the strength of a dyadic affiliative relationship (measured by CSI) effected the dyad's behavior. I assessed the stability and equitability of affiliative relationships to define social bonds using established indices (partner stability index, PSI; grooming index, Gl). I used data on aggressive conflicts to assess male coalitionary support for females, and focal tree observations to estimate co-feeding and food intake rates of females. I controlled for individual preferences when necessary, by using statistical within subject designs. I defined competitive situations when several females shared the same male as their top partner and used fecal glucocorticoid metabolite levels to explore female costs, in terms of stress.

I found opposite-sex affiliated relationships in Assamese macaques highly differentiated across the group, with only a third of dyads featuring strength above the group's 


\section{Summary}

average CSI. In most cases, both partners of a dyad did have different individuals as top partners and only few affiliative relationships were mutually the strongest. Only a few males were top partner of several different females at a time, while other males were not top partner of any female. The strongest affiliative relationships were stable across reproductive seasons, as well as non-consecutive years. The stronger the affiliative relationship the higher was the chance of grooming reciprocation within a reproductive season, but females did overall groom males more than vice versa and the CSI did not have an effect on grooming symmetry within a dyad. However, I found that the strength of an affiliative relationship reduced the amount of aggression a female received by a particular male, and males did support females more, the stronger the affiliative relationship was. Females' co-feeding with males, and ingestion rates in the presence of males, were positively predicted by the strength of the affiliative relationship. Female competition for male partners became apparent by assortative bonding based on similarity of dominance rank and the female dominance rank being predictive of the strength of affiliative relationships in competitive situations. Contradictory to my prediction, I found elevated fecal glucocorticoid metabolite levels for females that managed to build the strongest bond to a male within a competitive situation. Dominance rank and time spent with immatures did independently predict the male's "attractiveness" to females.

In summary, female Assamese macaques form differentiated affiliative relationships with the opposite sex that are stable over time and more or less equitable, which are the main features of a social bond. Females gained benefits, in terms of lowered aggression, agonistic support and lowered feeding competition from social bonds with males that may have direct impact on female reproductive success. Accordingly, females did compete for access to the valuable resource 'male'. To females, those males were most attractive that had the highest potential to increase a female's reproductive fitness by access to valuable resources (high dominance rank) or the potential for paternal care (time spent with immatures).

My study does not only contribute to the existing knowledge on opposite-sex relationships but provides detailed assessment of the costs and benefits females face by forming social bonds with males. To my knowledge, this was the first study to show increased ingestion rates based on dyadic relationship patterns. Studies on female competition for male partners are scarce and evidence for the mechanisms of female competition is in need. In that respect, my study sheds some light on the distinct features and significance of opposite-sex social bonds for females in multimale-multifemale primate groups with its implications for the evolutionary origin of the human pair-bond. 


\section{Zusammenfassung}

Die Muster sozialer Interaktionen und daraus entstehende Beziehungen zwischen Gruppenmitgliedern bestimmen die soziale Struktur tierischer Gesellschaften. In großen Gruppen von Primaten formen prinzipiell beide Geschlechter soziale Beziehungen miteinander. Es ist bekannt, dass aus starken Sozialbeziehungen innerhalb einer Gruppe reproduktive Vorteile für die einzelnen Individuen entstehen können. Es häufen sich die Belege darüber, wie wichtig gleichgeschlechtliche Beziehungen sowohl für das philopatrische als auch das abwandernde Geschlecht sind. Andererseits wurden andersgeschlechtliche Beziehungen bisher nur in wenigen Arten untersucht und weitreichende Erkenntnisse bezüglich der daraus entstehenden Vorteile für beide Geschlechter sind unzureichend. Dies ist überraschend, wenn man bedenkt, dass das Verhalten heute noch existierender nicht-menschlicher Primaten Hinweise auf die Evolution des Menschen liefern könnte und solche Vergleiche auch in zunehmendem Maße herangezogen werden. Wie und weshalb sich speziell beim Menschen eine langanhaltende Paarbindung innerhalb großer Gruppen entwickelte wird noch immer diskutiert. Auch die Evolution von Monogamie bei Affen gibt bis heute Anlass zu kontroversen Diskussionen und es herrscht generell Uneinigkeit zwischen verschieden Theorien. Um Schlüsse über die sequentielle Evolution dieses bestimmten Merkmals ziehen zu können, müssen wir den Aufbau andersgeschlechtlicher Beziehungen verstehen, sowie die Vorteile, die beide Partner davon haben und die Kosten, die diese gewillt sind einzugehen.

Meine Studie sollte die adaptive Signifikanz andersgeschlechtlicher Beziehungen bei wilden Assam-Makaken (Macaca assamensis) untersuchen. Aus den daraus gezogenen Schlüssen, wollte ich mögliche Ursprünge der Evolution der menschlichen Paarbindung beleuchten. Zuerst habe ich die Variabilität, Stabilität und Ausgeglichenheit andersgeschlechtlicher Beziehungen untersucht, um eine umfangreiche Definition und Zuordnung solcher Beziehungen zu liefern. Als nächstes habe ich genauer die Vorteile untersucht, die Weibchen durch eine Beziehung mit einem Männchen erhalten, um die Gründe für das Auftreten solcher Beziehungen näher zu beleuchten. Zum Schluss habe ich untersucht, ob Weibchen um den Zugang zu männlichen Partnern konkurrieren, um die Signifikanz dieser Beziehungen für die Weibchen genauer festzustellen. Ich habe meine Studie in Phu Khieo Wildlife Sanctuary im Nordosten Thailands durchgeführt. Ich habe kontinuierliche Verhaltensdaten ( 10000 h) an allen erwachsenen Männchen und Weibchen von zwei an Menschen gewöhnten Gruppen über einen Zeitraum von drei separaten Jahren mit Glucocorticoid Analysen (aus Kot) und Fressdaten von Weibchen kombiniert. Ich habe dyadische Sozial-Indices (dyadic composite sociality index; CSI) berechnet, um die Stärke dyadischer Beziehungen zwischen Männchen und Weibchen zu bestimmen, sowie die 
gruppenweite Differenzierung in bestimmten Beobachtungszeiträumen. In allen folgenden Analysen testete ich den Effekt der Stärke einer dyadischen Beziehung (gemessen als CSI) auf das Verhalten innerhalb dieser Dyade. Meine Haupthypothese besagte, dass je stärker die Beziehung, desto stabiler und ausgeglichener sollte sie sein, sowie desto mehr Vorteile sollten die Weibchen von der Beziehung mit einem Männchen haben. Ich habe die Stabilität und Ausgeglichenheit von Beziehungen mit Hilfe etablierter Indices (Partner Stabilitäts-Index, PSI; Fellpflege-Index, GI) bestimmt, um echte soziale Bindungen zu definieren. Ich habe Daten über aggressive Konflikte zwischen Weibchen und anderen Gruppenmitgliedern genutzt, um das Vorkommen von männlicher Unterstützung zugunsten bestimmter Weibchen zu erfassen. Außerdem nutzte ich Beobachtungen des Fressverhaltens von Weibchen, um einerseits abzuschätzen, ob die Beziehungsstärke in einer Dyade die Toleranz von Männchen gegenüber Weibchen im Futter-Kontext beeinflusst, als auch ob sie die Ingestionsraten von Weibchen in Anwesenheit von Männchen beeinflusst. Eine Konkurrenzsituation habe ich definiert, wenn ein Männchen zur gleichen Zeit der stärkste Partner mehrerer Weibchen war. Ich nutzte Glucocorticoid Level um die für Weibchen entstehenden Kosten (in Form von Stress) in einer Konkurrenzsituation abzuschätzen. Ich habe die aktuellsten statistischen Methoden (in Form von Generalized Linear Mixed Models) angewandt, um für ökologische Faktoren zu kontrollieren, die das Verhalten von Tieren maßgeblich beeinflussen können. An gegebenen Stellen habe ich statistische Designs benutzt, die zwischen individuellen Präferenzen und genereller Sozialität jeden einzelnen Individuums unterschieden.

Meine Ergebnisse lieferten ein sehr umfangreiches Bild über Aufbau und Struktur, sowie über die Gründe und Signifikanz heterosexueller Beziehungen in Assam-Makaken. Andersgeschlechtliche Beziehungen in Assam-Makaken sind innerhalb der Gruppe stark differenziert. Die Mehrzahl der Beziehungen war als schwach bis nicht vorhanden einzuordnen, während nur $30 \%$ als überdurchschnittlich definiert werden konnten und lediglich $10 \%$ als besonders stark. Erste Anzeichen von Konkurrenz zwischen Weibchen waren schon daran zu erkennen, dass einige wenige Männchen zum selben Zeitpunkt die stärksten Partner mehrerer Weibchen waren, während viele Männchen von keinem einzigen Weibchen präferiert wurden. Die stärksten Beziehungen waren einerseits unabhängig von den Reproduktionsphasen des Weibchens stabil innerhalb eines Jahres, als auch über mehrere Jahre, solange die Männchen in den Gruppen verweilten. Je stärker eine Beziehung war, desto höher war auch die Chance, dass Fellpflege innerhalb einer Beobachtungsperiode erwidert wurde. Andererseits waren Weibchen grundsätzlich aktiver bei der Fellpflege als Männchen. Auch die Stärke einer Beziehung konnte diese generelle Unausgeglichenheit nicht ändern. Abgesehen davon fand ich jedoch heraus, dass die Stärke der Beziehung die Aggression innerhalb dieser Dyade verringerte, wenn die Zeit, die das Paar miteinander verbrachte als Faktor in Betracht gezogen wurde. Außerdem unterstützten Männchen solche Weibchen in Konflikten mit Gruppenmitgliedern mehr, je stärker ihre Beziehung zu diesen war. Die Stärke 
der Beziehung beeinflusste auch positiv, wie oft Weibchen mit bestimmten Männchen beim Fressen beobachtet wurden und wie hoch die Ingestionsraten in Anwesenheit eines Männchens waren. Konkurrenz zwischen Weibchen um männliche Partner ließ sich anhand von Ordnung nach Dominanz unter den Weibchen innerhalb einer Konkurrenzsituation ableiten. Entgegen meiner initialen Vorhersage zeigten Weibchen, die die stärkste Bindung zu einem Männchen innerhalb einer Konkurrenzsituation aufbauen konnten, erhöhte Glucocorticoid Level. Generell waren solche Männchen attraktiver für Weibchen, die entweder dominant waren und/oder viel Zeit mit Juvenilen verbrachten.

Zusammenfassend bilden weibliche Assam-Makaken differenzierte Beziehungen mit dem anderen Geschlecht. Die stärksten Beziehungen sind über die Zeit stabil und mehr oder weniger ausgeglichen. Dies sind die Grundvoraussetzungen für eine echte soziale Bindung. Weibchen ziehen potentiell große Vorteile aus der Beziehung, indem sie einerseits weniger Aggression von einem Männchen erwarten müssen, andererseits Unterstützung in Konflikten mit anderen erwachsenen Männchen erhalten, sowie die Konkurrenz um Futter abgeschwächt wird. All diese Faktoren können einen positiven Einfluss auf die Reproduktionsrate von Weibchen haben. Entsprechend konkurrierten Weibchen um die wertvolle „Ressource“ Männchen. Weibchen konkurrierten vor allem um solche Männchen, die ihnen den meisten Schutz bieten können, bzw. Zugang zu den meisten Ressourcen verschaffen können (hohe Dominanz) oder um solche, die Potential zum guten Vater zeigten (viel Zeit mit Juvenilen).

Meine Studie trägt nicht nur zu dem schon vorhandenen Wissen über andersgeschlechtliche Beziehungen in großen Primaten-Gruppen bei, sondern liefert detaillierte Ergebnisse über die Kosten und Nutzen, die Weibchen aus sozialen Bindungen mit Männchen ziehen können. Meines Wissens war dies die erste Studie, die einen Einfluss von Beziehungsstärke auf Ingestionsraten zeigen konnte. Studien über die Konkurrenz zwischen Weibchen über männliche Partner sind außerdem selten und die zugrundeliegenden Mechanismen bisher weitgehend unbekannt. Meine Studie konnte dazu beitragen, die distinkten Eigenschaften andersgeschlechtlicher Beziehungen in beid-geschlechtlichen Primaten-Gruppen zu beleuchten und die adaptive Signifikanz für Weibchen weiter aufklären, inklusive der daraus abzuleitenden Vorhersagen für den evolutionären Ursprung menschlicher Paar-Bindung. 


\section{General Introduction}

Researchers strive to elucidate the development of human societies to reveal our evolutionary origins. The human pair-bond is a particularly trying evolutionary puzzle that evoked several competing hypotheses (Lovejoy, 1981; Strassmann, 1981; Marlowe, 2003; Hawkes, 2004; Quinlan and Quinlan, 2007; Chapais, 2008). The lack of detailed knowledge on the social organization (solitary, pair-living, group-living), mating system (monogamy, polyandry, polygyny, polygynandry/promiscuous), and social structure (pattern of social interactions and resulting relationships among group members) of various societies complicates the revelation of the evolutionary origins along the phylogenetic lineages throughout the animal kingdom (see Kappeler and van Schaik 2002 for terminology). Inconsistent terminology complicates matters even further. In that respect, the human pair-bond describes the social structure, rather than organization or mating system, of two members of the opposites sex, showing high affiliation but not necessarily living in pairs or being monogamous (Chapais, 2013). In lack of behavioral data on our ancestors, non-human primates state the best for comparison and derivation of human behavioral patterns, mating systems and social styles (Strassmann, 1981; Chapais, 2008; Coxworth et al., 2015).

In birds, about $91 \%$ of species feature at least serial pair-living, though in some species multiple pairs also aggregate in large colonies (Greenwood, 1980). Most mammalian species are classified as solitary (68\%), while far less live in social groups (23\%) or even pairs (9\%) (Lukas and Clutton-Brock, 2013). Mate desertion is thought to be favored by selection because of the limited potential for direct male care during internal gestation and lactation, which are the hallmark features of mammalian life history (Clutton-Brock, 1991). Primates, on the other hand deviate from this general pattern, as $29 \%$ of species are pair-living (Lukas and CluttonBrock, 2013) and over two-thirds of genera (out of 56) show permanent male-female association in stable groups throughout the year (van Schaik and Kappeler, 1997). Moreover, primates have unusually large brains compared to body size and while in primates neocortex size is correlated with group size (Dunbar and Shultz, 2007), in other mammals this relationship does not hold. In carnivores, artiodactyls, and bats pair-living species have significantly larger brain sizes than species from other social organizations (Dunbar and Shultz, 2007). The social brain hypothesis states that cognitive demands increase with group size, and as a consequence the evolution of larger brains is favored in larger groups (Dunbar, 1992; Kudo and Dunbar, 2001). However, social complexity is not necessarily restricted to large groups, but more to the social interactions needed to synchronize behavior. For example, large 
ungulate aggregations where individuals hardly interact with each other (Parrish et al., 1997) are cognitively less demanding for individual group members than highly synchronized pairs (Dunbar and Shultz, 2007). The explanation why in primates brain size is correlated with group size is that group-living primates develop 'bondedness' to several group members (Wrangham, 1980; Smuts, 1985; Sterck et al., 1997; Bergman and Beehner, 2015) that are individually comparable to a pair-living partner (Dunbar and Shultz, 2007).

A recently emerging debate between Lukas and Clutton-Brock (2013) and Opie, Atkinson, Dunbar and Shultz (2013) about the evolution of pair-living or social monogamy underlines the importance to fully understand how and why primate societies generally deviate from mammals. Both groups defined a solitary lifestyle as ancestral origin but differed in their modeling to reach social monogamy. In mammals, social monogamy is thought to have evolved directly from a solitary ancestor (i.e. territorial females and free ranging males) as a result of female competition, female intolerance of same-sex conspecifics, low female density and male's inability to monopolize access to several female territories (Lukas and CluttonBrock, 2013). In primates however, more or less stable social groups are thought to have evolved from aggregating solitary ancestors for anti-predator benefits (Shultz et al., 2011), and infanticide drove the evolution of socially monogamous pairs (Opie et al., 2013). A lively debate followed. Lukas and Clutton-Brock argued that bias in Opie et al's data (in terms of report for infanticide in captive or laboratory studies) led to a misinterpretation of results. This emphasizes the need for studies on wild populations that represent the natural behavior of a species (Lukas and Clutton-Brock 2014; but see Opie et al. 2014 for a reply). Interestingly, Lukas and Clutton-Brock (2013) did not mention how primate year-round multimalemultifemale groups fit into the sequence of evolution of social monogamy in mammals, let alone the evolution of human pair-bonds in a group-living organization with pronounced and very differentiated social structure. This is surprising, as human pair-bonds are considered to have evolved from large multi-male systems (Chapais, 2008).

Three mutually non-exclusive hypotheses about the evolution of the human pair-bond are currently suggested. The paternal-care hypothesis (Lovejoy, 1981; Kaplan et al., 2000; Marlowe, 2003; Quinlan and Quinlan, 2007, 2008) assumes that paternal investment was crucial during some point of female lactation and gestation to ensure offspring survival. The male mate-guarding hypothesis (Hawkes, 2004; Chapais, 2008; Coxworth et al., 2015) states that males were forced to join into elaborated mate-guarding for securing mating during the receptive phase of females and fending off competitors in times of male-biased sex ratio and increased male competition. The infanticide hypothesis (Palombit, 1999; Hawkes, 2004) suggests that males stay with females to defend their offspring against conspecific males.

Interestingly, similar mutually non-exclusive hypotheses were suggested for the evolution of male-female affiliative relationships in non-human primate multimale-multifemale groups. Females and her offspring may receive protection from affiliated males against 
potentially infanticidal males (Palombit et al., 1997; van Schaik and Kappeler, 1997; Paul et al., 2000; Engh et al., 2006; Palombit, 2009) or non-lethal harassment by conspecifics (Busse and Hamilton, 1981; Nguyen et al., 2009). The affiliated male may also become a future caretaker for the female's offspring (Seyfarth, 1978a). Males on the other hand may enhance mating (Smuts, 1985) or paternity (Ostner et al., 2013) success with the affiliated female, gain access to her offspring to use it as social passport (Deag, 1980), or providing paternal care for their own biological offspring (Taub, 1980; Charpentier et al., 2008; Huchard et al., 2013; Ostner et al., 2013).

My study set out to elucidate the functional significance of male-female affiliative relationships or 'friendships' (Smuts, 1985; Silk, 2002) in non-human primate multi-male groups and their implications for the evolutionary origin of the human pair-bond. In particular, my study provides valuable findings on the nature of opposite-sex relationships in large groups with promiscuous mating systems, in terms of quality, stability, and resulting costs and benefits for females in wild Assamese macaques (Macaca assamensis). In the following introduction, I will review empirical evidence on the formation of affiliative relationships between males and females with the emphasis on multimale-multifemale groups. Further, I will discuss paternal care with its definitions and implications. I will stress the fact how little we know about female competition for males, particularly outside the mating context, and why that may be the case. I will further discuss the evolution of pair-bonds or social monogamy in primates in general and in humans in particular and emphasize the role concealed ovulation may have played. Last, I will introduce the study species Assamese macaques in Phu Khieo Wildlife Sanctuary, Thailand, as well as the main goals of my study. 


\section{OPPOSITE-SEX SOCIAL BONDS IN MULTI-MALE PRIMATE GROUPS}

Primates most commonly form female-bonded groups, where females are the philopatric sex, males emigrate from natal groups, and females form strong and stable socio-positive relationships, or social bonds, to each other (Wrangham, 1980). These female-bonded groups may have evolved in response to intra-group competition for monopolizable resources (Wrangham, 1980; Sterck et al., 1997). Here, kin selection theory suggests that high relatedness between group members could offset costs associated with group-living and promote cooperative behavior (Hamilton, 1964).

The presence of several adult males may be beneficial for females in terms of enhanced competitive ability of the group in inter-group interactions (Wrangham, 1980), or as protectors from potentially infanticidal males (Palombit et al., 1997; Borries et al., 1999a). However, males also incur potential costs for females. In species where resource holding potential (RHP; based on sexual dimorphism and dominance) of males is higher than of females (Plavcan, 2001), males can inflict more severe injuries during agonistic interactions than females do (Smuts, 1985; Chapais, 1986), particularly in the context of sexual coercion (MacCormick et al., 2012), and gain higher priority of access to food (Rose, 1994). Additionally, male group members may act infanticidal, when paternity can be ruled out and reproductive benefits can be gained (Borries et al., 1999b).

In females, counterstrategies may have evolved to balance costs and benefits from living with multiple males. In primates, the evolution of exaggerated sexual swellings seems to be connected with promiscuous mating systems in multi-male groups (Nunn, 1999). In this respect, sexual swellings may either increase paternity certainty (if probability of ovulation correlates with swelling size), or confuse paternity certainty (if ovulation does not occur precisely at peak swelling), depending on what serves the female best (Nunn, 1999). Paternity confusion may diminish infanticide from inside the group (as each male might be the father), while paternity certainty might increase the male's investment in infant protection. Alberts and Fitzpatrick (2012) proposed the 'Paternal Care Hypothesis' to explain the function of sexual swellings as a female tool to elicit the right quantity and quality of male care for her offspring. They predicted that true paternal care (i.e. males accurately differentiate and provide assistance to biological offspring) would be most common in species with exaggerated sexual swellings that reliably signal ovulation and where males can successfully monopolize mating with a female at the time of ovulation (Alberts and Fitzpatrick, 2012). Those mechanisms enhance paternity certainty and may in turn increase paternal investment. Slow life history of primates (i.e. long lives and low fertility) may select for males that invest in the few offspring they produced during their lifetime (Alberts and Fitzpatrick, 2012). When paternity is less certain, males may have to balance costs resulting from care for non-related immatures and 
costs that may result from denying care to potential biological offspring (Moscovice et al., 2009).

Another female strategy to relax tension with males in the group may be the formation of affiliative relationships (Smuts, 1985). Byrne, Whiten and Henzi (1989) argued that all baboons are cross-sex bonded, meaning that social relationships to both sexes matter, instead of only female-female relationships as implicated by the terminology of 'female-bonded' groups (Wrangham, 1980). Accordingly, social connectedness in yellow baboons (Papio cynocephalus) to both females and males enhances female longevity (Archie et al., 2014). Females might focus their attention more towards males or females according to the need for coalitionary support, which in turn might create a very flexible social system between the formation of multi-male and one-male groups (Barton et al., 1996). Ecological and social conditions may influence the form of bonding expressed in different species. Hence, groups may be held together not only by the social bonds formed within the philopatric sex, but also by opposite-sex bonds, or both (Byrne et al., 1989). Byrne et al.'s (1989) theory may not only be applicable to baboons, but to any species with a flexible social structure undergoing ecological variations. Differentiated relationships between males and females may have much more importance in those terms than formerly suggested.

The study of social relationships between males and females in multimale-multifemale primate groups has a long history. In primatology, bonds between adults may be best described as predictable relationships that can be assessed by rates of affiliative interaction, proximity scores and a measure of reciprocity between two individuals (Fuentes, 2002). Most extensively studied are baboons and macaques, but there are also studies from lemurs, capuchins and recently chimpanzees (see Chapter 1 ). In the following section, I will describe how different species form opposite-sex relationships, in terms of intensity or differentiation, stability and equitability, which are the hallmark features to define a social bond between two individuals (Ostner and Schülke, 2014). The pure description of social relationships, irrespective of statistical testing of hypotheses, may further help to understand the differences between species, genera, or families. I will focus on those species that build multimalemultifemale groups and promiscuous mating systems, where both males and females can build differentiated relationships to several individuals. For convenience, I will use the term 'friendship' to describe an affiliative relationship between two individuals that is clearly differentiated from other dyadic relationships in terms of time spent together and amount of grooming, without necessarily assuming any emotional bond between two individuals, as implied in human friendships. As most studies use different measures and scores to define 'friends' the term does not necessarily have the same meaning. I will thus provide descriptive data when possible. 


\section{Baboons}

A number of studies have examined opposite-sex friendships in baboons. All baboon females exhibit large sexual swellings that indicate relatively reliable female ovulation (Nunn, 1999) in an aseasonal breeding system. In olive baboons (Papio anubis) male knowledge of a female's actual fertile phase seems to be based on close association in mate-guarding behavior (Higham et al., 2009), while in chacma baboons (Papio ursinus) long-term residents seem to track female fertile phases and time mate-guarding accordingly (Weingrill et al., 2003). In yellow baboons (Papio cynocephalus), sexual swellings are a reliable signal for conception probability within and among cycles and males adjust their behavior accordingly, with the alpha male gaining the most access to receptive females (Gesquiere et al., 2007). Almost all females show a strong preference for one preferred male grooming partner, with whom they also spend the most time, during some stages of their reproductive cycle (Smuts, 1985; Palombit et al., 1997; Lemasson et al., 2008; Nguyen et al., 2009). While in olive baboons, friendships seem to be consistent over time (Smuts, 1985), in chacma and yellow baboons male-female friendships are basically linked to female reproduction and likelihood of paternity (Palombit et al., 1997; Moscovice et al., 2010; Baniel et al., 2016). Bonding in time of gestation and lactation may be constant (Nguyen et al., 2009; Baniel et al., 2016).

With the exception of Kinda baboons (Papio kindae; Weyher et al. 2014), females are more likely to groom a males than vice versa (Palombit et al., 1997; Nguyen et al., 2009; Baniel et al., 2016), while the maintenance of close proximity can be associated with both sexes (Smuts, 1985). Reports of infanticide vary across species, with chacma baboons featuring rather high rates (37\% of infant deaths connected to infanticide: Palombit et al. 1997), olive baboons intermediate (ambiguously reported as high by Lemasson et al. 2008 or low by Smuts 1985) and low rates in yellow baboons (Nguyen et al., 2009). Protection against infanticidal males may be the best explanation for the formation of male-female friendships in chacma (Palombit et al., 1997), but not in olive and yellow baboons. However, friendships are overall connected to male infant handling and there is strong evidence that males may serve females and their offspring (Smuts, 1985; Lemasson et al., 2008; Moscovice et al., 2009; Nguyen et al., 2009; Huchard et al., 2013), or the biological offspring only (Buchan et al., 2003) as protectors against non-lethal aggression by group members. Number of male friends or social connectedness correlate with female dominance rank, indicating potential female competition for access to male partners in olive (Lemasson et al., 2008), chacma (Palombit et al., 2001; Baniel et al., 2016) and yellow baboons (Archie et al., 2014). 


\section{Macaques}

Macaques vary both in the seasonality of breeding and exhibition of sexual signals (Nunn, 1999). Only few macaques (e.g. crested macaques, Macaca nigra, moderately seasonal Kerhoas et al. 2014) are not seasonal breeders, while on the other hand only few do not exhibit reliable sexual signals to indicate ovulation (e.g. toque macaque, $M$. sinica). Barbary $(M$. sylvanus), Japanese (M. fuscata) and crested macaques exhibit large sexual swellings (Nunn, 1999; Heistermann et al., 2005). Swelling size, shape or color are reliable indicators for female ovulation so that males can pinpoint mating behavior accordingly (Brauch et al., 2007; Higham et al., 2012). Rhesus macaques ( $M$. mulatta) do not signal fertility by sexual swellings. Instead, coloration of skin indicates intra-cycle probability of fertility, while changes in the facial skin coloration contains information about the precise timing for males (Dubuc et al., 2009). Relationships with the opposite sex seem to be rather undifferentiated and restricted to mating season in Barbary macaques (Small, 1990a), when males invest more into the maintenance of close proximity and grooming with females than vice versa and grooming episodes are extremely short due to female retraction. In Japanese and rhesus macaques, some malefemale dyads may form friendships based on grooming and time spent in close proximity that are stable over time (Takahata, 1982; Manson, 1994; Majolo et al., 2010). Differentiated relationships may also be found in crested (Kerhoas et al., 2016) and toque macaques (Ratnayeke, 1994). There is no evidence that friendships may influence female mate choice (Manson, 1994), but friendship increases the likelihood for paternity in rhesus macaques (Kulik et al., 2011).

As in baboons, females invest more in grooming than males (Takahata, 1982; Chapais, 1983a; Tsukahara, 1990; Ratnayeke, 1994; Reed et al., 1997; Majolo et al., 2010), whereas males may be more responsible for maintaining spatial proximity (Manson, 1994). Generally, high ranking males may be more attractive for females (Takahata, 1982; Hill, 1990), and in rhesus macaques the alpha female also seems to be more attractive for males (Chapais, 1983a). Both males and females aid each other in agonistic encounters (Takahata, 1982; Chapais, 1983b; Small, 1990a), particularly in terms of male aid towards female friends (Hill, 1990; Manson, 1994). Females may increase their dependent rank in the male's presence in the context of feeding (Takahata, 1982) or just be tolerated more by the befriended male (Hill, 1990). Males interact frequently in a friendly way with infants. This infant-handling behavior is not related to paternity in Barbary and crested macaques (Kuester and Paul, 1986; Paul et al., 1996; Ménard et al., 2001; Kerhoas et al., 2016), but to the former affiliative relationship to the female in Japanese macaques (Takahata, 1982). Reports on infanticide are rare, except for crested macaques (Kerhoas et al., 2014), where alpha male tenure is comparably short (Marty et al., 2015) and infants seem to seek males' vicinity, which may be triggered by mothers' behavioral attachment to the male (Kerhoas et al., 2016). Only rhesus macaques may reliably discriminate biological offspring (Langos et al., 2013), yet they do not support their offspring in 
agonistic conflicts (Kulik et al., 2011). There is evidence in Japanese, toque and rhesus macaques that females may compete for access to male partners (Takahata, 1982; Manson, 1994; Ratnayeke, 1994).

\section{Chimpanzees}

In contrast to baboons and macaques, chimpanzees (Pan troglodytes) form male-bonded groups with female dispersal. Because females usually immigrate only once into a new group, female tenure is extremely long (Goodall 1986; see for citation Machanda et al. 2013). Females exhibit large sexual swellings that seem to be a reliable indicator of ovulation for males (Deschner et al., 2004) with aseasonal breeding system. Differentiation of relationships based on association patterns and grooming is very pronounced, and only for a fraction of malefemale dyads reliable indices can be calculated for grooming alone (Machanda et al., 2013). Male-female association patterns are highly influenced by female estrus, as is the direction of grooming. Females in estrus are found more frequently in parties with males than anestrus females, and while male-anestrus-female grooming direction is almost symmetric, estrus females are much more groomed by males than vice versa (Machanda et al., 2013). Nevertheless, association patterns may be stable over years (Langergraber et al., 2013). Particularly newly immigrated females that receive a high degree of aggression from resident females may benefit from associating with males in general by gaining support against conspecific harassment (Kahlenberg et al., 2008). Unfortunately, no studies on dyadic effects are available so far. Infanticide in chimpanzees seems to be rather common, performed by both males and females (Arcadi and Wrangham, 1999). Although males are able to distinguish biological offspring from others, they do not provide any obvious paternal care except reduced rates of aggression towards biological offspring (Lehmann et al., 2006).

\section{Humans}

In the former sections, I described a social bond, an affiliative relationship, or a friendship as neutral differentiation of spatial and social interaction between two individuals. Describing human relationships does not completely allow for this kind of objectivity any longer, because human relationships are invariably entangled with emotions and much data survey is based on questionnaires. The description of human relationships is further complicated by cultural influences on human societies, particularly when enforced marriage plays a role (Fletcher et al., 2015). I will provide a short review on different kinds of social structure and mating systems in human societies, trying to describe humans as just another primate species that they are (Daly and Wilson, 1999).

Humans are aseasonal breeders with females undergoing a menstrual cycle and ovulation about once a month, if not lactating or pregnant (Howie and McNeilly, 1982), and exhibit copulations constantly across the female cycle except during the five days of menses 
(Brewis and Meyer, 2004). Those traits present a contrast to any non-human primate. Female ovulation in humans may be physically entirely concealed, which is rare among primates. Though Hazda men (mobile hunter-gatherers in Tanzania) apparently correctly associate mating with pregnancy, they incorrectly assess the probable timing of ovulation right after the menses (Marlowe, 2004). Behaviorally on the other hand, females indicate ovulation by promoting their appearance and they are generally perceived differently and more sexually attractive by males during the peak of fertility (Roberts et al., 2004; Haselton et al., 2007; Gangestad and Thornhill, 2008; Fink et al., 2012). To establish a mating system in humans is far more difficult than in non-human primates. Sociosexuality (i.e. a self-assessed measure of individual differences in mating strategies) varies widely both between sexes and cultures with generally higher male than female tendency to promiscuous mating (Schmitt, 2005). However, in contrast to non-human primates, mating behavior in humans may not conform to a single universal pattern (Brown et al., 2009). Monogamy is only strictly enforced in $17 \%$ of human societies, while in $80 \%$ there is a mixed system of monogamy and polygyny (reviewed by Chapais 2013). The human pair-bond (i.e. a long-term affiliation including a sexual relationship; Quinlan and Quinlan 2007) is one of the main distinctive features of human social structure (Chapais, 2013), with almost all human societies featuring pair-bonds (Coxworth et al., 2015). Human groups are composed of multifamily groups that are large multimale-multifemale groups with stable pair-bonds between adult males and females that may or may not be monogamous (Chapais, 2013). While in non-human primates social structure is set to some degree, in humans the definition of social structure is complicated by a large variation of cultural differences between populations (Chapais, 2013). The stability and steadiness of permanent pair-bonds (or marriage) leads to enhanced longevity in both sexes (Friedman et al., 1995), indicating the significance of pair-bonding in humans. Female competition for male partners is rather common and may involve physiological costs (Fawcett and Johnstone, 2003; Campbell, 2004; Stockley and Campbell, 2013). Killing of immatures is not rare and may be performed, in the case of male infanticide, by both, the biological father or the mother's new mate (Daly and Wilson, 1994). Although paternal care in humans may range from hardly any physical contact between males and babies to extensive carrying and provisioning (Fernández-Duque et al., 2009), the evolution of permanent pair-bonds out of multimalemultifemale groups in humans is often considered to be tightly linked to paternal care (Alexander and Noonan, 1979; Lovejoy, 1981; Kaplan et al., 2000; Quinlan and Quinlan, 2007). 


\section{PATERNAL CARE}

'Paternal care' is a behavior performed by a putative or social father that appears to have a positive effect on infant development, growth, well-being, and/or its survival (Fernández-Duque et al., 2009). By this definition, there is no implication of intentional actions on the male's side, nor are actual costs a necessary condition. Rather, paternal behavior can just be a by-product of male behavior. Still, only behaviors count as paternal care that would not be carried out in the absence of immatures. In that respect, a male's presence may lower predation risk for the group and hence also the immature's, but the male would serve this function regardless of immatures' presence at the time (Woodroffe and Vincent, 1994). 'Paternal investment' on the other hand is a behavior performed by a putative or social father that increases the probability of the infant's survival (Fernández-Duque et al., 2009). Here, males may suffer some costs to increase the chances of the immature's survival, and accordingly males should adjust their investment to balance involved costs and benefits. Noteworthy is that in both definitions the male can be the biological or behavioral father of the offspring. In contrast, 'true paternal care' implicates that males have to be able to distinguish between biological offspring and others, and adjust their behavior accordingly (Alberts and Fitzpatrick, 2012). Here, I will use the term 'paternal care' for all male care performed in the above mentioned sense, with no regards to actual genetic relations between the involved individuals. I will use 'true paternal care' only in situations when males actually differentiate between immatures based on genetic relationships.

The broad definition of paternal care allows a huge variation of behaviors contributing to the offspring's fitness. Paternal care may have a direct physical impact on infants, in terms of thermoregulation or huddling, provisioning or food sharing, carrying, cleaning or grooming, socialization, or agonistic support (Kleiman and Malcolm, 1981; Woodroffe and Vincent, 1994). Additionally, males and immatures may bond during infancy and immatures may receive further attention through the post-weaning phase. The latter is only applicable when males and immatures experience a prolonged period of mutual interaction. While some male behaviors may involve little costs (e.g. huddling, grooming, socialization), others may be quite costly (like agonistic support). Males should adjust costly behavior according to the benefits they receive through the action and invest more in their own biological offspring than in unrelated individuals. On the other hand the 'female choice' hypothesis assumes that male care for infants may be a mating strategy that increases the chances for copulations with the mother in the future (Smuts and Gubernick, 1992; van Schaik and Paul, 1996) or with additional females that observe the performed behavior (Seyfarth, 1978a). If immature benefits are high and male costs low, males may better provide paternal care for unrelated immatures than miss the chance to provide it for biological offspring (Moscovice et al., 2009). 
Paternal care is widespread among birds and fish, but rare in mammals (Kleiman and Malcolm, 1981; Fernández-Duque et al., 2009). Within mammals, primates again build an exception along with carnivores and rodents (Kleiman and Malcolm, 1981; Woodroffe and Vincent, 1994). Although it was long considered that paternal care was the offset for the development of pair-living and monogamy in primates (van Schaik and Dunbar, 1990), phylogenetic evidence suggests that paternal care may be rather a consequence than a cause of monogamy (Lukas and Clutton-Brock, 2013). Among all pair-living, monogamous hylobatids, the siamang (Symphalangus syndactylus) is the only species that provides paternal care (Fernández-Duque et al., 2009). Not the mating system or social organization but rather the benefits received by providing help raising offspring may be the better explanation for the evolution of extensive paternal care (Wright, 1990). Researchers long neglected the possible importance of paternal care in group-living species with promiscuous mating systems. Recent evidence indicates that males may contribute more to immature fitness than previously assumed (Palombit et al., 1997; Buchan et al., 2003; Charpentier et al., 2008; Moscovice et al., 2009; Huchard et al., 2013).

In baboons, males protect immatures from agonism through conspecifics, either from lethal aggression in terms of infanticide (Palombit et al., 1997) or from non-lethal aggression (Buchan et al., 2003). Male yellow baboons may be able to distinguish biological offspring from others, and accordingly, females' male friends that are not the sires, do not act as behavioral fathers (Buchan et al., 2003). In this species, a differentiation between true paternal care and male care that passively comes along is evident. The male only provides costly active agonistic support towards those immatures that are clearly his own offspring. However, they may provide passive care in terms of tolerating a mother and her offspring close by, shielding them from conspecific aggression (Nguyen et al., 2009). Both chacma and olive baboons do not seem to differentiate between biological offspring and others in a reliable way. As infanticide can be high in both species, males may have much more to lose when denying protection to an unrelated immature than falsely denying protection to their own biological offspring (Moscovice et al., 2009). Male Barbary macaques show excessive male infant handling. How beneficial this behavior may be for immatures, is under debate (Kuester and Paul, 1986). In line with this argument, neither paternity nor mating history play a role in males' infant choice (Ménard et al., 2001). Female friend's dependent offspring may seek out males in Japanese and crested macaques, independent of paternal effects (Takahata, 1982; Kerhoas et al., 2016). While there is no evidence for agonistic support, the mere presence may be beneficial for the infant in terms of protection or socialization. In both species, paternity does not seem to play a major role for male-infant bonding, though chances for paternity certainty are relatively high. However, the need to differentiate between biological and non-offspring is not as high, if males do not invest as much. Rhesus males on the other hand seem to differentiate between kin and non-kin by higher affiliation (Langos et al., 2013). It was suggested, that female rhesus 
macaques discriminate not only maternal but even paternal kin (Widdig et al., 2001). Here, male-immature affiliation behavior may have a beneficial effect particularly for female offspring and mitigate later paternal kin discrimination. Overall, in non-human primates males often associate with immatures and provide some paternal care that varies widely between species.

In human societies, the expression of paternal care varies from absence to direct care (Fernández-Duque et al., 2009). A sign of direct paternal care in humans is holding time of the infant (Marlowe, 1999), ranging in foraging societies from fathers that rarely interact with infants or young children to those that hold young infants up to $22 \%$ of their time. Intercultural variability in both developed and developing countries may be associated to local ecology and social environment (Fernández-Duque et al., 2009). Variation in degrees of paternal care in humans has been connected to household compositions, mating opportunities and resources, extrinsic morality and pathogen stress (Hewlett, 1991; Marlowe, 1999; Quinlan, 2007). Even within societies that are known for a high degree of paternal care significant variation among men can be found (Hewlett, 1991). Provisioning of offspring with prolonged dependency as in humans is of utmost importance to enhance offspring development (Lovejoy, 1981; Kennedy, 2005). In line with this argument, male Hazda hunters provide food to females and offspring in critical periods where female independent provision ceases (Marlowe, 2003). In societies where infanticide and abuse are common, the presence of protective biological fathers increases the offspring's chance of survival (Quinlan and Quinlan, 2007). Evidence of how important fathers are for the survival of their offspring under less intense conditions is mixed (Sear and Mace, 2008; Winking et al., 2011).

Paternal care usually has a positive impact on immature's fitness, regardless of how minor male contribution may appear. Particularly in animals with slow life histories, like primates, offspring survival accounts for much of the variation in female lifetime reproductive success (Clutton-Brock, 1988). Accordingly, males providing paternal care are particularly likely to attract female competition (Palombit et al., 2001). 


\section{FEMALE COMPETITION}

Sexual selection theory predicts that intrasexual competition for mates is generally higher in the sex with the lower investment in offspring (Trivers, 1972). However, evidence of female competition for mates is accumulating, even in social systems where males provide no or little care for the offspring and female investment is high (Stockley and Bro-Jørgensen, 2011). The best explanation for female competition for males not only as mating partners may be that males provide other resources, apart from sperm, for females or their offspring. Stockley and Bro-Jørgensen (2011, p. 350) stated, "if males vary in the quantity or quality of resources they provide, females may compete for males as indirect manifestation of resource competition". Thus, we can extend this argument from female competition for mates to competition for male friends if those provide valuable benefits for female reproductive success. Besides paternal care, a high dominance rank of a male partner may be of special interest for females through improved priority of access to resources and male's ability to protect the female against conspecifics (see details in Chapter 3). Although the study of female competition for partners outside of the mating context is well developed in humans, there is lack of evidence regarding mammals and non-humans primates in particular.

The presence of female competition may be underestimated, because it is less conspicuous than male competition for mates (Bro-Jørgensen, 2002; Clutton-Brock and Huchard, 2013a). In mammals, female investment in offspring is generally higher, potentially leading to females avoiding physical aggression, particularly in context with dependent offspring (Huchard and Cowlishaw, 2011; Campbell, 2013). Additionally, kin selection theory assumes that competition should be less intense in female-bonded primate groups (Hamilton, 1964; Wrangham, 1980). In species with linear dominance hierarchies females may settle competitive interactions by silent supplants, rather than escalated fighting, because the threat of aggression is sufficient to settle the matter (Stockley and Campbell, 2013). In line with this argument, female chacma baboons achieve access to high-ranking males according to dominance rank and lower-ranking females are replaced from existing friendships (Palombit et al., 2001). Alternative costs of competition may be increased stress among competitors, particularly in the case of failure, which in turn may be potentially harmful (Faer et al., 2005; Salmon et al., 2008). In chimpanzees, with male-bonded group structure and female dispersal, female aggression is potentially harmful and particularly directed at newly immigrating females that post a threat of future resource competition (Kahlenberg et al., 2008; Lehmann and Boesch, 2009). Social bonds between female chimpanzees reduce the rate of agonistic interactions (Lehmann and Boesch, 2009). The same mechanism is assumed to have shaped female friendship and cooperation in humans (Stockley and Campbell, 2013).

In humans, intrasexual competition for partners is the driving factor for female competition for status, general competitiveness, perfectionism, body expectations, drive for 
thinness, bulimia and anorexia (Faer et al., 2005). Women usually compete with respect to physical traits that may be desired by mates (Fink et al., 2014). While women may be more selective than males regarding their mating decisions, they still need to compete for male commitment (Faer et al., 2005). When physical traits fail, competitive interactions in humans are mostly based on threats or indirect aggression and may be so subtle to go unnoticed by observers (Benenson, 2013; Campbell, 2013; Cant and Young, 2013; Vaillancourt, 2013). Women often use the threat of social exclusion as punishment for individuals who fail to adjust their behavior to a covert threat, which may be a very effective form of punishment as it only involves minimal costs for the actor (Benenson, 2013; Campbell, 2013; Vaillancourt, 2013). This kind of punishment can have extreme effects on the recipient, because of the strong friendships formed between individuals and the emotional interdependence connected with it, leading to depression or even suicide (Benenson, 2013). However, also in animals the threat of social exclusion may involve significant costs for females (Clutton-Brock and Parker, 1995).

When competition for partners is costly, individuals may differ in their preferences. For poor competitors it may be a better strategy to target low-quality partners and hence avoid costly competition (Fawcett and Johnstone, 2003). Differences in competitive abilities result in assortative bonding with respect to the partner's quality, which further is enhanced by the variation in individual preferences (Seyfarth, 1977; Fawcett and Johnstone, 2003). In a hypothetical scenario where females alone decide on the outcome of dyadic relationships, assortative bonding might work out as follows: When males with high dominance rank are the most attractive to females, females should 'cue' for the highest-ranking male according to their dominance rank. Furthermore, when males that provide paternal care are independently attractive, the highest-ranking female may choose between those traits. Consequently, the linear relationship of assortative bonding depends on 1) the number of traits a female may choose from, 2) how those traits are interrelated, and 3) their significance - apart from the so far ignored male partner choice. Female competition for males may be underappreciated in literature (but see Stockley and Bro-Jørgensen 2011; Clutton-Brock and Huchard 2013b for female competition for mates), however careful reading reveals that female access to males is often based on dominance rank (see above; olive, chacma and yellow baboons, toque, rhesus and Japanese macaques), indicating female competition for bonding partners. 


\section{EVOLUTION OF PERMANENT PAIR-BONDS IN PRIMATES}

At present, humans may be the most successful species and the pair-bond is supposed to be the core feature of human evolutionary success (Chapais, 2008, 2013). Why do most nonhuman primates not form permanent pair-bonds similar to those in human societies? Either, changes in social organization and mating system are not possible for reasons of genetic manifestation or not suitable because changes would not improve reproductive fitness under the current ecological conditions (Barton et al., 1996). For example, chacma baboons have the disposition for transition between the typical multimale-multifemale condition (featured by savanna baboons) and one-male units, dependent on ecological conditions like food availability and predation risk (Barton et al., 1996). Hamadryas baboons, on the other hand, may be genetically set to a degree that they have lost the social flexibility of transition to multimale-multifemale groups, even when ecological conditions would favor a different system (Barton et al., 1996). Interestingly, hamadryas baboons with their strict one-male unit multilevel society and polygynous mating system often serve for evolutionary theories on the human pairbond (Swedell and Plummer, 2012) since human societies are supposed to resemble their system (Chapais, 2013).

However, other studies argue that the evolution of monogamy in primates is much more likely to have originated in large multimale-multifemale groups (Alexander and Noonan, 1979; Shultz et al., 2011). Shultz and colleagues (2011) found that the reversible-jump model fitted best the data of the evolution of monogamy in primates (see Figure 0.3). The reversible-jump model states that multimale-multifemale groups developed from a solitary lifestyle, while pairliving, as well as one-male groups, evolved out of multimale-multifemale groups. Only transition between one-male groups and multimale-multifemale groups would still be possible. Once the solitary lifestyle was discarded, the model does not allow for return. Also pair-living is considered to be a dead end without any chances to return back to group-living (Shultz et al., 2011). As already mentioned above, a debate followed two years later, because the evolution of monogamy in primates does not seem to fit into the mammalian model (Lukas and Clutton-Brock, 2013; Opie et al., 2013). The most vigorously debated point was whether the risk of infanticide did promote the evolution of monogamy or not, with both groups of authors finding differing results (Lukas and Clutton-Brock, 2014; Opie et al., 2014). Pair-living in primates may as well have evolved directly from multimale-multifemale groups, even without the risk of infanticide being the main driving selective force. 


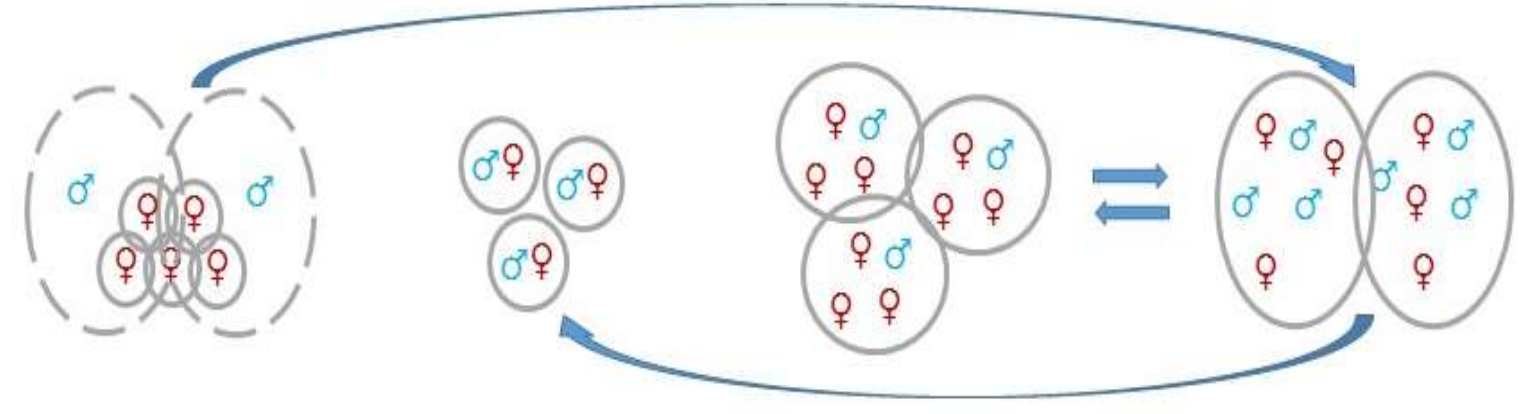

Figure 0.1: The reversible-jump model after Shultz et al. 2011. It shows the one-way transition from solitary lifestyle to group-living, further to monogamy and the two-way transition between one-male and multi-male groups.

Concealed ovulation is suggested to have played a role in the evolution of pair-bonds from multimale-multifemale groups (Strassmann, 1981). The male-male competition hypothesis suggests that concealed ovulation lowers competition between males, because the operational sex-ratio (ratio of fertile individuals at a given time) is confounded when fertility of females is harder for males to assess (Andelman, 1987; Marlowe and Berbesque, 2012). Reduced male-male competition, may lower harassment to females and her offspring (MacCormick et al., 2012), lowering the risk for infanticide (Hrdy, 1979) or increase male participation in intergroup encounters (Wrangham, 1980). The male-parental-care hypothesis states that females conceal ovulation to enhance paternal investment in offspring (Alexander and Noonan, 1979). According to the pair-bond hypothesis females enforce a monogamous mating system on males by minimizing external indicators for fertility while prolonging the period of sexual receptivity (Morris, 1967). Hereby, it is implicated that, from a female's perspective, a monogamous mating system would be preferential to a polygynous one, because of the female potential for monopolization of male attendance. When exactly concealed ovulation evolved in early hominins is hard to say as such physical traits do not fossilize (Andelman, 1987).

If concealed ovulation evolved prior to the pair-bond in humans, it may have set in motion a cascade of events that enforced pair-bonding (Strassmann, 1981). Here, the reduction of male monopolization potential of fertile females would have lowered reproductive success of polygynist males. Instead, males providing paternal care may have enhanced own reproductive success and that of female mates, leading to females choosing paternal males as a partner. Evolution would in turn favor paternal behavior in males, if paternal males sire more offspring, which would further enforce permanent pair-bonds (Strassmann, 1981). To shed light on the evolution of permanent pair-bonds from multimale-multifemale groups, we may best study species that feature concealed ovulation, potential for paternal care, and indication for opposite-sex pair-bonds. 


\section{STUDY SPECIES}

Assamese macaques represent a suitable study species to assess the adaptive significance of male-female relationships and draw conclusions on the evolutionary origin of the human pair-bond. The species differs from general models in several ways and may resemble the human system more than most other species. Here, I will introduce the study population at Phu Khieo Wildlife Sanctuary, Thailand (see details on the study site in Chapter 1).

Assamese macaques belong to the sinica species group within the macaques, along with toque ( $M$. sinica), bonnet $(M$. radiata), and Tibetan macaques ( $M$. thibetana) (Thierry et al., 2000). Interestingly, the lineage does not fit into the general connection of exaggerated sexual swellings and multi-male grouping as none of the species develops sexual swellings (Nunn, 1999). Assamese macaques live in groups comprising multiple adult males (6-12 in this study) and females (10-15 in this study) with a large number of immatures (24-34 in this study). The species is highly arboreal and features a mostly frugivorous feeding ecology with $40 \%$ of their active time spent feeding (Schülke et al., 2011).

Females build strong and differentiated affiliative relationships with group members of the same sex (Macdonald, 2014). There is no evidence for grooming related agonistic support (Macdonald et al., 2013), or increased energy intake due to dominance rank (Heesen et al., $2013,2014)$. Rather, females employ alternative mechanisms to avoid aggressive competition for food and in this context strong social bonds to other females enhance feeding tolerance (Heesen et al., 2014). The importance of access to food resources is mirrored in the result that an increase in food availability has a positive effect on both female energy intake and conception rates (Heesen et al., 2013). Reproduction in the study population is highly seasonal, with a mating season from October to January and a birth season from April to June (Fürtbauer et al., 2010). On average, females give birth every one or two years, with annual birth linked to early parturition in the preceding birth season (Fürtbauer et al., 2010). Females are highly effective in concealing ovulation from males (Fürtbauer et al., 2011a), exhibit highly synchronous receptive phases (Fürtbauer et al., 2011b), and hence lowering male monopolization potential. Consequently, they achieve to mate with all males present in the group, leading to a shallow mating and paternity distribution with a proportion of $29 \%$ respectively for the dominant male (Sukmak et al., 2014). Males form strong social bonds (Kalbitz et al., 2016) that enhance coalitionary support and ultimately increase the rank in the male dominance hierarchy (Schülke et al., 2010).

Although females mate promiscuously, leading to effectively confused paternity, they bias mating selectively towards specific males irrespective of male dominance rank (Fürtbauer et al., 2011a). Dominant males do not effectively monopolize mating with fertile females, and consortships (i.e. mate-guarding in a sexual context) not necessarily encompass female fertility (Fürtbauer et al., 2011a). Spatial association patterns between males and females are stable 
over at least two to three years (Ostner et al., 2013). Strong associations with a female during the mating season are predictive of male paternity success with that female and followed by male-offspring association before weaning. Additionally, paternity is an independent predictor of male-immature association beyond weaning age, which is an indicator for true paternal care' (Alberts and Fitzpatrick, 2012) in this species (Ostner et al., 2013). Infants form differentiated relationships to males that extend well beyond the highest risk of infanticide (Minge et al., 2016). Immatures are mainly responsible for the maintenance of proximity, receive agonistic support from the male, and in the mother's absence they actively seem to seek the male's protection (Minge et al., 2016).

\section{THESIS AIMS}

My study aims to elucidate the adaptive significance of male-female relationships in multimalemultifemale primate groups, with its implications for the origin of the human pair-bond. By combining behavioral data of three non-consecutive years and two macaque groups, with feeding data and hormonal analyses regarding female glucocorticoid metabolite levels (stress), I will assess the significance of relationships particularly for female Assamese macaques. Assamese macaques pose a promising study species because of their deviation from general non-human primate models, by a physiologically completely concealed ovulation in a highly seasonal and promiscuous mating system, where males feature traits of paternal care. In the following, I will describe the specific aims of each chapter in detail.

Chapter 1 serves to describe the formation of affiliative relationships between male and female Assamese macaques. Here, I will investigate the differentiation of dyadic oppositesex affiliative relationships on a group level by using composite sociality indices (CSI). Further, I will investigate the stability of the strongest affiliative relationships compared to weaker ones across reproductive seasons and across several non-consecutive years. I will also study whether the strength of affiliative relationships has a positive impact on the equitability of directed grooming relationships between individuals by assessing the chance for grooming reciprocation and grooming symmetry on a dyadic level. I will establish the term social bond in Assamese macaque male-female dyads based on the predictions that affiliative relationships are highly differentiated on group level, and the stronger the affiliative relationship the more stable and the more equitable it should be. 
In Chapter 2, I will build on the rationale that Assamese macaques form opposite-sex social bonds. Here, I will investigate in which form females may benefit by forming social bonds with adult males, in terms of reduction of harassment, agonistic support and feeding tolerance. I will assess whether females receive less aggression than expected by chance from a male the stronger the affiliative relationship is. I will test whether the strength of affiliative relationships positively influences male agonistic support towards a particular female, accounting for individual preferences on a dyadic level or individual overall sociality statistically, by using within and between subject designs. I will assess males' feeding tolerance towards females predicted by the strength of affiliative relationships by using cofeeding occurrences of detailed focal tree observations on one study group. Last, I will investigate actual female ingestion rates in the presence of males and test in a within and between subject design the effect of the strength of affiliative relationships on ingestion rates. I will discuss and unite the individual results to establish one main benefit for females that may improve their reproductive fitness.

The study in Chapter $\mathbf{3}$ is building strongly on the general conclusions of the previous chapter that females benefit from forming social bonds with particular males. Based on the rationale that males represent a valuable resource that is in demand (due to quantity and/or quality), females should compete for access to male bonding partners. I will assess in which form females may compete for males and define competitive situations when more than one female shares the male as top partner (based on CSI). I will investigate the influence of female dominance rank on the outcome of a competitive situation. I will assess whether females show enhanced fecal glucocorticoid metabolite levels when being in competitive situations or show reduced levels when being the winner in such a competition. Last, I will explore which males are most likely to be the reason for competition by assessing their current dominance rank in the group and former affiliation with small juveniles, indicating a potentially good father.

Finally, I will summarize and bring together the results of the individual studies in the General Discussion. Here, I will discuss the adaptive significance of opposite-sex affiliative relationships in Assamese macaques and compared to other primates. I will draw a theoretical framework with Assamese macaques as model species, how the human pair-bond may have evolved. Finally, I will draw overall conclusions about my study and suggest future directions. 


\title{
Chapter 1
}

\section{Opposite-sex social bonding in wild Assamese macaques}

\author{
Christine B. Haunhorst ${ }^{a}$, Oliver Schülke ${ }^{a, b} \&$ Julia Ostner ${ }^{a, b}$
}

a Department of Behavioral Ecology, Johann-Friedrich-Blumenbach Institute for Zoology and Anthropology, Georg August University Göttingen, Germany

${ }^{\mathrm{b}}$ Research Group Primate Social Evolution, German Primate Centre, Göttingen 


\begin{abstract}
In large multimale-multifemale primate groups, individual adult males and females may form close social relationships that extend beyond the mating context, a surprising finding for polygynandrous mammals. The patterns of these associations can be relatively stable across time. Here we investigate whether dyadic social relationships between the sexes transcend mere association in wild Assamese macaques and may be characterized as strong, equitable, and stable affiliative relationships or social bonds. We collected $>9,000 \mathrm{hr}$ of focal animal data on adult males and females from two groups of wild Assamese macaques (Macaca assamensis) at Phu Khieo Wildlife Sanctuary, Thailand. Using dyadic composite sociality indices, we found male-female affiliative relationships to be highly differentiated. The stronger the relationships were, the more likely partners were to reciprocate grooming and the more stable were the relationships. In addition, the strongest dyadic relationships remained stable over multiple years as long as both partners remained in the group. These results indicate that in a polygynous species particular males and females form strong, equitable, and enduring affiliative relationships qualitatively similar to the same-sex bonds described for female baboons and male chimpanzees.
\end{abstract}

Keywords: Male-female relationships, social bonds, long-term stability, Assamese macaques 


\section{INTRODUCTION}

The patterns, costs, and benefits of affiliative social relationships are central to understanding fitness and adaptive behavior in socially living mammals (Silk et al., 2003, 2009; Cameron et al., 2009). In primates these relationships can provide benefits for individuals on very different levels, ranging from short term stress reduction to increased longevity (Smuts and Watanabe, 1990; Silk, 1994; Palombit, 2000; Silk et al., 2004; Engh et al., 2006; Ostner et al., 2008; Schülke et al., 2010; Archie et al., 2014; Young et al., 2014a). Primates form affiliative relationships with both same-sex and opposite-sex partners, and may establish and maintain these relationships with the same underlying neuroendocrinological mechanism, the oxytocinergic system (e.g.: Ross and Young 2009; Insel 2010; Crockford et al. 2013). Much of what we know about characteristics and associated benefits of affiliative relationships in primates comes from work on same-sex social relationships in the philopatric sex where coresidency in the same social group is stable (e.g.: Silk et al., 2006a, 2006b; Mitani, 2009). Here, we extend this perspective by asking whether opposite-sex relationships can be characterized as social bonds, i.e. whether relationship stability (the maintenance over time of a partner in the top relationship ranks) and relationship equitability (how balanced the exchange of goods and services is between partners) increase with relationship strength (how often and for how long partners affiliate).

Opposite-sex relationships outside of the context of mating have been extensively studied in several primate taxa (lemurs: Gould 1996; Pereira and McGlynn 1997; Ostner and Kappeler 1999; baboons: Seyfarth 1978; Smuts 1985; Palombit et al. 1997; Lemasson et al. 2008; Nguyen et al. 2009; macaques: Takahata 1982; Chapais 1983a; Chapais 1983b; Hill 1990; Manson 1994; Majolo et al. 2010; Massen and Sterck 2013; Ostner et al. 2013; capuchins: O'Brien 1991; Rose 1994; Perry 1997). In most baboons, close social relationships between a male and a female outside the mating context are limited to the presence of a dependent offspring (Palombit et al., 1997; Nguyen et al., 2009). Olive baboons (Papio anubis) present an exception within the baboons by forming stable opposite-sex relationships that last for longer than one female reproductive cycle (Smuts, 1985). Recently, studies on chimpanzees (Pan troglodytes), rhesus (Macaca mulatta) and Assamese macaques (Macaca assamensis) have shown that opposite-sex relationships may be stable over time (Langergraber et al., 2013; Massen and Sterck, 2013; Ostner et al., 2013).

Close association with a member of the opposite sex may confer fitness benefits (Massen et al., 2010; Archie et al., 2014). Benefits include decreased risk of infanticide or harassment from conspecifics (Takahata, 1982; Palombit et al., 1997; Perry, 1997; Moscovice et al., 2009; Nguyen et al., 2009; Kulik et al., 2011), increased access to food resources (Hill, 
1990), increased mating or paternity success (Smuts, 1985; Ostner et al., 2013), or paternal care provided to the female's offspring (Smuts, 1985; Manson, 1994; Huchard et al., 2010).

The characterization of opposite-sex relationships in some studies is based on spatial association data only (Takahata, 1982; Hill, 1990; Langergraber et al., 2013; Ostner et al., 2013), which lack information on the directionality and quality of direct affiliative interactions necessary to characterize social relationships (Machanda et al., 2013). Studies on female chimpanzees, for example, produced mixed evidence for the relationship between spatial association and affiliative relationships (Gilby and Wrangham, 2008; Lehmann and Boesch, 2009; Foerster et al., 2015), suggesting that spatial association does not always predict a dyad's tendency to affiliate. The pattern of affiliative relationships may influence costs and benefits in both sexes (Chapais, 1983a), making it necessary to use a combination of spatial and affiliative behavior to characterize social relationships (Massen et al., 2010). In same-sex relationships, increasing strength of an affiliative relationship is often associated with increasing stability and equitability in the exchange of services (Silk et al., 2006a, 2006b; Frank and Silk, 2009; Mitani, 2009; Young et al., 2014b; Kalbitz et al., 2016). Relationship strength may lead in turn to increased coalition formation, resulting in acquisition or maintenance of higher dominance ranks within groups (Schülke et al., 2010). Strength, equitability and stability are characteristics that social bonds in animals share with human friendships (Silk, 2002; Massen et al., 2010; Ostner and Schülke, 2014). Without making assumptions about homology or analogy, we assessed whether we could find the same characteristics defining human friendships in opposite-sex affiliative relationships in a non-human primate.

We present data on opposite-sex affiliative relationships in wild Assamese macaques (Macaca assamensis) where group-wide and individual female-male spatial association patterns were stable over several years (Ostner et al., 2013). We used a dyadic composite sociality index to measure the strength of affiliative relationships and assess the differentiation in relationship strength across all possible dyads (Silk et al., 2006a, 2006b, 2010a). The use of the dyadic composite sociality index allows a qualitative comparison to studies on same-sex relationships (Silk et al., 2006a, 2006b; Schülke et al., 2010; Kalbitz et al., 2016). Based on the hypothesis that male-female affiliative relationships represent strong social bonds (Ostner and Schülke, 2014), we derived the following predictions. First, we predicted that stronger affiliative relationships would be more stable than weaker relationships and tested this idea by comparing the within-year seasonal stability of a female's top three male relationships ordered by relationship strength to the stability of her lesser relationships ranking $4^{\text {th }}$ to $6^{\text {th }}$ by strength. Furthermore, we checked whether females maintained their top relationships among three non-consecutive years, separated by two years each, accounting for demographic events that might cause instability. Following Mitani (2009), Silk et al. (2010), and Ostner \& Schülke (2014), we further predicted that the equitability of relationships should increase with strength. We tested this prediction by assessing whether the probability that grooming is reciprocated and 
the degree of grooming symmetry in an opposite-sex dyad were related to the strength of the dyad's affiliative relationship.

\section{METHODS}

\section{Study Site and Population}

The study population inhabits the Phu Khieo Wildlife Sanctuary (PKWS; $>1,600 \mathrm{~km}^{2}, 16^{\circ} 5^{\prime}$ $35^{\prime} \mathrm{N}, 1^{\circ} 1^{\circ} 20^{\prime}-55^{\prime} \mathrm{E}, 300-1,300$ m altitude) in north-eastern Thailand (Schülke et al., 2011). PKWS is part of an interconnected system of eight protected areas, the $6,500 \mathrm{~km}^{2}$ Western Isaan Forest Complex (Grassman et al., 2005) in Chaiyaphum, and harbors a diverse community of large mammals and predators. Diverse types of forest (Borries et al., 2002) cover the study area.

We collected data on one fully habituated group (AS) of wild Assamese macaques in three annual periods (2007/08, 2010/11 and 2013/14) and on a second fully habituated group (AO) in 2013/14. Both groups included several adult males, several adult females and a large number of immatures (Table 1.1). Each annual study period included the mating season (ms) from October through January and the non-mating season (nms) from February to September (Fürtbauer et al., 2010). We refer to both seasons as reproductive seasons, as they correspond with important reproductive events (conception, birth).

The National Research Council of Thailand (NRCT) and the Department of National Parks, Wildlife and Plant Conservation (DNP) (permit 0004.3/3618, 0002.3/2647, 0002/17, $0002 / 2424$ ) authorized the research at the study site in Thailand. Our research adhered to the American Society of Primatologists (ASP) Principles for the Ethical Treatment of non-human primates.

\section{Behavioral Data Collection}

We followed the study groups on approximately 20 days per month from dawn to dusk. We observed all adult males and females in $30 \mathrm{~min}$ focal animal samples using continuous recording (Altmann, 1974). We distributed focal samples on each individual approximately evenly across 6 two-hour time blocks between 6:00 am and 6:00 pm. Averaged across individuals, we observed each male for $50.1 \pm$ SD 9.8 hours $(N=39)$ in each mating season and for $68.2 \pm$ SD 22.0 hours $(N=51)$ in each non-mating season. We observed females for $31.8 \pm$ SD 6.9 hours each $(N=50)$ in each mating season and for $39.6 \pm$ SD 14.1 hours $(N=70)$ 
in each non-mating season (Table 1.1). During focal samples, we recorded start and stop times for three affiliative behaviors: being in close proximity ( $1.5 \mathrm{~m}$ for at least $10 \mathrm{sec})$, body contact, and grooming.

Table 1.1: Average focal observation time (hrs, mean \pm SD) by reproductive season and sex, and number of focal animals in the study groups at the different study periods*.

\begin{tabular}{|c|c|c|c|c|c|}
\hline \multirow[b]{2}{*}{ Time period } & \multirow[b]{2}{*}{$\begin{array}{l}\text { Reprod. } \\
\text { season }\end{array}$} & \multicolumn{2}{|c|}{$\begin{array}{l}\text { Average obs. time } \\
\text { [hrs] }\end{array}$} & \multicolumn{2}{|c|}{$\begin{array}{c}\text { Number of observed } \\
\text { individuals }\end{array}$} \\
\hline & & के & 우우 & के & 우우 \\
\hline Oct 07-Jan 08 & Mating & $49.6 \pm 3.5$ & $30.3 \pm 3.1$ & AS: 11 & AS:12 \\
\hline Feb 08-Sep 08 & Non-mating & $48.1 \pm 8.8$ & $49.5 \pm 7.4$ & AS: 11 & AS: 12 \\
\hline Feb 10-Sep 10 & Non-mating & $49.4 \pm 21.4$ & $49.7 \pm 5.3$ & AS: 10 & AS: 15 \\
\hline Oct 10-Jan 11 & Mating & $44.1 \pm 6.0$ & $39.0 \pm 4.5$ & AS: 10 & AS: 15 \\
\hline Feb 13-Sep 13 & Non-mating & $81.1 \pm 10.4$ & $46.5 \pm 3.0$ & AS: $7, A O: 10$ & AS: $10, A O: 12$ \\
\hline Oct 13-Jan 14 & Mating & $53.5 \pm 12.6$ & $27.8 \pm 5.9$ & AS: $8, A O: 10$ & AS: $11, A O: 12$ \\
\hline Feb 14-Sep 14 & Non-mating & $82.8 \pm 18.4$ & $19.4 \pm 2.4$ & AS: $7, A O: 6$ & AS:11, AO: 10 \\
\hline
\end{tabular}

${ }^{*}$ Group AS underwent a group split in 2012. Observations thereafter continued on the larger daughter group (ASM), which retains the label AS here for convenience.

\section{Behavioral Data Analysis}

We based our analysis on counts of interactions as well as duration of behavioral states. We defined a grooming interaction as one continuous event as long as there were no interruptions longer than $10 \mathrm{sec}$ or changes in the actor vs. recipient roles. Interactions involving close proximity or body contact began (or ended) whenever one individual started (or ended, for longer than $10 \mathrm{sec}$ ) the behavioral state.

We chose the dyadic composite sociality index (CSI; Silk et al. 2006a) to measure the strength of the affiliative relationships between a male and a female relative to the average male-female relationship in a given group and period. We included the total duration (time spent in behavior, expressed as proportion of observation time) and frequency (count of interactions, relative to observation time) of three behaviors, being in close proximity, body contact and grooming, for each dyad. For constructing the CSI we redefined close proximity and body contact slightly. We subtracted the duration of grooming from the duration of body contact and the duration of body contact from the duration of close proximity, as these behaviors are nested into each other. The modified durations of the behaviors were used to calculate the CSI. To standardize at the level of the social group, we divided each behavior by the average across all adult male-female dyads in the group in the given period. We calculated the index as follows: 


$$
C S I_{x y}=\frac{\sum_{i=1}^{b}\left(\frac{f_{i x y}}{f_{i}}+\frac{d_{i x y}}{d_{i}}\right)}{2 b}
$$

Here $b$ is the number of behaviors that contribute to the index ( 6 in the present study), $f_{i x y}$ is the rate of behavior $i$ for the dyad $x y, f_{i}$ is the mean rate of behavior $i$ across all male-female dyads, $d_{i x y}$ is the total duration of behavior $i$ for the dyad $x y$, and $d_{i}$ is the mean duration of behavior $i$ across all male-female dyads. Depending on the question at hand, we defined a period as either one reproductive season within a year, or a one-year period including both reproductive seasons. The index has a minimum of 0 , a mean of 1 and increases with the strength of the affiliative relationship between a male and a female. We ran row-wise matrix correlations in $\mathrm{R}$ with the function 'cor.test' and method set to 'spearman' (version 3.1.2, $\mathrm{R}$ Core Team 2014) for all combinations of the 6 behavioral measures, and found components of the CSI to be significantly positively correlated [15 correlations per reproductive season and group; all $p<0.05$; mean \pm SD (range) $r_{s}$ for each group-season: $0.75 \pm 0.14$ (0.52-0.98)] .

We tested stability of social relationships over time in two ways: first, across consecutive reproductive seasons and second, across multiple years. To test for stability of relationships across reproductive seasons, we calculated a partner stability index (PSI; Silk et al. 2012) based on the ranking of CSI values from the individual's perspective for each reproductive season separately. We followed Silk et al. (2012) and defined as top partners of a female/male those males/females ranking $1^{\text {st }}, 2^{\text {nd }}$ and $3^{\text {rd }}$ by CSI value and as lesser partners the males/females ranking $4^{\text {th }}-6^{\text {th }}$ by CSI value. All females in all periods had at least six males available as partners. Choosing three individuals per class allowed for minor CSI rank changes within a class that may result from measurement errors in the strength assessment. Additionally, it kept the number of individuals per class constant, and left other lower ranking partners as "degrees of freedom" for changes in partner class membership, i.e. additional males not included in either class (Silk et al., 2012). Our use of classes was an analytical convenience: we do not assume here that macaques categorize relationships into classes of strong and lesser, or top versus other. We calculated the PSI for top or lesser partners separately for consecutive reproductive seasons as follows:

$$
P S I=\frac{N * S-U}{N * S-S}
$$

Here $N$ is the number of reproductive seasons in each year in which partner rank orders were evaluated (always 2 because of the gaps between observation years), $S$ is the number of rank slots being evaluated (always 3 , as we considered 3 top and 3 lesser partners) and $U$ is the observed number of unique partners in both reproductive seasons combined. The index ranges between 0 (none of the partners in a class was the same from one reproductive season to the next) to 1 (all 3 partners were retained).

Second, to test for stability across years, we calculated a dyadic composite sociality index (CSI) over an entire one-year period (2007/08, 2010/11, and 2013/14), which included 
both reproductive seasons combined, thus minimizing effects of reproductive state in these inter-annual comparisons. We calculated the number of top three partners in a given one-year period that remained among the top three in the next period (i.e. 2007/08 to 2010/11, 2010/11 to $2013 / 14)$. As a relationship can last only as long as both partners are co-resident in a group, we determined how many of the top three partners that were not retained had emigrated or died in the time between two periods (based on demographic data available on study group; Ostner \& Schülke, unpubl. data). As different females often had the same males as top three partners, the disappearance of a single male could entail the loss of a top partner for several females, which is why the actual number of emigrations/deaths by individual males was smaller than the number of top three partners that emigrated/died. Our analysis included only females that were adult and alive in at least two consecutive periods among the three periods considered (period 1 to $2: N=10$; period 2 to $3: N=8$ ).

We calculated a grooming equality index (GI; Silk et al. 2006b) to assess the relative equitability in grooming exchanges within a dyad for all dyads that groomed at least twice within one reproductive season.

$$
G I=1-\operatorname{abs}\left(\frac{G_{m}-G_{f}}{G_{m}+G_{f}}\right)
$$

Here $G_{m}$ is the amount of time a male actively groomed a female, $G_{f}$ the amount of time a female actively groomed a male and $a b s$ is the absolute value. The index ranges between 0 (completely one-sided) and 1 (entirely symmetrical) for each male-female dyad.

\section{Statistical Analysis}

To assess the stability of partner choice, we used the Wilcoxon signed rank test to compare the PSI values of top $\left(1^{\text {st }}-3^{\text {rd }}\right)$ and lesser $\left(4^{\text {th }}-6^{\text {th }}\right)$ partners with each other. For the year $2010 / 11$, we chose to exclude one male and one female from the analysis, as CSI-values to the opposite sex equaled zero for all but two possible partners in at least one reproductive season and hence did not allow for a reliable ranking of lesser partners.

We assessed the effect of relationship strength on grooming exchanges using two different statistical models as both the likelihood of reciprocating active grooming and the symmetry in the duration of grooming exchanged might contribute to equitability. With the first model, we tested whether the strength of the affiliative relationship between two individuals predicted the likelihood of grooming reciprocation. Using data collated for each dyad and each year-season combination, we used a binomial model of grooming being reciprocated or not (binary variable $1=y e s, 0=$ no) with the strength of the relationship (CSI; Z-transformed) and reproductive season ( $\mathrm{ms} / \mathrm{nms}$ ) as predictors. As the likelihood of grooming reciprocation probably increases with the number of grooming interactions observed, we set the number of dyadic grooming interactions as an offset factor. We included the group and the identities of the dyad, the male and the female as random factors. We included reproductive season 
(ms/nms) in the model because grooming times differ significantly between reproductive seasons (Fürtbauer et al., 2014). Overall, we had 506 male-female dyads with at least one grooming interaction in at least one reproductive season (years represented 2007/08, 2010/11, and 2013/14). We ran the analyses using the function 'glmer' of the statistics package 'Ime4' (version 3.1.2, R Core Team 2014). We checked several diagnostics of model validity and stability (Cook's Distance, DFBetas, DFFits, leverage and Variance Inflation Factors using the functions 'vif' [R package 'car' (Fox and Weisberg, 2011), 'dffits', 'dfbeta' and 'cooks.distance']. We also examined the distribution of residuals, and residuals plotted against fitted values. None of these measures indicated obvious influential cases or deviations from the assumptions of normality and homogeneity of residuals (Quinn and Keough, 2002; Field, 2005). We evaluated the significance of the full model as compared to a null model (comprising only the random effects) using a likelihood ratio test ( $R$ function anova with argument test set to "Chisq"; Forstmeier and Schielzeth 2011).

Second, we tested the effect of relationship strength on the symmetry of grooming within reciprocating dyads only $(N=279)$. We ran a linear mixed model $(L M M)$ to analyze the combined effect of the strength of the relationships (CSI; Z-transformed) on the symmetry in grooming (grooming index, Gl). We included the number of grooming interactions (\#groom) as a fixed factor to control for the potential effect that two individuals are more likely to balance their exchange if they groom more often. We also included the reproductive season as a fixed factor to control for behavioral changes in mating or non-mating season. We powertransformed CSI and \#groom by 0.3 to achieve an approximately symmetrical distribution and reduce influential cases. We ran the analyses using the function 'Imer' of the statistics package 'Ime4' (version 3.1.2, R Core Team 2014). We again included the group and the identity of the dyad, the male and the female as random factors. We checked model validity and stability as in the former model and none of the measures indicated obvious influential cases, nor obvious deviations from the assumptions of normality and homogeneity of residuals. We could not run a model on all grooming dyads (including those with 1 grooming interaction only), because even after several transformation attempts, we were not able to achieve an approximately normal error distribution, which is a prerequisite to use a model with Gaussian error link function (Williams et al., 2013).

For all statistical models we calculated the 'conditional' $R^{2}$, which measures how well the model fits the data (Cohen, 1992). It represents the variance of the results explained by the model (i.e., fixed and random effects) and is calculated as follows ( $V=$ variance; fe = fixed effects; re = random effects; $\mathrm{mr}=$ model results):

$$
R^{2}=\frac{V f e+V r e}{V f e+V r e+V m r}
$$




\section{RESULTS}

\section{Variation in the strength of relationships}

We computed the dyadic composite sociality index for the whole set of male-female dyads across years, reproductive seasons, and groups $(N=1,258)$. The distribution of the CSI was highly positively skewed, with a median of 0.49 , and only $32 \%$ (403) of dyads above average. The top $10 \%$ of dyads had a sociality index above 2.6 (Figure 1.1), indicating that most malefemale relationships were relatively weak and only a few were strong. Across the complete data set of 90 male and 120 female data points (a data point representing an individual in a given group, year, and reproductive season), we could not identify an above average partner in only 12 cases for males and 12 for females, respectively. The strength of the strongest relationship was 3.55 for females and 3.66 for males and the average difference in CSI value from the top partner to the next was 1.63 for females and 1.46 for males, indicative of a strong relationship differentiation. We found that $42 \%$ of males were not the top partner of any female and $53 \%$ of females were not the top partner of any male. Further, $24 \%$ of males and $30 \%$ of females were the top partner of exactly one individual of the opposite sex. Of all individuals in a group in a given reproductive season, maximally $50 \%$ of individuals had the same individual as top partner, the alpha female of group AO in non-mating season 2014 and the alpha male of group AS in all three reproductive seasons of the years 2013 to 2014. A male and female were mutual top partners in 4 to 6 dyads per reproductive season. Whereas in males the difference in CSI value between top and second ranked female partners was comparable in the mating and non-mating season (average difference in CSI values from top to second ranked partner: ms 1.44; nms 1.48), females formed on average stronger and more differentiated top relationships to males during the non-mating season (average difference in CSI values from top to second ranked partner: ms 1.44; nms 1.77).

\section{Stability}

The stability of relationships from one reproductive season to the next within the same year was significantly higher for females' top three partners than for lesser partners ranking $4^{\text {th }}-6^{\text {th }}$ by CSI (Wilcoxon signed rank test: $V=256, p<0.001)$. In $42 \%$ of possible cases $(N=48)$, the female kept the same male as her strongest partner from one reproductive season into the next and more than two thirds of females (71\%) kept the former strongest partner among the top three partners in the next reproductive season. For males' top three partners, the stability from one reproductive season to the next was also significantly higher than for lesser partners (Wilcoxon signed rank test: $V=137, p<0.05)$. In $19 \%$ of possible cases $(N=37)$, the male kept the same female as his strongest partner and $46 \%$ kept the former strongest partner within the top three partners from one reproductive season to the next. The fact that stability among top 
three partners was systematically higher than among lesser partners indicated that top three partner's stability was not a simple consequence of constraints on partner availability.

Females were also rather consistent in their choice of partners from one annual period to another one two years later (Figure 1.2). In $37 \%$ of possible cases $(N=54)$, females kept their strongest partners from one annual period to the next. Death or emigration of the male partner was responsible for the vast majority (85\%) of terminated strong relationships $(N=34)$. Only $15 \%$ of cases involved animals whose continued joint presence would have allowed persistence of a close relationship that they did not retain.

\section{Equitability}

Overall, the data set included 2,204 grooming interactions across 1,123 male-female dyads. We did not observe grooming in $55 \%$ of 1,123 male-female dyads. Those non-grooming dyads $(N=616)$ had an average CSI of 0.4 , while dyads grooming at least once within one reproductive season $(N=507)$ had an average $C S I$ of 2.0. Grooming distribution was not even between the sexes. Females groomed males on average (across all reproductive seasons, years, and groups) twice as much (mean \pm SD $\mathrm{min} / \mathrm{hrs:} 0.15 \pm 0.17$; $\max : 1.22 \mathrm{~min} / \mathrm{hrs}$ ) as vice versa (mean \pm SD $\mathrm{min} / \mathrm{hrs}: 0.08 \pm 0.13$; $\mathrm{max}: 1.32 \mathrm{~min} / \mathrm{hrs}$ ) if grooming at all $(N=507)$. In $68 \%$ of non-reciprocating dyads ( $N=228$; grooming interaction $\geq 1$ ), females were solely responsible for the grooming with a range of grooming rates from 0.01 to 0.11 interactions per hour of observation (mean $\pm \mathrm{SD}$ : $0.02 \pm 0.01, N=228$ ) and grooming duration from a 0.01 to 48.84 seconds per hour (mean \pm SD $\mathrm{min} / \mathrm{hrs}: 0.11 \pm 0.12, N=228$ ). In the $32 \%$ of non-reciprocating dyads $(N=228)$ where males alone were responsible the number of grooming interactions ranged from 0.01 to 0.06 per hour of observation (mean $\pm S D: 0.02 \pm 0.01, N=228$ ) and duration from 0.01 to 13.88 seconds per hour (mean \pm SD $\mathrm{min} / \mathrm{hrs}$ : $0.02 \pm 0.04, N=228$ ). The grooming equality index varied from 0 to 1 with the majority $(65 \%, N=504)$ of dyads exhibiting an index below 0.5 indicating an unbalanced exchange. Still, $18 \%$ of dyads featured an index above 0.75 , indicating a rather balanced grooming relationship. We also found that CSI values in more asymmetric grooming relations $(\mathrm{Gl}<0.5$, mean $\mathrm{CSI}=1.64, N=369)$ were lower than in symmetric $(\mathrm{Gl}>0.5$, mean $\mathrm{CSI}=2.44, N=128)$ grooming relations.

Overall, females spent more time grooming with other females (mean \pm SD min/hrs: ms $1.96 \pm 1.42$, nms $2.96 \pm 1.50$ ) than with males (mean \pm SD min/hrs: ms $1.45 \pm 1.17$, nms $0.49 \pm 0.46$ ) whereas males spent less time grooming with other males (mean \pm SD min/hrs: ms $0.45 \pm 1.17$ ) than with females.

Our model for the likelihood that grooming was reciprocated at least once within one reproductive season in a male-female dyad explained $67 \%$ of the observed variance $\left(R^{2}=0.67\right)$ and was significantly different from the null model with only random factors (ANOVA: $X^{2}=63.11$, $\mathrm{df}=4, p<0.001)$. The strength of the affiliative relationship (CSI) had a strong positive effect on 
the relative chance of grooming reciprocation $\left(\beta \pm S E\right.$ : $1.25 \pm 0.19, z_{500}=6.57, p<0.001$, $\mathrm{OR}=3.50$, lower, upper $95 \% \mathrm{Cl}: 0.95,1.69)$ and reciprocation was more likely in the non-mating than in the mating season $\left(\beta \pm S E\right.$ : $-0.84 \pm 0.29, z_{500}=-2.87, p<0.005$, OR=0.43, lower, upper 95\% Cl:-1.51, -0.32). The odds of grooming reciprocation were higher the stronger the affiliative relationship was, and independently higher in the mating compared to the non-mating season.

Our model of the symmetry of grooming exchanges with the CSI, reproductive season and grooming frequency as predictors was different from the null model with only random factors (ANOVA: $\left.X^{2}=15.89, \mathrm{df}=3, p=0.001\right)$ and explained $26 \%\left(R^{2}=0.26\right)$ of the variance in the grooming equality index. The strength of affiliative relationships had a very small effect on the

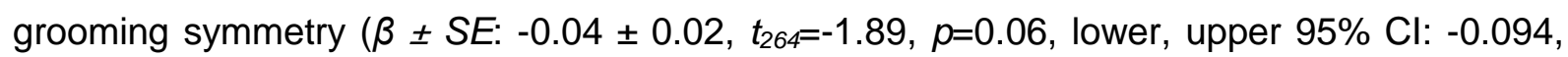
$0.002)$ even after controlling for the effect of grooming frequency on symmetry which was also very weak ( $\beta \pm S E$ : $0.07 \pm 0.02, t_{264}=3.41, p<0.001$, lower, upper $95 \% \mathrm{Cl}: 0.031,0.120$ ).

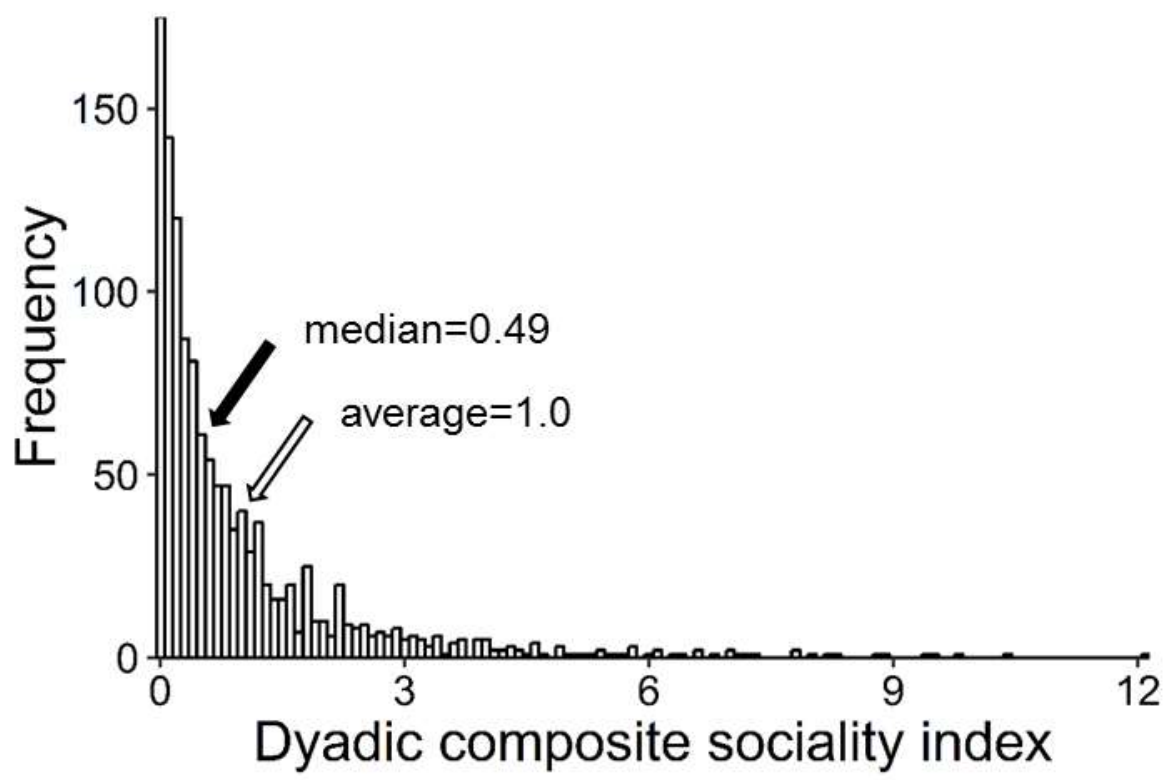

Figure 1.1: Male-female affiliative relationships are highly differentiated as evident from the histogram of the dyadic composite sociality index (CSI) values for all possible male-female dyads $(N=1,258)$. Each unit counts one dyad in each reproductive season, year and group. 


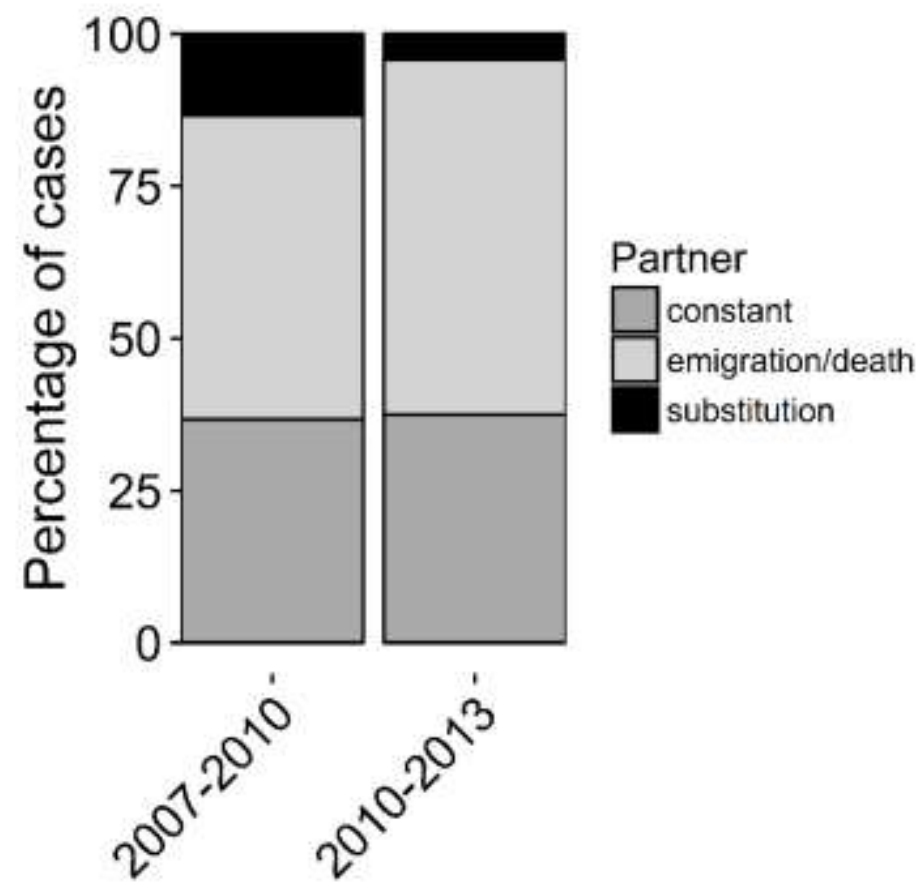

Figure 1.2: Fate of female affiliative relationships with males across multiple years. From one period to the next, the strongest partner was maintained (consistent) or changed either because one partner emigrated or died or despite both partners still residing in the same group (substitution) (2007 to 2010: $N=30 ; 2010$ to 2013: $N=24$ ).

\section{DISCUSSION}

Male and female Assamese macaques in our study population formed affiliative relationships that qualify as strong social bonds by our criteria. A composite measure of relationship strength based on the frequencies and duration of close spatial proximity, body contact and grooming showed pronounced variation among dyads. As predicted, this index was associated positively with the stability of the relationship and with the chance that partners return grooming services. Contrary to our predictions, however, grooming symmetry did not increase with relationship strength.

The distribution of the CSI between male and female Assamese macaques was similar to those of male-male dyads ( $35 \%$ above average; median 0.56; Kalbitz et al. 2016) but more positively skewed than in female-female dyads (44\% above average; median 0.8; Macdonald 2014) of the same population. Cooper and Bernstein (2000) found similar results for another population of Assamese macaques in Assam, India. While females groomed with same-sex 
partners twice as much as males did, grooming in male-female dyads was intermediate (Cooper and Bernstein, 2000). In chimpanzees, where males are the philopatric sex, roles are reversed with males grooming same-sex partners more than females do, whereas grooming between the sexes is intermediate (Machanda et al., 2013). In line with this observation, female-female bonds in chimpanzees are more differentiated than male-male bonds (Langergraber et al., 2009). In female bonded primate groups, female-female affiliative relationships are biased towards close maternal kin, peers, and paternal kin (Cheney and Seyfarth, 1980; Seyfarth, 1980; Silk, 1999; Chapais and Berman, 2004; Silk et al., 2006b; Perry et al., 2008; Schülke et al., 2013; Cords and Nikitopoulos, 2015), with the strength of the relationships declining as a function of relatedness. Because male-female affiliation is usually associated with immediate reproductive benefits (Langergraber et al., 2013; Ostner et al., 2013), positive kin biases in relationship strength are not expected (Pusey, 1987). One may also argue that in contrast to same-sex relationships in the philopatric sex, the number of close or very close partners a female has is not relevant in heterosexual relationships. Females may need only one particular male to reap most benefits from a heterosexual relationship (Fürtbauer et al., 2011a) but the number of partners needed likely depends on the benefits derived. Thus, male-female relationships might be more differentiated than female-female relationships in Assamese macaques and resemble those of the relationships of the dispersing sex.

The stability of relationships is limited partly by the time individuals stay within one social group. If male residency is long, males might spend a significant portion of their adult life in the same group and partners can be chosen on attributes other than just fighting ability/dominance rank (Pradhan and van Schaik, 2008), as seen in Assamese macaques (Ostner et al., 2013). In these monkeys, male tenure is comparatively long, with some adult males remaining in the same social group for several years (Ostner \& Schülke, unpublished data). Additionally, alpha males hold their position for an average of at least 18.3 months (Ostner et al., 2013). Our results indicated that strong relationships last over several years. We also showed that, in most cases, strong opposite-sex relationships ended because of the male's emigration or death. Thus, opposite-sex relationships in this species may not have developed only for the short-term benefits they provide for the partners but for benefits gained over the long run.

Assessing relationship equitability, we found an effect of relationship strength on grooming reciprocation but not on the asymmetry in grooming time among those dyads that showed reciprocal grooming. In female-female relationships, symmetry in grooming exchanges is often viewed as a key aspect of social bonds (Gomes et al., 2009; Schino and Aureli, 2010a). There is an important difference between same- and opposite-sex dyads in terms of asymmetry in partners' resource holding potential. In cercopithecine species, males typically have much higher resource holding potential than females (Dixson et al., 2005), so 
males have the leverage to require more grooming from female partners than vice versa (Barton et al., 1996). In line with this argument, more valuable relationships (based on a 3component analysis) between male and female Japanese macaques (Macaca fuscata) are less symmetric (Majolo et al., 2010) and baboon females are generally responsible for the maintenance of grooming relationships with males (Smuts, 1985; Palombit et al., 1997; Lemasson et al., 2008; Goffe et al., 2016).

One could also argue that grooming equitability is not an ideal measure of how balanced a relationship is because both grooming and being groomed convey benefits (at least for strongly bonded partners). Smuts (1985) called grooming relations in olive baboons more complementary than reciprocal and proposed that females invest more time in grooming males than vice versa to gain benefits from the male's proximity. Both groomer and groomee may experience stress reduction after grooming (measured as self-scratching rates; Aureli and Yates 2010) and the amount of grooming given over intermediate periods may predict fecal glucocorticoid levels whereas grooming received may not (Shutt et al., 2007). It is possible, therefore, that any grooming serves both partners and their bond irrespective of who gives and receives grooming. Alternatively, the failure of closely affiliated partners to exhibit balanced grooming exchanges may result from female competition over access to male partners (Haunhorst et al., n.d.) and females outbidding each other in how much more grooming they give (biological market theory, e.g.: Hemelrijk 1994; Noë and Hammerstein 1995; Barrett et al. 1999).

To date, most studies of heterosexual relationships focused on the immediate benefits males and females may gain from affiliating with the opposite sex, suggesting that both sexes benefit from those relationships in particular ways. As mentioned above several mutually nonexclusive hypotheses may explain the existence of heterosexual relationships, and each is consistent with data from some primate species. The studies investigating these hypotheses have concentrated mostly on the benefits males and females derive from opposite-sex relationships at the time of greatest affiliation (Palombit et al., 1997, 2001; Buchan et al., 2003; Lemasson et al., 2008; Nguyen et al., 2009; Palombit, 2009; Huchard et al., 2010; Moscovice et al., 2010). The collective benefits potentially derived by continuous affiliation over extended periods on the order of reproductive seasons or longer have been little considered. Recent studies of baboons show that social connectedness (Archie et al., 2014) or high quality bonds (Silk et al., 2010b) can enhance the longevity of females, and might even compensate for the fitness losses a female receives from low dominance rank (Silk et al., 2010b). Social connectedness to males can compensate weak integration into the female social network and enhances female longevity in yellow baboons, but the mechanism linking sociality to longevity remains unknown (Archie et al., 2014). To assess costs and benefits of affiliative relationships, one should fully understand the nature of these relationships (Chapais, 1983a). Only if the patterns of social relationships are studied extensively in a diverse set of taxa we will be able 


\section{Male-Female Bonds}

to draw a general conclusion about the importance of sociality. Our study adds to these findings by showing that female same-sex and opposite-sex relationships in wild Assamese macaques share important characteristics, such as differentiation, stability and equitability. 


\section{ACKNOWLEDGEMENTS}

We thank the National Research Council of Thailand (NRCT) and the Department of National Parks, Wildlife and Plant Conservation (DNP) for the permission to conduct this study and for all the support (permit 0004.3/3618,0002.3/2647, 0002/17, 0002/2424). We are grateful to Kanjana Nitaya, Kitti Kreetiyutanont, Mongkul Kumsuk, Jarupol Prabnasuk and Thanee Wongnak (Phu Khieo Wildlife Sanctuary). We thank A. Koenig and C. Borries, who developed the field site at Huai Mai Sot Yai. Special thanks goes to D. Bootros, N. Bualeng, A. Chunchaen, A. Ebenau, I. Fürtbauer, M. Heesen, R. Intalo, S. Jomlotwong, N. Juntuch, J. Kalbitz, M. Karlstetter, T. Kilawit, S. Macdonald, W. Nueorngshiyos, D. Pesek, N. Ponganan, S. Rogahn, P. Saaisawasthikul, K. Srithorn, M. Swagemakers, J. Wanart, B. Whitman, and T. Wisate for their excellent help in the field, and to Andreas Berghänel, Josefine Kalbitz, Marlies Heesen and Sally Macdonald for their support. We are grateful to Marina Cords and two anonymous reviewers for very helpful comments on the manuscript. This research was supported by the Max-Planck Society, and the German Initiative of Excellence through funds to University of Göttingen and the Studienstiftung des Deutschen Volkes to $\mathrm{CBH}$. 


\section{Chapter 2}

\section{Social bonds with males lower the costs of competition for wild female Assamese macaques}

Christine B. Haunhorst $^{a}$, Marlies Heesen ${ }^{a}$, Julia Ostner ${ }^{a, b} \&$ Oliver Schülke ${ }^{a, b}$

a Department of Behavioral Ecology, Johann-Friedrich-Blumenbach Institute for Zoology and Anthropology, Georg August University Göttingen, Germany

${ }^{b}$ Research Group Primate Social Evolution, German Primate Centre, Göttingen 


\begin{abstract}
In polygynandrous mammals, females rarely form affiliative relationships with unrelated males outside of the mating context. The anti-harassment hypothesis provides one explanation for why such relationships have evolved in some gregarious primates: it states that females receive less aggression from group members in the presence of a male partner and gain his support when being harassed by others. This lowers the costs of competition and increases the female's access to resources, as it also reduces interruptions to feeding. Our aim was to test this hypothesis by investigating the benefits that wild female Assamese macaques (Macaca assamensis) in Thailand derive from bonding with particular males. In support of the anti-harassment hypothesis, we found that the strength of affiliative relationships lowered the aggressiveness of a male towards a female, and predicted how much support a female received from a particular male in agonistic interactions with other group members. Additionally, relationship strength predicted the time that females spent co-feeding with a particular male as well as her feeding efficiency in the presence of that male. Hence, female Assamese macaques benefit from forming a social bond to a particular male by reducing the costs of competition.
\end{abstract}

Keywords: agonistic support, competition, feeding tolerance, female benefits, male-female relationships, social bonds 


\section{INTRODUCTION}

The formation of temporarily stable but non-monogamous social bonds between males and females is rare among mammals in general, but relatively common in primates (Chapais, 1983a; Smuts, 1985; Palombit et al., 1997; Lemasson et al., 2008; Nguyen et al., 2009; Goffe et al., 2016; Haunhorst et al., 2016). From the female perspective one explanation for the formation of exclusive social (but not necessarily sexual) bonds to one member of the opposite sex is provided by the anti-harassment hypothesis (Smuts, 1985). This hypothesis states that females receive less harassment in the presence of a male partner and may even receive agonistic support by the male when being harassed by group members (Smuts, 1985; Palombit et al., 1997; Lemasson et al., 2008; Nguyen et al., 2009). Reduced harassment and agonistic support by particular males may also result in lowered costs of competition with other group members.

While close and long-term affiliative same-sex relationships in primates are characterized by an increased degree of equitability in services exchanged (Silk, 2002; Ostner and Schülke, 2014), the exchange of grooming in bonded opposite-sex dyads is never fully balanced as females typically invest more than males (Chapais, 1983b; Smuts, 1985; Ratnayeke, 1994; Palombit et al., 1997; Majolo et al., 2010; Haunhorst et al., 2016). This imbalance in grooming may be compensated by males providing other commodities (Hammerstein and Noë, 2016).

In this study, we tested how close affiliative relationships, here also called opposite-sex social bonds, affected the exchange of services by males towards females. We examined whether a pre-existing relationship provides any positive effects for the female partner in terms of agonistic support and increased access to food resources (Tiddi et al., 2011; Huchard et al., 2013). In the present study, we did not investigate any short-term reciprocation.

Agonistic support is a suitable measure of cooperative behaviour as it involves a clear cost to the donor (time, energy, risk of injury) and a clear benefit for the recipient (Fraser and Bugnyar, 2012), while the costs and benefits of reduced harassment and increased feeding tolerance are more difficult to assess. Yet, all three components are likely to increase lifetime reproductive success in females. Injuries inflicted by any group members might lower a female's physical condition. Nevertheless, males may cause more severe injuries than females in species with pronounced sexual dimorphism in body size and weaponry (Smuts, 1985; Chapais, 1986) where sexual harassment is common (MacCormick et al., 2012). Being the recipient of aggression also increases a female's physiological stress levels which can affect ovarian function and may lead to spontaneous abortion (Wasser and Starling, 1988; Clutton-Brock and Huchard, 2013b; Dettmer et al., 2015), while also having a general negative 


\section{Female Benefits}

effect on female body condition with down-stream consequences for offspring fitness and survival. Male support in agonistic interactions might also benefit a female's dependent offspring, protecting it against potentially infanticidal males (Palombit et al., 1997; Palombit, 1999, 2009).

Feeding competition among females is almost ubiquitous among gregarious primates (Schülke and Ostner, 2012). Female reproductive success is thought to be limited mainly by access to safety and the amount of energy available for reproduction; apart from thermoregulation, gestation and lactation place the highest energy demands on female mammals (Trivers, 1972; van Schaik and van Noordwijk, 1985; Schneider, 2004). Increased food intake can explain enhanced female reproductive success in provisioned groups compared to wild populations of the same species, both in terms of average birth rate and infant mortality (Sugiyama and Ohsawa, 1982; Watanabe et al., 1992). Access to food resources and relaxed feeding environments can therefore have a direct influence on female fitness.

Assamese macaques (Macaca assamensis) are a suitable study system to investigate the benefits that females derive from social bonding with particular males. They live in large multimale-multifemale groups and have a polygynandrous and seasonal mating system. Female philopatry and male migration reduces the likelihood of male relatedness to resident females, which removes the need to consider inclusive fitness benefits in the interpretation of results. Assamese macaques are highly frugivorous and allocate about one third of daily activity time to feeding (Schülke et al., 2011). As both female energy intake and conception rates increase with increasing food abundance it is likely that access to food affects female fitness (Heesen et al., 2013).

We have shown that within Assamese macaque social groups specific adult males and females form strong and enduring affiliative relationships (social bonds) with each other and that these are characterized by frequent and long bouts of grooming and time spent in close proximity (Haunhorst et al., 2016). Both males and females show pronounced variation in the strength of the affiliative relationships they form with members of the opposite sex. The strongest bonds are independent of female reproductive state and may last for several years. Typically, individuals show a strong differentiation of relationship strength between the strongest partner and the others, yet most individuals form strong (above average) relationships to more than one member of the opposite sex. Only half of the males in a group are the top partner of at least one female, and up to five females may share the same top male partner.

Male Assamese macaques benefit from a high degree of spatial association with a female by increased mating success with that female (Ostner et al., 2013). How females benefit from bonding with particular males remains unclear. Nevertheless, a previous study has found that females invest more in the formation or maintenance of opposite-sex bonds than do males 
(Haunhorst et al., 2016). In particular, mutual grooming relations between the sexes are not balanced (Haunhorst et al., 2016), leading to the assumption that males may provide other services in return. We therefore predicted that the stronger the affiliative relationship, 1) the lower the frequency of aggression received by a male and 2) the higher the agonistic support from a male during harassment by other group members. Next, we predicted that with increasing strength of affiliative relationships 3 ) the male's tolerance towards a particular female in the context of feeding increases (i.e. the female can spend more time co-feeding with the male), and 4) that, consequently, a female's food intake rate increases (i.e. a more relaxed feeding environment). As closely bonded partners spend more time in close proximity to each other, we ruled out the possibility that results are driven by proximity alone, by controlling the tests of predictions 2) to 4) for the time that dyad members spent within $5 \mathrm{~m}$ of each other.

\section{METHODS}

\section{Study Site and Population}

We conducted the study at Phu Khieo Wildlife Sanctuary (PKWS; $16^{\circ} 5^{\prime}-35^{\prime} \mathrm{N}, 1^{\circ} 1^{\circ} 20^{\prime}-55^{\prime} \mathrm{E}$, $300-1,300 \mathrm{~m}$ ) in north-eastern Thailand (Schülke et al., 2011). The study area is covered by hill evergreen forest, dry evergreen forest, dry dipterocarp, and bamboo stands (Borries et al., 2002). PKWS is part of an interconnected system of eight protected areas, the $6500 \mathrm{~km}^{2}$ Western Isaan Forest Complex (Grassman et al., 2005) in Chaiyaphum, and harbors a diverse community of large mammals and predators.

We collected data on one fully habituated group AS of wild Assamese macaques in 2007/08, 2010/11 and 2013/14, and on a second fully habituated group AO in 2013/14. Each study period captured one year, including the mating season (ms) from October through January and the non-mating season (nms) from February to September (Fürtbauer et al., 2010). At any time, both groups included several adult males, several adult females and a large number of immatures (see Table 2.1 for details of subjects).

\section{Behavioral Data Collection}

We followed the study groups on approximately 20 days each month from dawn to dusk. We observed all adult males and females in $30 \mathrm{~min}$ focal animal samples using continuous and instantaneous recording (Altmann, 1974). In the continuous protocol we recorded all social 
interactions, including grooming, body contact, approaches into and departures from a distance of $1.5 \mathrm{~m}$ to other individuals and vice versa (details in Haunhorst et al., 2016), and agonistic interactions with details on direction and duration of the behaviour. We defined agonistic interactions as interactions between two or more individuals when at least one of the individuals displayed directed aggressive behaviour (lunge, chase, slap, push \& pull, bite, ground slap) towards another. We also recorded all individuals within $5 \mathrm{~m}$ of the focal animal instantaneously every 10 min during the focal sample.

Table 2.1: Average focal animal observation times (mean \pm standard deviation [SD], hrs), and number of observed adult individuals per year and sex*.

\begin{tabular}{|l|l|l|l|l|}
\hline & \multicolumn{2}{|l|}{ Number of individuals } & \multicolumn{2}{l|}{ Average obs. time [hrs] } \\
\hline Time period & Male & Female & Male & Female \\
\hline Oct 2007 - Sep 2008 & AS:11 & AS: 12 & $97.7 \pm 8.5$ & $79.9 \pm 7.9$ \\
\hline Feb 2010 - Jan 2011 & AS: 10 & & $93.9 \pm 20.3$ AS: 15 & $88.7 \pm 8.5$ \\
\hline Feb 2013 - Jan 2014 & AS: 6, AO: 10 & AS: 10, AO: 12 & $123.3 \pm 35.6$ & $74.6 \pm 4.4$ \\
\hline
\end{tabular}

* Study group AS underwent a group split in 2012. The larger subgroup ASM has been observed here and for convenience is labelled AS throughout. The table does not represent the demographic situation fully, as we did not include males and females that were not continuously observed over the whole period. For this study, we considered only individuals that were resident in the group and available for focal observation for the whole year of the respective study period.

\section{Focal tree sampling}

We collected focal tree data in two of the three study periods on group AS (January-September 2008 and July 2010-April 2011), using a modified version of the "focal tree method" (Vogel and Janson, 2007). The advantage of this method is that it allows for the observation of all individuals feeding in a food patch simultaneously, combining information on patch characteristics, identity of co-feeders, food-related agonism and individual feeding behavior. We defined a food patch as an individual tree, large shrub or liana with a separate crown or as individuals of the same species with overlapping crowns (Leighton and Leighton, 1982; Strier, 1989; Phillips, 1995; Sterck et al., 1997). A focal tree observation, conducted by two observers, started as soon as we observed a monkey entering a patch to feed and ended when the last individual left the patch and the group had moved on.

During period 1 (2008), we recorded the number and identity of all monkeys in the patch at 5-min intervals. During period $2(2010 / 11)$, we recorded the exact time to the minute and identity of each monkey entering and leaving the food patch instead, and from this, we calculated the number and identity of all monkeys in the patch at 5-min intervals. Additionally, we conducted 1 -min focal samples of as many adult females as possible, recording the number of food items ingested per minute (for more details see Heesen et al. 2014). 


\section{Behavioral Data Analysis}

Composite sociality index (CSI)

We chose the dyadic composite sociality index (CSI; Silk et al. 2006a) as a measure of the strength of the affiliative relationship between a male and a female relative to the group's average across all male-female relationships for a given group and period (i.e. one year). We included the total duration (time spent in respective behavior) and frequency (count of interactions) of three behaviors, being in close proximity $(<1.5 \mathrm{~m})$, body contact and grooming, for each dyad. We assigned equal weights to each component but subtracted nested behaviors from each other (i.e. time in close proximity without time in body contact and time in body contact without time grooming). All elements of the CSI were significantly positively correlated (Haunhorst et al., 2016). We calculated all terms per hour of dyadic observation to control for observational errors and divided each dyadic component by the group's average to ensure comparability between observation periods (for details see Haunhorst et al. 2016). We tested the variability of each included factor to rule out the possibility of one of the factors biasing the index and found that in each category the maximum value was about ten times as high as the average (mean \pm SD: $9.67 \pm 1.97$ ), without much variation between the different factors from which the index was calculated.

\section{Dominance hierarchy}

To compute a dominance hierarchy, we used all decided dyadic agonistic interactions where clear submissive signs (make room, silent bared teeth, unprovoked give ground) were observed from both continuous focal protocols and ad libitum data. We calculated a separate hierarchy for both males and females respectively based on the adjusted normalized David's Score (Schmid and De Vries, 2013) for each one-year period of data collection (Table 2.2).

Table 2.2: Details on characteristics of dominance hierarchies: number of dyads (dyad), number of decided dyadic conflicts (confl), directional consistency index (DCI) and \% unknown relationships (unk. rel) for both groups and all years for males and females separately.

\begin{tabular}{|c|c|c|c|c|c|c|c|c|}
\hline \multirow{2}{*}{$\begin{array}{l}\text { Group } \\
\& \\
\text { Period }\end{array}$} & \multicolumn{4}{|c|}{ Males } & \multicolumn{4}{|c|}{ Females } \\
\hline & $\begin{array}{l}\text { No. of } \\
\text { dyads }\end{array}$ & $\begin{array}{l}\text { No. of } \\
\text { confl }\end{array}$ & $\begin{array}{l}\text { \% unk. } \\
\text { rel }\end{array}$ & $\mathrm{DCl}$ & $\begin{array}{l}\text { No. of } \\
\text { dyads }\end{array}$ & $\begin{array}{l}\text { No. of } \\
\text { confl }\end{array}$ & $\begin{array}{l}\text { \% unk. } \\
\text { rel }\end{array}$ & $\mathrm{DCl}$ \\
\hline $\begin{array}{l}\text { AS } \\
2007 / 08\end{array}$ & 66 & 361 & 12.1 & 0.93 & 66 & 249 & 10.6 & 0.96 \\
\hline $\begin{array}{l}\text { AS } \\
2010 / 11\end{array}$ & 36 & 240 & 13.9 & 0.94 & 105 & 393 & 12.4 & 0.96 \\
\hline $\begin{array}{l}\text { AS } \\
2013 / 14\end{array}$ & 21 & 165 & 0 & 0.95 & 55 & 398 & 9.1 & 1 \\
\hline $\begin{array}{l}\text { AO } \\
2013 / 14\end{array}$ & 55 & 292 & 10.9 & 0.99 & 78 & 570 & 10.3 & 0.98 \\
\hline
\end{tabular}




\section{Aggressiveness}

We calculated the aggressiveness within a relationship by computing an index that reflected how much aggression a female received relative to an expected value. First, we calculated the frequency of aggression received by a female from all males per hour of observation. To derive an expected value we allocated bouts of aggression against a female according to the time a male spent within $5 \mathrm{~m}$ of the female relative to the time other males spent within $5 \mathrm{~m}$ of the same female. Finally, we calculated the deviation of the observed frequency of aggression received from a particular male to the expected frequency by subtracting the latter from the former. Positive values therefore indicate that a female received more aggression from a particular male than expected. It should be noted that no female was ever seen to direct aggression towards a male.

\section{Coalitionary aid}

To assess how likely a male was to support a female during agonistic interactions, we considered all continuous behavioral data from both males and females as well as data taken ad libitum in the field. Overall, we found $57 \%$ of all reported cases of support in the continuous behavioral data. We considered only aggressive interferences in on-going dyadic conflicts among adults where the male directed aggression against one opponent in favor of a female $(N=216)$.

\section{Food acquisition}

We counted each individual only once per focal tree observation, even when the individual was feeding for several 5-min scans. We defined co-feeding as a male and a female feeding in the same food patch at the same time. We also counted each dyad only once per focal tree observation, regardless of the actual time spent feeding together. We calculated a standardized term for the frequency of co-feeding by a dyad to correct for observation effort, as follows:

$$
\text { corrected co }- \text { feeding }=\frac{f m \text { cofeed }}{(f \text { feed }+m \text { feed })-f m \text { cofeed }}
$$

Here, fm cofeed is the number of observations dyad fm is feeding together, $f$ feed is the number of observations individual $f$ was feeding and $m$ feed is the number of observations individual $\mathrm{m}$ was feeding.

We recorded female ingestion rates by counting the number of bites taken from a large item, the number of complete items (e.g. leaves, flowers, or fruit) or the number of handfuls of very small items (e.g. bunches of leaves) ingested during 1 min of continuous feeding. Because of differences in bite size, food handling time and food distribution within patches, ingestion rates can vary greatly across food species and items. We therefore standardized female ingestion rates for each food species and item to enable comparisons across food species, as 
follows: we calculated the mean female ingestion rate for each food species and item (across the group) and divided the focal female's observed ingestion rate by the calculated average. In this way, we could assess the influence of individual co-feeders on a female's ingestion rate.

Table 2.2: Details on characteristics of dominance hierarchies: number of dyads (dyad), number of decided dyadic conflicts (confl), directional consistency index (DCI) and unknown relationships (unk. rel) for both groups and all years for males and females separately.

\begin{tabular}{|l|c|c|c|c|c|c|c|c|}
\hline $\begin{array}{l}\text { Group } \\
\begin{array}{l}\boldsymbol{\&} \\
\text { Period }\end{array}\end{array}$ & \multicolumn{4}{|c|}{ Males } & \multicolumn{4}{c|}{ Females } \\
\cline { 2 - 9 } & dyad & confl & unk.rel & DCl & dyad & confl & unk.rel & DCI \\
\hline $\begin{array}{l}\text { AS } \\
\text { AS }\end{array}$ & 66 & 361 & 12.1 & 0.93 & 66 & 249 & 10.6 & 0.96 \\
\hline $\begin{array}{l}\text { AS } \\
\mathbf{2 0 1 0 / 1 1}\end{array}$ & 36 & 240 & 13.9 & 0.94 & 105 & 393 & 12.4 & 0.96 \\
\hline $\begin{array}{l}\text { AS } \\
\mathbf{2 0 1 3 / 1 4}\end{array}$ & 21 & 165 & 0 & 0.95 & 55 & 398 & 9.1 & 1 \\
\hline $\begin{array}{l}\text { AO } \\
\mathbf{2 0 1 3 / 1 4}\end{array}$ & 55 & 292 & 10.9 & 0.99 & 78 & 570 & 10.3 & 0.98 \\
\hline
\end{tabular}

\section{Statistical Analysis}

\section{General procedure}

We conducted statistical analysis using R 3.1.2 (version 0.98.1087; R Core Team 2014). We ran Linear Mixed Models (LMM) with Gaussian error link function and Generalized Linear Mixed Models (GLMM) with Poisson error link function using the package 'Ime4' (Bates et al., 2014). Prior to analysis, we transformed some covariates (CSI, PROX) with the power of 0.3 (log-transformation not being possible, as minimum of both factors could be zero) to reduce influential cases by achieving symmetrical distributions of the predictors. We standardized all covariates by the variance-stabilizing z-transformation to achieve comparable estimates (standardized betas). We applied several diagnostics of model validity and stability [Variance Inflation Factors of reduced models without random factors (function 'vif' of the R package car; Fox and Weisberg 2011); visual inspection of distribution of residuals and residuals plotted against fitted values (Quinn and Keough, 2002); overdispersion and model stability, using functions provided by Roger Mundry (Leipzig)] and found no obvious influential cases, nor obvious deviations from the assumptions of normality and homogeneity of residuals (Quinn and Keough, 2002; Field, 2005). To test the significance of the predictors as a whole we compared the fit of the full model with that of the null model comprising the intercept and all random factors only by using a likelihood ratio test ( $R$ function anova with argument set to "Chisq"; (Dobson, 2002; Forstmeier and Schielzeth, 2011). We found all presented full models to be significantly different from the respective null models (Table 2.4). In all following models 
male and female ID (crossed factors), as well as group and period ID (nested factors) were included as random factors.

We used 'within-subject' and 'between-subject' designs to account for, respectively, the individual preferences and overall sociality of an individual. While the CSI is calculated for all dyads in a group, each individual can differ in the resulting average strength of its relationships with others as well as the range of CSI values it exhibits between its weakest and strongest relationships. To account for both an individual's overall sociality as well as its individual preferences in terms of the skew in the strength of its individual relationships to others, we did not alter the index itself but rather the form in which it appeared for each individual in the statistical models. We first calculated each individual's mean CSI across all her relationships with a member of the opposite sex as a measure of its 'overall sociality'. Next, we calculated the deviation of the CSI values of each individual's relationships with each opposite-sex individual from its mean CSI to calculate 'individual preferences' on a dyadic level. This approach allowed us to assess overall sociality and individual preferences of each individual in the group, rather than group wide patterns.

For all statistical models we calculated the 'marginal' $R^{2}$ using the package MuMln with the argument set to 'r.squaredGLMM' (Bartoń, 2013).

\section{Model 1: Aggressiveness}

To assess whether the aggressiveness within a relationship was related to the strength of the relationship we used a LMM with aggressiveness as response and CSI as the predictor (Table 2.3).

\section{Model 2: Aid}

To model the effect of the CSI on the probability that a female gains support from a particular male we ran a GLMM. The response in this model was the count of supportive coalitions by a male towards a female within each one-year period (Table 2.3). We predicted that for each individual female, the coalitionary support received from a particular male should be predicted by the female's 'individual preferences', and that her 'overall sociality' should predict the overall amount of aid she received from all males. We used both 'individual preference' and 'overall sociality' of females as predictors in the model. We included male dominance rank as a fixed factor, as male rank is expected to predict his ability to support females against others, particularly other males. We included the time that members of each dyad spent within $5 \mathrm{~m}$ (PROX; which is not included in the CSI) as a fixed factor to control for an opportunity effect and to exclude the possibility that the mere presence of a male in the female's proximity would be enough for her to receive his support. We found no obvious violations of overdispersion (overdispersion parameter $=0.84, X^{2} 447=375.56, p=0.994$ ). 
Table 2.3: Summary of all presented models with response, predictors and fixed control factors with comparison of full vs. null models. All models included male and female ID, as well as group and year as random factors. We compared models by using ANOVA.

\begin{tabular}{|l|l|l|l|l|l|l|}
\hline Model name & Response & Predictors & $\begin{array}{l}\text { Control } \\
\text { factors }\end{array}$ & $\boldsymbol{X}^{\mathbf{2}}$ & df & $\boldsymbol{P}$ \\
\hline $\begin{array}{l}\text { Aggressivenes } \\
\text { s }\end{array}$ & Aggressiveness & CSI & 41.8 & 1 & $<0.001$ \\
\hline Aid & $\begin{array}{l}\text { No. of agonistic } \\
\text { support }\end{array}$ & $\begin{array}{l}\text { Female } \\
\text { 'individual } \\
\text { preference' } \\
\text { and 'overall } \\
\text { sociality' }\end{array}$ & $\begin{array}{l}\text { Time spent } \\
\text { in 5m, male } \\
\text { dominance } \\
\text { rank }\end{array}$ & 711. & 4 & $<0.001$ \\
\hline Co-feeding & $\begin{array}{l}\text { Corrected co- } \\
\text { feeding }\end{array}$ & $\begin{array}{l}\text { Male } \\
\text { 'individual } \\
\text { preference' } \\
\text { and 'overall } \\
\text { sociality' }\end{array}$ & $\begin{array}{l}\text { Time spent } \\
\text { in 5m }\end{array}$ & 39.8 & 3 & $<0.001$ \\
\hline Ingestion rate & $\begin{array}{l}\text { Average } \\
\text { standardized } \\
\text { ingestion rate }\end{array}$ & $\begin{array}{l}\text { Female } \\
\text { 'individual } \\
\text { preference' } \\
\text { and 'overall } \\
\text { sociality' }\end{array}$ & $\begin{array}{l}\text { Female } \\
\text { dominance } \\
\text { rank, co- } \\
\text { feeding } \\
\text { group size }\end{array}$ & 15.0 & 4 & $<0.005$ \\
\hline
\end{tabular}

Model 3: Co-feeding

To model the effect of relationship strength on the food acquisition of females we chose two approaches. In our first model, we predicted that the strength of the social relationship between females and males would increase the female's ability to feed in the male's presence - by increasing the male's tolerance (Table 2.3). For this reason, we used 'individual preference' and 'overall sociality' of males here. We built a LMM, using the corrected co-feeding as response, and 'individual preference' as well as 'overall sociality' of males as predictors. We controlled for the time the members of each dyad spent within $5 \mathrm{~m}$ to ensure that co-feeding events were not merely due to opportunity.

Model 4: Ingestion rate

As a second approach to assessing the consequences of co-feeding, we assessed how much food a female ingested in the presence of different males and if this quantity (measured by ingestion rates) increased with the strength of the female's relationship to the male which she was co-feeding with (Table 2.3). We constructed an LMM and used standardized ingestion rates as the response, and 'individual preference' as well as 'overall sociality' of females as predictors. We included the female's dominance rank and the size of the feeding group as fixed factors into the model. We did so because female dominance rank might have an independent effect on her feeding behavior, as low-ranking females face a higher degree of feeding competition than high-ranking females, and feeding group size might influence vigilance and thus feeding efficiency. 


\section{RESULTS}

\section{Aggressiveness}

We tested the aggressiveness within affiliative relationships in 462 male-female dyads. We observed $0.03 \pm 0.02$ SD agonistic interactions per hour of observation in each dyad. In 36 dyads, we observed no agonistic interactions.

The aggressiveness model showed that the stronger the affiliative relationship between a female and a male (measured as CSI) the smaller the amount of aggression observed $(\beta \pm \mathrm{SE}$ : $-0.32 \pm 0.04, t=-9.11, p<0.001)$ compared to the amount expected based on the time each dyad spent in a $5 \mathrm{~m}$ distance (Figure 2.1). The only predictor of the model explained $11 \%$ of the variance in the relationship's aggressiveness $\left(m R^{2}=0.11\right)$.

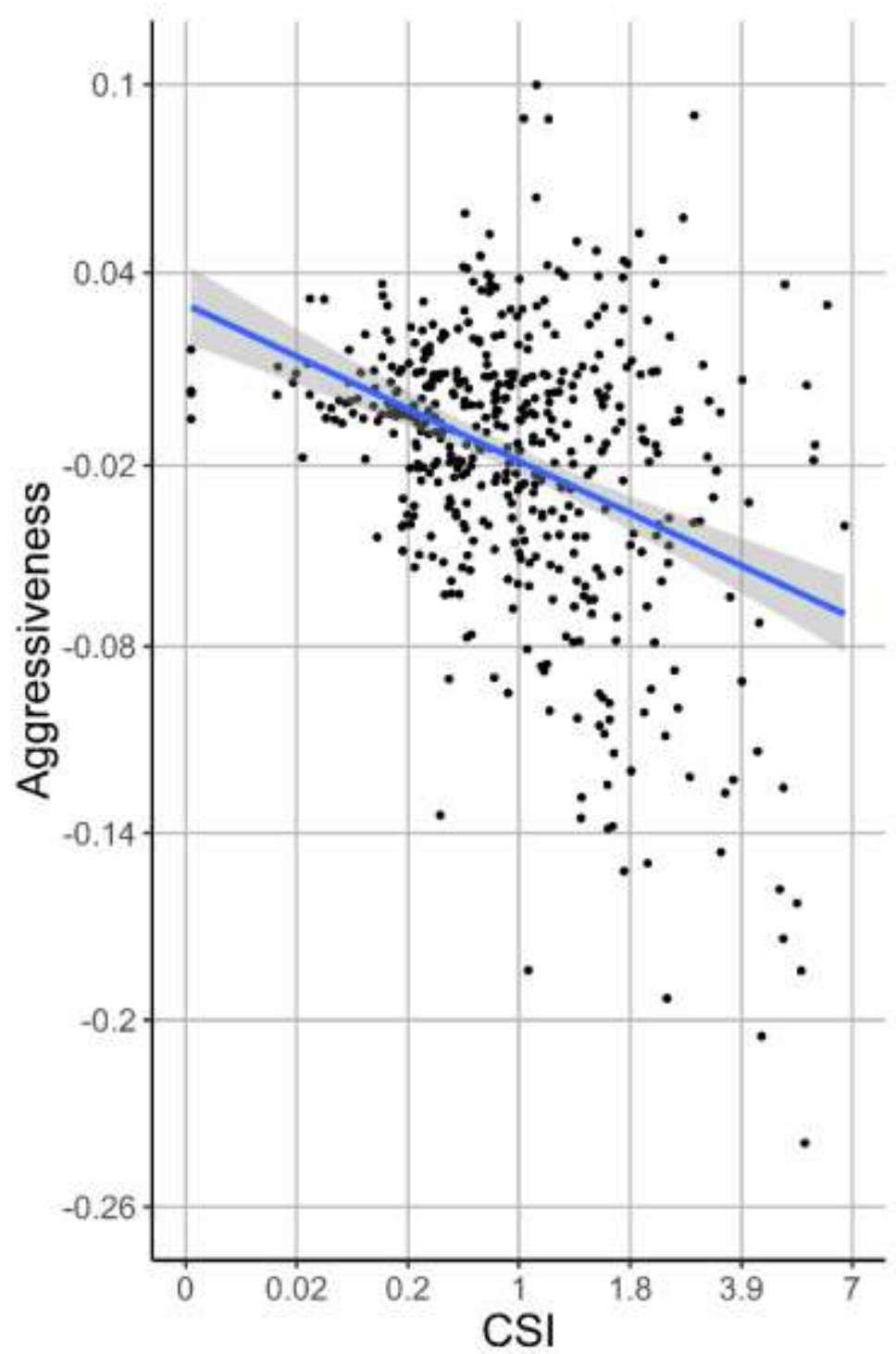

Figure 2.1: The effect of the strength of a dyadic affiliative relationship (CSI) on the aggressiveness within a relationship. The blue line depicts the model and the shaded area indicates bootstrapped $95 \%$ confidence intervals (R package 'ggplot2'; Wickham \& Chang, 2016). Both axes were back transformed to the original values, with the CSI on a logarithmic scale. 
Coalitionary support by males

Over all dyads across all periods and groups $(N=452)$ we found 216 cases of male support during a conflict. Males supported females against other males in 174 cases. In only 42 cases did males support one of the antagonists in an on-going conflict between females. The supported female won in all but one of the 216 episodes.

Our model showed that the opportunity to support a female was a significant predictor of the number of male interventions in a female's conflicts; the time a female spent within $5 \mathrm{~m}$ of a particular male was positively related to the amount of support the female received from him ( $\beta \pm S E: 0.18 \pm 0.05, z=3.53, p<0.001)$. With opportunity accounted for, the model indicated that the strength of a social relationship was positively associated with the probability that a male would support a female. Female 'individual preference' was a highly significant correlate of coalitionary support ( $\beta \pm S E$ : $0.42 \pm 0.11, z=3.84, p<0.001$; Figure 2.2), while 'overall sociality' did not increase the amount of support a female received $(\beta \pm S E: 0.30 \pm 0.23, z=1.32$, $p=0.187)$. A male's dominance rank predicted the likelihood of his support for females $(\beta \pm S E$ : $0.59 \pm 0.14, z=4.15, p<0.001)$. This is particularly important, as most male-female coalitions were directed against other adult males, the clear majority (98\%) directed against lower ranking males and the top tree dominant males provided $75 \%$ of the support (Figure 2.3). Male support of females was equally divided between mothers and non-mothers (mother: $N=116$, nonmother: $N=100)$. Overall, the fixed effects of the model explained $37 \%$ of the variance in coalitionary support $\left(m R^{2}=0.37\right)$. 

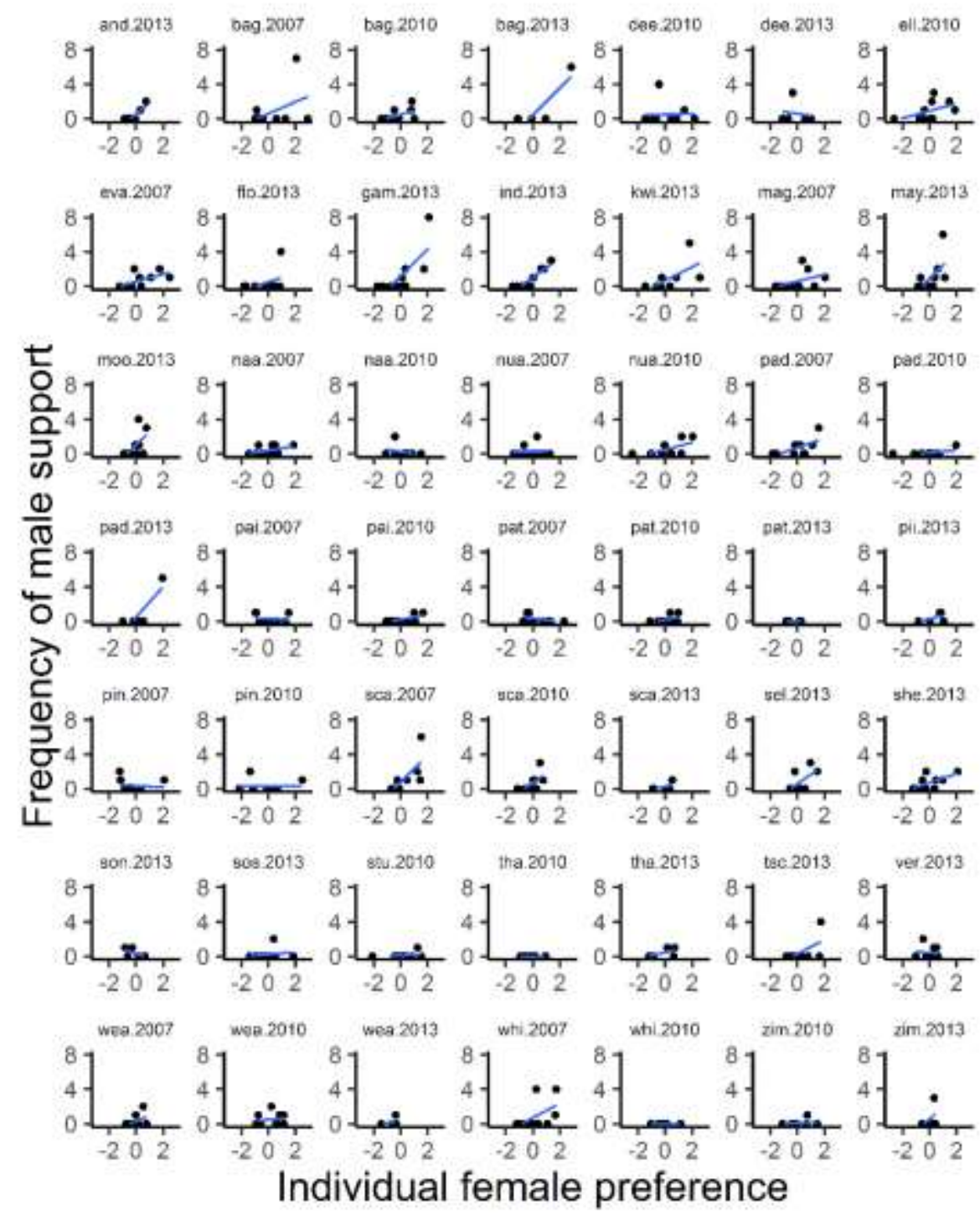

Figure 2.2: Coalitionary support received by females from different males is predicted by the strength of the partners' social bond (CSI). The x-axis shows the transformed 'individual preference' of each female and the $y$-axis the actual number of supports received by a male. We ordered females first alphabetically and then chronologically. The blue lines indicate the linear regression predicted by the model for each individual. Above each graph the female ID and observation period is given. 


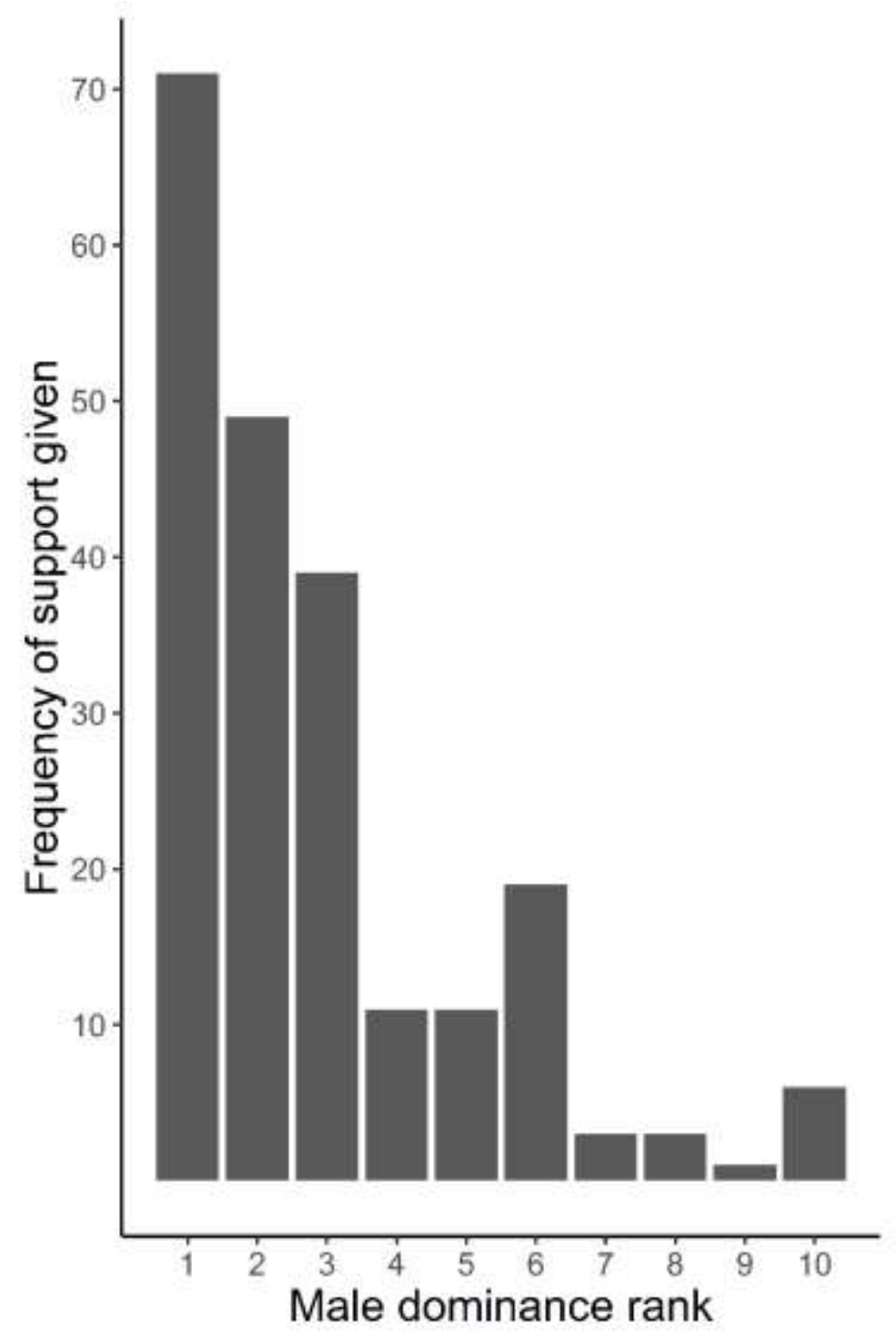

Figure 2.3: Distribution of agonistic male support across males of different dominance ranks.

\section{Food acquisition}

We analyzed 422 focal tree observations in this model (2008: $N=255 ; 2010 / 11: N=167$ ), assessing 252 different male-female dyads. Each male appeared in $46.4 \pm 32.9$ SD, and each female in $51.2 \pm 26.4$ SD focal tree observations.

The fixed effects in the model explained $37 \%$ of the variance in the observed time a dyad spent co-feeding $\left(m R^{2}=0.37\right)$. The higher the 'overall sociality' of a male, the more likely he was observed co-feeding with females ( $\beta \pm \mathrm{SE}$ : $0.11 \pm 0.02, \mathrm{t}=4.85, p<0.001)$. Females were more likely to be observed co-feeding with a male the stronger their affiliative relationship was ( $\beta \pm \mathrm{SE}: 0.02 \pm 0.00, \mathrm{t}=4.49, p<0.001$ ), reflecting increased male tolerance towards them 
(Figure 2.4). These effects are independent of pure spatial effects measured as the time dyad members were within $5 \mathrm{~m}$.

The fixed effects in the model describing the effect of relationship strength on female feeding efficiency explained only $8 \%$ of the variance of our results $\left(m R^{2}=0.08\right)$. Neither feeding group size $(\beta \pm S E:-0.01 \pm 0.03, t=-0.18, p=0.880)$, nor female dominance rank ( $\beta \pm S E: 0.18 \pm$ $0.11, \mathrm{t}=1.54, p=0.136$ ), had an effect on a female's food ingestion rates. Both female 'individual preference' ( $\beta \pm \mathrm{SE}$ : $0.14 \pm 0.06, \mathrm{t}=2.16, \beta=0.038$ ) and 'overall sociality' ( $\beta \pm \mathrm{SE}$ : $0.48 \pm 0.19$, $\mathrm{t}=2.49, p=0.014)$ were positively associated with the number of food items or bites recorded from a female in the presence of a given male (Figure 2.5). $\beta$-values indicate that the effect of female 'overall sociality' was stronger than the effect of 'individual preference'.

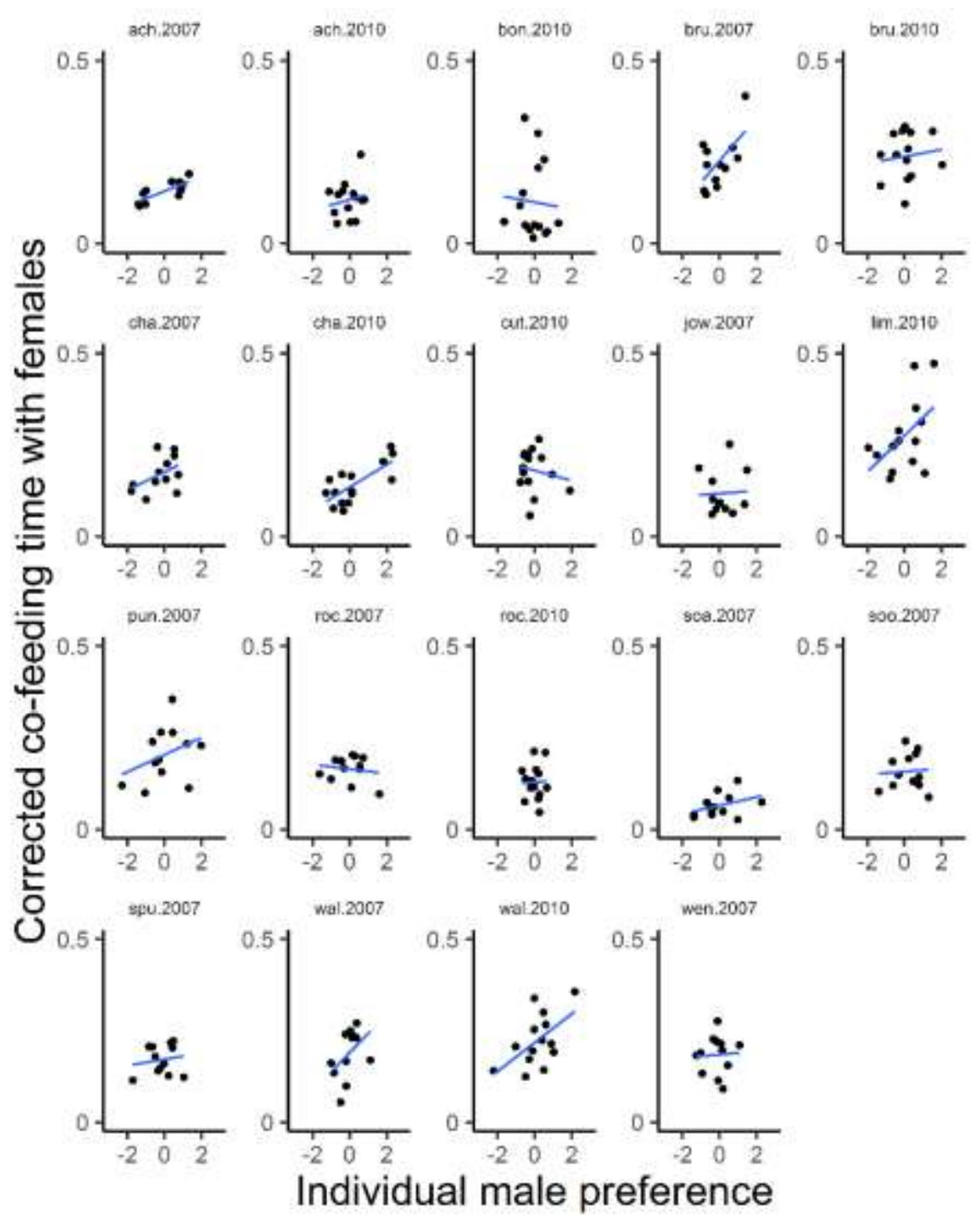

Figure 2.4: Male tolerance towards females in the context of feeding predicted by the strength of their affiliative relationship. The $x$-axis shows transformed 'individual preference' of each male subject and the $y$-axis the corrected co-feeding time of each male with different females. The blue lines indicate the linear regression predicted by the model. Above each graph the male ID and observation period is given. 


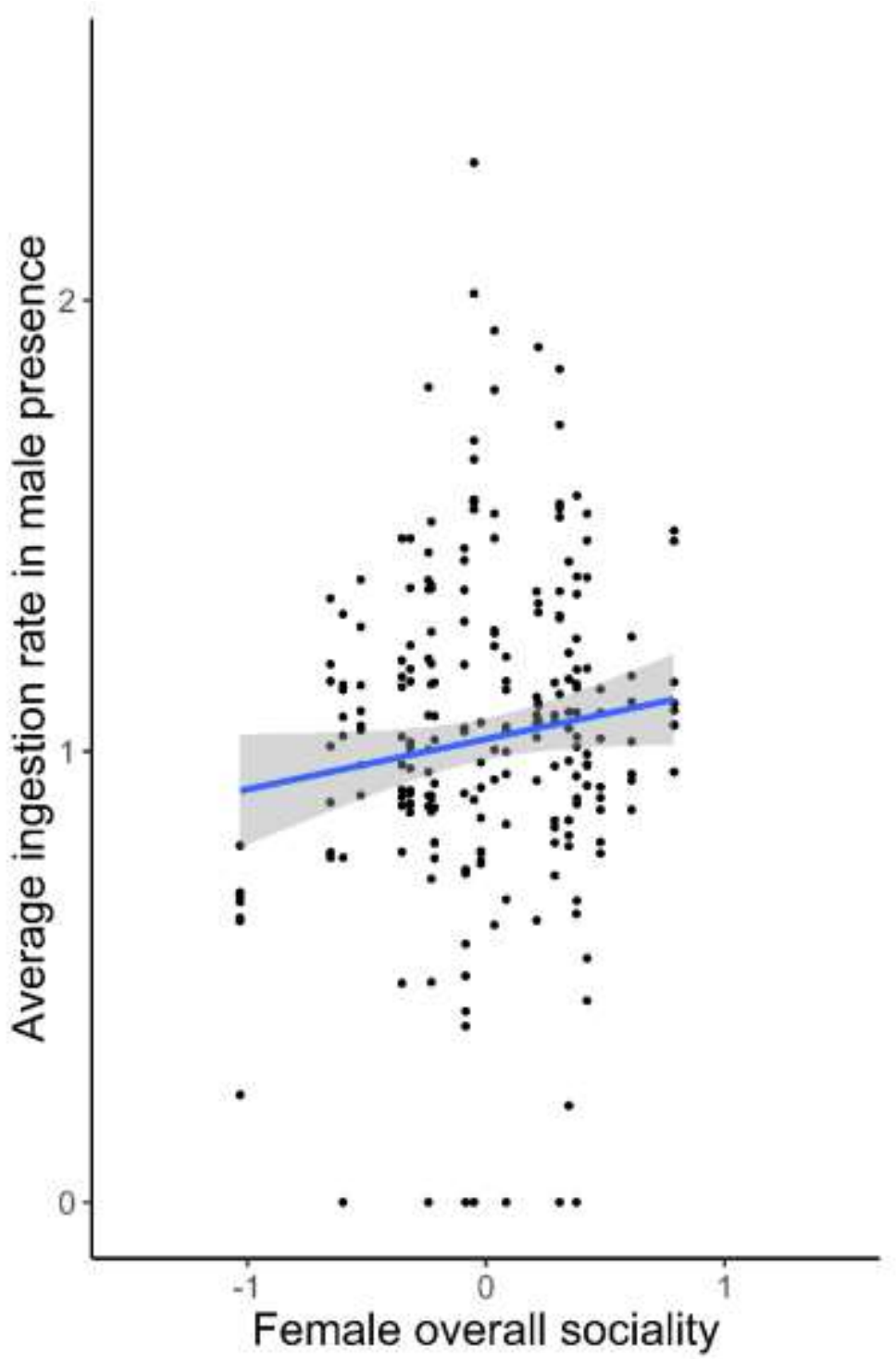

Figure 2.5: Average female ingestion rate in the presence of males predicted by the female's average CSI over all males. The $x$-axis shows transformed CSI values, the $y$-axis the average standardized ingestion rates of females. The blue line indicates the linear regression predicted by the model, with the grey shade as standard error. 


\section{DISCUSSION}

Our results are consistent with the idea that the formation of social bonds with males provides benefits to a female in form of reduced harassment, increased tolerance in the feeding context, increased agonistic support and increased food intake rates in the presence of closely bonded male partners. Female Assamese macaques seem to derive high stake benefits from males that should directly affect their reproductive success.

Reduced aggressiveness is often used to define the nature of affiliative relationships in primates (Pereira and McGlynn, 1997; Ostner and Kappeler, 1999; Crockford et al., 2013; Wittig et al., 2014). Yet, reduced aggression may also be regarded as a benefit of social bonding, particularly when aggression can incur serious costs for the recipient. In male-female relationships the amount of agonism is often driven by the amount of time a dyad spends together (Smuts, 1985). Thus, females face a constant trade-off between the benefits of spatial association (Lehmann and Boesch, 2009), and the costs of receiving more aggression, which can be reduced by forming an affiliative relationship with a male.

Agonistic support in within-group conflicts has been reported in various mammalian species, from dolphins (Connor et al., 2001; Connor, 2007) and terrestrial carnivores (e.g. Zabel et al., 1992; Villiers et al., 2003; Romero and Aureli, 2008; Smith et al., 2010), to primates (e.g. Noë and Sluijter, 1995; Watts, 1998; Widdig et al.,. 2000; Silk et al., 2004; Berghänel et al., 2010; Schülke et al., 2010; Kulik et al., 2011). Coalitions with same-sex partners are often considered to be a mutually beneficial means whereby participants can sustain or improve their social status (Wrangham, 1980; Zabel et al., 1992; Widdig et al., 2000; Villiers et al., 2003; Schülke et al., 2010; Smith et al., 2010). In opposite-sex relationships, coalitions are generally a response to harassment by higher ranking individuals (Seyfarth, 1978a; Smuts, 1985; Lemasson et al., 2008; Moscovice et al., 2010), and male-female rank-changing coalitions between unrelated individuals are rare (but see Surbeck et al., 2011 for mother-son coalitions in bonobos).

In Assamese macaques, males intervene in dyadic female-female conflicts less often than they do in female-male conflicts ( $20 \%$ versus $80 \%$ of agonistic support recorded), and which of the two females receives the support is less predictable than it is in male-female agonistic interactions. Hence, it is unlikely that male aid helps females improve or sustain their dominance rank. It is also unlikely that male-female coalitions do so for males. First, in the observed encounters, females did not influence the outcome of the interaction, i.e. conflicts were decided based on male dominance relations alone. Second, the directional consistency of male dominance relations was high already, if measured from dyadic male-male conflicts alone (see Table 2.2). It seems unlikely, therefore, that males gained status from supporting females against males. Male agonistic support rather served to protect the female from male 62 
harassment. In support of this, two-thirds of male aid occurred during the birth season, when females and dependent offspring are most vulnerable, but males have no need to defend females as sexual partners. Male-female association in the mating season predicts male mating success as well as male-infant association during the following non-mating season (Ostner et al., 2013), indicating some degree of male knowledge of paternity.

Nevertheless, a male who provided agonistic support risks severe costs through injuries, and should be sensitive to these. Our results show that the top three most dominant males provided $75 \%$ of the support, and almost all aggression (98\%) was directed to lower ranking individuals. The strategy to attack only lower ranking individuals (or alternatively support higher ranking individuals) lowers the risk of injury (Cheney and Seyfarth, 1990) and is quite common among primates (Bernstein and Ehardt, 1985; Chapais et al., 1991; Watts, 2002; Wittig et al., 2007; Borgeaud and Bshary, 2015) as well as hyenas (Engh et al., 2005; Smith et al., 2010). Paternity success in Assamese macaques is correlated with male dominance rank (Sukmak et al., 2014). Nevertheless, paternity alone or, rather, defence of biological offspring, cannot explain our results, as both mothers and non-mothers were supported by bonded males based on the strength of their relationship.

Female Assamese macaques were able to increase feeding rates in the presence of a bonded male. In female olive baboons, low level aggression, such as supplants from other females, is the best predictor of decreasing feeding rates (Barton, 1993). Our results show, that the stronger a female's affiliative relationship with a male, the less aggression she has to fear from him. The observation that increased sociability can reduce vigilance and improve foraging (Josephs et al., 2016), makes possible that a female may be less vigilant when feeding with that male and increase her feeding rates. Second, the strength of their affiliative relationship also predicts the male's willingness to support that female. Consequently, the female can count on the male's support when being harassed by other males. The combination of fearing less aggression by a male and building on his support might enable a female to be even less vigilant and concentrate more on feeding while the particular male is around. The reduced aggressiveness within the relationship and the male's disposition to support a female therefore work together to increase a female's feeding rates. Nevertheless, our results show that the effect of the relationship strength with a male on the ingestion rates of females is very low. This may be due to a great number of ecological factors we could not consider in our model. For once, not only the male in presence should affect female feeding but also each other member in the feeding group. Testing this would need more detailed information on the distance to everyone in the food patch as well as each individual's activity at the given time. Future studies should measure female vigilance in the presence or absence of bonded males, with regards to the distance and relationships to other co-feeders as well, more directly to test this point formally. 
A social bond with a male can positively affect a female's lifetime reproductive output in at least three ways. First, fewer injuries might improve her own survival prospects. Second, reduced harassment increases food intake rates, and can shorten interbirth intervals. Third, increased food intake enhances her maternal investment and in turn her offspring's survival. The evolution of social bonds with particular males may not only enable females to reduce the costs of increased feeding competition inflicted on them by associating with multiple males, but even to turn them into adaptive benefits. Although it has been established that overall malefemale sociality can be associated with increased longevity (Archie et al., 2014), it remains to be shown that opposite sex social bonds yield similar effects. Where males are in short supply, females should compete to affiliate with those males (Palombit et al., 2001). Future studies should therefore tackle the question of female competition for male partners, and whether males in general or certain male traits attract female attention. 


\section{ACKNOWLEDGEMENTS}

We thank the National Research Council of Thailand (NRCT) and the Department of National Parks, Wildlife and Plant Conservation (DNP) for the permission to conduct this study and for all the support (permit 0004.3/3618, 0002.3/2647, 0002/17, 0002/2424). We are grateful to Kanjana Nitaya, Kitti Kreetiyutanont, Mongkul Kumsuk, Jarupol Prabnasuk and Thanee Wongnak (Phu Khieo Wildlife Sanctuary). We thank A. Koenig and C. Borries, who developed the field site at Huai Mai Sot Yai. Special thanks goes to D. Bootros, N. Bualeng, A. Chunchaen, A. Ebenau, I. Fürtbauer, R. Intalo, S. Jomlotwong, N. Juntuch, J. Kalbitz, M. Karlstetter, T. Kilawit, S. Macdonald, W. Nueorngshiyos, D. Pesek, N. Ponganan, S. Rogahn, P. Saaisawasthikul, K. Srithorn, M. Swagemakers, J. Wanart, B. Whitman, and T. Wisate for their excellent help in the field, and to Andreas Berghänel, Anja Ebenau, Josefine Kalbitz and Sally Macdonald for their support. We thank Roger Mundry for the permission to use statistical packages developed by himself. Finally, we want to thank Pat Backwell, Peter Henzi and anonymous reviewers for their very constructive comments on previous drafts of our manuscript. This research was supported by the Max-Planck Society, and the German Initiative of Excellence through funds to University of Göttingen and the Studienstiftung des Deutschen Volkes to $\mathrm{CBH}$. 


\title{
Chapter 3
}

\section{Female competition for 'power' and 'commitment' in male partners - Assamese macaques as a test case}

\author{
Christine B. Haunhorst ${ }^{a}$, Michael Heistermann ${ }^{c}$, Ines Fürtbauer ${ }^{d}$, \\ Oliver Schülke $^{\mathrm{a}, \mathrm{b}}$ \& Julia Ostner ${ }^{\mathrm{a}, \mathrm{b}}$
}

a Department of Behavioral Ecology, Johann-Friedrich-Blumenbach Institute for Zoology and Anthropology, Georg August University Göttingen, Germany

${ }^{\mathrm{b}}$ Research Group Primate Social Evolution, German Primate Centre, Göttingen

${ }^{c}$ Endocrinology Lab, German Primate Centre, Göttingen

${ }^{d}$ Department of Biosciences, College of Science, Swansea University

Journal of Human Evolution, to be submitted 


\begin{abstract}
Studies on female mammals competing for male partners mostly investigate direct mating competition. If males can enhance females' access to resources, males become a valuable resource over which females may compete. While female competition for a male partner outside the mating context is well studied in humans, evidence from non-human primates is scarce. We studied two groups of wild Assamese macaques (Macaca assamensis), a species where females gain benefits from bonding with particular males. We used behavioral data as well as fecal glucocorticoid analysis to investigate what behaviors females deploy to compete over males, whether a female's success in competition over a male was reflected in fecal glucocorticoid metabolite levels, and which male traits were most attractive for females. We found assortative bonding by rank between females and males which together with females being responsible for initiating and maintaining contact suggests direct female competition over males. Outcompeting other females that shared the same top partner resulted in elevated fecal glucocorticoid metabolite levels, indicating that females may face a trade-off between the costs and benefits involved in successful competition. Two male traits independently predicted male attractiveness: (1) current dominance rank, a measure of male ability to provide access to resources, and (2) former affiliation with immatures, a measure of paternal quality. Our results suggest that female Assamese macaques compete with some costs for access to male partners to gain benefits in form of protection and paternal care.
\end{abstract}

Keywords: Female competition, assortative bonding, female investment, male traits, dominance rank, paternal care, fecal glucocorticoid metabolites 


\section{INTRODUCTION}

The formation of male-female bonds is a core element of human societies (Alexander and Noonan, 1979; Chapais, 2008). Male provisioning of females during energetically demanding phases, the development of paternal care, and the division of labor are all implicated in the evolution of the male-female bond in humans (Alexander and Noonan, 1979; Lovejoy, 1981; Marlowe, 2000; Hawkes, 2004; Quinlan and Quinlan, 2007, 2008). The precise evolutionary pathways and causal relationships between these factors are still being debated but there is broad agreement that they have favored the evolution of large brains, elaborate cognitive abilities, and the unparalleled ecological success of humans (Kaplan et al., 2000, 2009; Chapais, 2013; Coxworth et al., 2015; Fletcher et al., 2015). If male behavioral traits (e.g. provisioning, paternal care) enhance offspring fitness, females should choose males based on those traits to increase their reproductive output. If males featuring desirable traits are scarce, females should compete for male partners.

Sexual selection theory was long interpreted in terms of female mammals exclusively competing for resources, and males primarily competing for access to female mating partners (Trivers, 1972; Emlen and Oring, 1977; Clutton-Brock and Harvey, 1978; Clutton-Brock, 1989; Tobias et al., 2012). Whereas evidence for female competition over access to mates is accumulating (Buss, 1988; Bro-Jørgensen, 2002; Rosvall, 2011; Stockley and Bro-Jørgensen, 2011; Stockley and Campbell, 2013), less is known about female competition for long-term bonded partners (or 'friends': Silk, 2002) based on resources or 'services' offered. Reproductive success in female mammals is constrained by the availability of energy resources to sustain high energetic demands during gestation and lactation (Sadleir, 1969; Bongaarts, 1980; Schneider, 2004) as well as individual differences in infant mortality (CluttonBrock, 1988), leading females often to compete directly for food (Emlen and Oring, 1977; Wrangham, 1980; Sterck et al., 1997; Silk, 2007; Stockley and Bro-Jørgensen, 2011; Pusey and Schroepfer-Walker, 2013) or other resources essential to reproductive performance, such as nest sites, or safety (van Schaik, 1989; Dammhahn and Kappeler, 2009). If males provide resources to females and vary in quantity or quality of resources provided (e.g. access to food: Haunhorst et al., 2017; protection against infanticide: Borries et al., 1999; Broom et al., 2004; Engh et al., 2006; Hawkes, 2004; Opie et al., 2013; Palombit, 2009; Paul et al., 2000; van Schaik and Kappeler, 1997) bonding with particular males could be an indirect manifestation of resource competition (Campbell, 2004; Emlen and Oring, 1977; Stockley and BroJørgensen, 2011). In this case, females should select males based on their qualities.

Physical superiority of higher-ranking males may improve a male's ability to successfully protect offspring and to enhance access to energy resources (Hamilton and 
Bulger, 1990; Watts, 2010). Females may increase their reproductive success primarily by bonding with high-ranking males or males that have provided paternal care in the past (Fernández-Duque et al., 2009). In modern human societies females compete for 'power', i.e. being of high social status and having access to valuable resources, or 'commitment', i.e. loyalty towards female and offspring in male partners (Campbell, 2004). This form of female competition can involve high physical and psychological costs (Faer et al., 2005). Hamadryas baboons (Papio hamadryas) living in one-male units show signs of female competition for leader males, as female-male grooming time is a function of female dominance rank (Colmenares et al., 2002). By grooming, females reduce the threat of aggression from the leader male, enhance access to resources and gain protection against harassment by other group members (Colmenares et al., 2002). In chacma baboons (Papio ursinus) living in multimale groups females only compete for access to the male that most likely sired their offspring who is known to provide protection from other potentially infanticidal males (Palombit et al., 2001). These relationship break up upon the infant's death (Palombit et al., 1997). Only few other studies identified female competition for males outside the mating context in femalebonded non-human primate groups (Smuts, 1985; Lemasson et al., 2008; Archie et al., 2014).

We tested whether Assamese macaque females living in multimale - multifemale groups compete for access to males, which male qualities they compete for and whether success in competition is reflected in glucocorticoid metabolite levels. In doing so we broaden the comparative data on the social evolution of humans.

Assamese macaques form stable social bonds with males which are not equally distributed across all males (Haunhorst et al., 2016), and last, despite a promiscuous mating system (Fürtbauer et al., 2011a), across reproductive seasons and several years (Haunhorst et al., 2016). Male-female association in the mating season is predictive of future male-infant association which in turn predicts male agonistic support for this infant (Ostner et al., 2013; Minge et al., 2016), indicating some degree of male care. Apart from male care for their offspring, females benefit from bonding with a male in form of male agonistic support and higher food intake rates in the male's presence (Haunhorst et al., 2017). Above and beyond the preferential agonistic support for closely bonded female partners, the frequency of male support to females is predicted by male dominance rank (Haunhorst et al., 2017). Hence based on the strength of opposite sex dyadic affiliative relationships male Assamese macaques provide resources for females that may enhance a female's reproductive success.

We tested a number of predictions regarding female competition for males: First, we tested the occurrence of competition for male partners. Since dominance hierarchy is a predictor for access to resources (or access to male partners, e.g.: Colmenares et al., 2002; Palombit et al., 2001), female dominance rank should predict relationship strength to high quality males (1a; Haunhorst et al., 2017). It was previously shown, that males of all dominance ranks may be top partners of more than one female (Haunhorst et al., 2016). We predicted 
that when two or more females share the same top partner (i.e. a 'competitive situation'), the higher ranking a female is the stronger her relationship to the male (1b). We further investigated whether females reacted to competitive situations with changes in glucocorticoid metabolite levels if failing to either build the strongest bond or spend the most time with the male. We predicted that females' success in a competitive situation for a male partner to be negatively associated with fecal glucocorticoid metabolite levels (2). Third, we predicted that the strength of a male's affiliative relationships with females increases with his power measured as his dominance rank and with the time spent affiliating with immatures in the past birth season as proxy for paternal qualities (3).

\section{METHODS}

\section{Study site and population}

We conducted our study in Phu Khieo Wildlife Sanctuary (PKWS; $>1,600$ km², 16 $6^{\circ}-35^{\prime} \mathrm{N}$, $101^{\circ} 20^{\prime}-55^{\prime}$ E, 300-1,300m) in north-eastern Thailand (Schülke et al., 2011). The study area is covered by hill evergreen forest, dry evergreen forest, dry dipterocarp, and bamboo stands (Borries et al., 2002). PKWS is part of an interconnected system of eight protected areas, the 6500sqkm Western Isaan Forest Complex (Grassman et al., 2005) in Chaiyaphum and harbors a diverse community of large mammals and predators indicating low levels of habitat disturbance.

The study population consisted of two wild groups of fully habituated Assamese macaques. Assamese macaques are seasonal breeders (Fürtbauer et al., 2010), with a mating season (ms) from October through January and a non-mating season (nms) from February to September. Most infants are born between April and June (Fürtbauer et al., 2010). At any time, both groups included several adult males, several adult females and a large number of immatures (Table 3.1). 
Table 3.1: Average focal animal observation hours per season and focal animal sex [mean \pm SD, hrs], and number of observed adult individuals. AS and $A O$ refer to the two study groups.

\begin{tabular}{|c|c|c|c|c|c|}
\hline - & & mean $\pm \mathrm{S}[$ & ration [hrs] & $\begin{array}{l}\text { Number of ad } \\
\text { animals }\end{array}$ & tocal \\
\hline Observation period & Season & Male & Female & Male & Female \\
\hline Oct 2009 - Jan 2010 & $\mathrm{~ms}$ & $49.1 \pm 7.8$ & no obs. & 10 & 15 \\
\hline Feb 2010 - Sep 2010 & $\mathrm{nms}$ & $49.4 \pm 21.4$ & $49.7 \pm 5.3$ & 10 & 15 \\
\hline Oct 2010 - Jan 2011 & $\mathrm{~ms}$ & $49.4 \pm 6.0$ & $39.0 \pm 4.5$ & 10 & 15 \\
\hline Feb 2011 - May 2011 & $\mathrm{nms}$ & $15.3 \pm 1.7$ & $24.3 \pm 3.3$ & 10 & 15 \\
\hline Oct 2012 - Jan 2013 & $\mathrm{~ms}$ & $19.6 \pm 5.9$ & no obs. & AS: 7; AO: 10 & $\begin{array}{l}\text { AS: } 11 ; A O: \\
11\end{array}$ \\
\hline Feb 2013 - Sep 2013 & $\mathrm{nms}$ & $81.1 \pm 10.4$ & $46.5 \pm 3.0$ & AS: 7; AO: 10 & $\begin{array}{l}\text { AS: } 10 ; A O: \\
12\end{array}$ \\
\hline Oct 2013 - Jan 2014 & $\mathrm{~ms}$ & $53.5 \pm 12.6$ & $27.8 \pm 5.9$ & AS: 8; AO: 10 & $\begin{array}{l}\text { AS: } 11 ; A O: \\
12\end{array}$ \\
\hline Feb 2014 - Sep 2014 & $\mathrm{nms}$ & $82.8 \pm 18.4$ & $19.4 \pm 2.4$ & AS: $7 ;$ AO: 6 & $\begin{array}{l}\text { AS: } 11 ; A O: \\
10\end{array}$ \\
\hline
\end{tabular}

\section{Behavioral data collection}

On approximately $N=20$ days per month, we followed the two study groups (AS, AO) from dawn to dusk. We observed adult males and females in $30 \mathrm{~min}$ focal animal protocols using continuous and instantaneous recording (Table 3.1; Altmann, 1974). We used 6 twohour time-blocks between 06:00 am and 06:00 pm to distribute focal observations evenly across individuals. We observed males in group AS from 2009 through 2014, and included group AO from 2012 onwards. We collected data on females in two non-consecutive periods (2010/11, 2013/14).

In a total of 7757 hours of focal animal protocols we recorded the frequency and duration of three affiliative behaviors: (i) close proximity $(<1.5 \mathrm{~m})$, (ii) body contact, and (iii) grooming in the continuous protocol. When one individual approached another within at least $1.5 \mathrm{~m}$ and stayed in this distance for at least $10 \mathrm{sec}$, we defined an approach into close proximity. We recorded a departure when one of the individuals left the $1.5 \mathrm{~m}$ proximity of the other individual. We recorded body contact when two individuals were standing, sitting or lying so close to each other that part of their bodies touched. We defined an interaction in close proximity or body contact whenever one individual started the behavior, and the time spent performing a behavior as the total duration of the interaction. We recorded grooming when one individual manipulated with its fingers the fur of another individual, removing dirt or parasites. We defined a grooming interaction as one continuous event of one individual grooming another 
that was not interrupted by more than $10 \mathrm{sec}$ of pausing, the performance of other behaviors, or a change in the actor and recipient roles. Additionally, we recorded all agonistic interactions within the continuous protocol. We defined agonism as one individual showing aggressive (lunge, chase, slap, push-and-pull, bite, ground slap, open mouth) or submissive (make room, silent bared teeth, flee, crouch) behavior towards another (see Ostner et al., 2008). Additionally, agonistic interactions were recorded ad libitum. Every $10 \mathrm{~min}$, we recorded all individuals in the focal animal's $5 \mathrm{~m}$ proximity.

\section{Behavioral data analysis}

We chose the dyadic composite sociality index (CSI: Haunhorst et al., 2016; Silk et al., 2006a) as a measure of the strength of the affiliative relationship between a male and a female relative to the group's average across all male-female relationships for a given group and period (ms vs. nms of a given year). For each dyad, we included the total duration (time spent performing a behavior) and frequency (number of interactions) of three behaviors: being in close proximity $(<1.5 \mathrm{~m})$, body contact, and grooming. We subtracted the duration of grooming from the duration of body contact and the duration of body contact from the duration of close proximity, as those behaviors are nested into each other. We controlled for biases due to varying observation times by dividing the behaviors by the total observation time of the dyad. To standardize on the level of the social group, we divided each resulting behavior by the average across all dyads in the group in a given period. We calculated the index as follows:

$$
C S I_{x y}=\frac{\sum_{i=1}^{b}\left(\frac{f_{i x y}}{f_{i}}+\frac{d_{i x y}}{d_{i}}\right)}{2 b}
$$

Here $b$ is the number of behaviors that contribute to the index, $f_{i x y}$ is the frequency of behavior $i$ for the dyad $x y, f_{i}$ is the mean of the frequency of behavior $i$ across all male-female dyads, $d_{i x y}$ is the total duration of behavior $i$ for the dyad $x y$, and $d_{i}$ is the mean of the total duration of behavior $i$ across all male-female dyads. The index has a minimum of 0 , a mean of 1 and increases with the strength of the dyadic affiliative relationship (Silk et al., 2013). We ran rowwise matrix correlations in R (version 3.1.2, R Core Team 2014) for all combinations of the 6 behavioral measures and found components of the CSI to be significantly positively correlated (15 correlations; all $p<0.001$; range of average, row-wise Spearman's rho $0.47-0.98$; mean \pm $\mathrm{SD}$ row-wise average rho $=0.70 \pm 0.14$ ).

To compute a dominance hierarchy we used all dyadic agonistic interactions where we observed clear submissive signs (make room, silent bared teeth, unprovoked give ground; Ostner et al., 2008) from both continuous focal protocols and ad libitum data. We calculated a hierarchy separately for males and females, based on the normalized David's Score (Schmid and De Vries, 2013) for each period of data collection (Table 3.2). In the following analyses we 
used standardized dominance rank (ranging from 0 to 1 ) to control for the number of individuals in the group and sex. The standardized dominance rank translates into the highest-ranking individual as 1 and the lowest ranking individual as 0 , and other individuals distributed evenly in between. This approach allows for comparison of dominance hierarchies of varying sizes. We calculated the similarity or difference in dominance rank between males and females as absolute value of male standardized dominance rank minus female standardized dominance rank. Difference in dominance rank could vary between 0 and 1 , with 0 indicating no difference in dominance rank (i.e. highest ranking male with highest ranking female, or lowest ranking male with lowest ranking female) and 1 with the highest possible difference in dominance rank (i.e. highest ranking male with lowest ranking female, or vice versa).

Table 2: Details on dominance hierarchies with mean and standard deviation (mean \pm SD) across observation periods and groups per sex.

\begin{tabular}{|l|l|l|l|l|l|l|l|}
\hline & $\begin{array}{l}\text { Number } \\
\text { of dyads }\end{array}$ & $\begin{array}{l}\text { Number of } \\
\text { conflicts }\end{array}$ & $\begin{array}{l}\text { Linearity } \\
\text { index h' }\end{array}$ & $\begin{array}{l}\text { Unknown } \\
\text { relationships } \\
(\%)\end{array}$ & $\begin{array}{l}\text { Directional } \\
\text { consistency }\end{array}$ & $\begin{array}{l}\text { Two-way } \\
\text { relationships } \\
(\%)\end{array}$ & Ties (\%) \\
\hline Male & $37.3 \pm 13.9$ & $232.3 \pm 52.1$ & $1 \pm 0$ & $8.27 \pm 5.97$ & $0.96 \pm 0.02$ & $10.0 \pm 5.8$ & $1.53 \pm 1.16$ \\
\hline Female & $79.3 \pm 20.4$ & $453.7 \pm 82.3$ & $0.82 \pm 0.06$ & $10.6 \pm 1.36$ & $0.98 \pm 0.02$ & $4.4 \pm 3.1$ & $0.33 \pm 0.47$ \\
\hline
\end{tabular}

To assess male "attractiveness" to females, we ranked, for each female, all males by their CSI value from a female's perspective. We standardized these values like the dominance ranks by assigning a value of 1 to the strongest and a value of 0 to the weakest relationships and spreading all others equally in between. From these values, we calculated an average CSI rank across all females for each male (CSI-rank hereafter). The highest CSI-rank indicates the highest "attractiveness" to females.

To assess a male's affiliation with immatures, as a proxy for paternal quality, we computed a male-immature affiliation index by adding the time spent in three affiliative behaviors (close proximity, body contact, and grooming) with all immatures below three years of age (mean \pm SD: $21.3 \pm 3.5$ ) for each male. We did not include immatures older than 3 years to avoid confusion with other motivations for affiliation than paternal care since age at first birth for female Assamese macaques is five (Fürtbauer et al., 2010). We included the total time of each affiliative behavior without subtraction from each other (unlike the calculation of the CSI), thereby weighting time spent in body contact over close proximity and grooming over the other two behaviors which results in males grooming immatures being scored as more social than males that spend the same time in proximity to immatures but never groomed with them. We standardized the sum of the three behaviors on the level of the respective period across all 74 
males in the group by dividing a male's value by the average value of the group in the respective period, to eliminate the effect of the number of resident males and immatures present at times and seasonal dependent behavioral changes. The standardized index varies between 0 and $\infty$, with high values indicating males spending increasing amounts of time affiliating with immatures.

\section{Fecal sample collection and glucocorticoid (fGC) analysis}

We collected fecal samples of all adult females opportunistically during both reproductive seasons (nms 2010, ms 2010/11, ms 2013/14 and nms 2014; $n=950$, mean \pm SD per female and season: $13.2 \pm 2.96$ ) to assess female glucocorticoid metabolite levels. We collected samples uncontaminated with urine or water immediately after defecation. We extracted samples at the field site by transferring approximately $0.5 \mathrm{~g}$ (exact mass recorded) of fresh fecal material into $4 \mathrm{ml}$ of $80 \%$ watery ethanol and processed them using a validated field extraction protocol (Shutt et al., 2012). Two $\mathrm{ml}$ of each sample extract were stored at ambient temperatures for a maximum of 9 months (Shutt et al., 2012) until transport to the endocrinology laboratory at DPZ where all samples were stored frozen at $-20^{\circ} \mathrm{C}$ until hormone analysis.

All fecal extracts were analyzed for immunoreactive $11 \beta$-hydroxyetiocholanolone, which is a major metabolite of cortisol in the feces of primates (Heistermann et al., 2006), including the Assamese macaque (Ostner et al., 2008). The measurement of $11 \beta$ hydroxyetiocholanolone has been validated and successfully applied for monitoring adrenocortical activity in numerous primate species of all major taxa (Heistermann et al., 2006; Weingrill et al., 2011; Shutt et al., 2012; Rimbach et al., 2013; Hämäläinen et al., 2014). It was also previously successfully used to assess faecal glucocorticoid metabolite levels in both male and female Assamese macaques (Ostner et al., 2008; Fürtbauer et al., 2014). The assay was carried out as described in detail by Heistermann et al. (2004). Prior to analysis, we diluted fecal extracts 1:200 to 1:2000 (depending on concentration) with assay buffer, to bring hormone concentrations into the working range of the assay. Sensitivity of the assays at $90 \%$ binding was $2.0 \mathrm{pg}$. We determined intra- and inter-assay coefficients of variation (CV) by replicate measurements of high- and low-value quality controls for 2010/11 and 2013/14, separately. In each of the two study periods, intra- and inter-assay CV values were $<10 \%$ and $<15 \%$, respectively.

\section{Statistical analysis}

General procedure

We fitted all models in R (version 3.2.2; R Core Team 2014) using the functions 'Im', 'Imer' and 'glmer' of the R-package 'Ime4' (Bates et al., 2014). In the case of Linear mixed models with Gaussian error link function and Generalized linear mixed models with binomial error link 
function (LMM and GLMM, respectively; Baayen, 2008) we followed the procedure as follows. Prior to analysis, we transformed variables if necessary to achieve an approximately normal distribution of residuals (reported in detail below). We z-transformed all predictors and fixed effects (to a mean of zero and a standard deviation of one), hence all estimates reported are standardized betas. We checked for whether the assumptions of normally distributed and homogeneous residuals were fulfilled by visually inspecting $Q-Q$ plots and the residuals plotted against fitted values. We checked for model stability by excluding subjects one at a time from the data (functions provided by Roger Mundry, Leipzig). To rule out collinearity of included factors, we derived Variance Inflation Factors (VIF, Field, 2005) using the function 'vif' of the R-package 'car' (Fox and Weisberg, 2011) applied to a standard linear model excluding the random effects. We found no obvious influential cases, nor obvious deviations from the assumptions of normality and homogeneity of residuals (Field, 2005; Forstmeier and Schielzeth, 2011). We established the significance of the full model as compared to the null model (comprising only fixed control and random effects) using a likelihood ratio test ( $R$ function 'ANOVA' with argument test set to "Chisq"; Dobson, 2002; Forstmeier and Schielzeth, 2011). To allow for a likelihood ratio test we fitted the models using Maximum Likelihood (rather than Restricted Maximum Likelihood; Bolker et al., 2009). P-values for the individual effects were based on likelihood ratio tests comparing the full with respective reduced models (Barr et al., 2014; $\mathrm{R}$ function drop1). We found all full models reported in the results to be different from the respective null model (Table 3.3).

For all models we calculated the 'conditional' $R^{2}$, a measure for how well the model fits the data. It represents the variance of the results explained by the model (i.e., fixed control and random effects) using the function "MuMln".

\section{Assortative bonding}

To test the prediction of assortative bonding (1a) we used a LMM including dominance rank difference (diff.rank) as predictor. We added male dominance rank, female dominance rank and reproductive season ( $\mathrm{ms} / \mathrm{nms}$ ) as fixed effects to control for potential behavioral changes associated with dominance rank or the respective seasons. We included the dyadic, male and female identification, as well as group ID (AS vs. AO) and year as random effects. We powertransformed CSI by 0.3 and dominance rank similarity by 0.5 prior to analysis to achieve approximately symmetric distribution and avoid influential cases. Male and female dominance rank both were approximately symmetrically distributed.

\section{Female competition}

We further established the occurrence of female competition for males by evaluating the situation from each male's perspective, considering only those females that did share the same male as top partner (highest CSI; Haunhorst et al., 2016). We considered it to be a competitive 
situation if two or more females shared the same male as top partner (1b). As competition is mostly settled by dominance hierarchy, with the higher ranking individual having priority in gaining access to resources (Whitten, 1983; van Noordwijk and van Schaik, 1987; Barton, 1993; Barton and Whiten, 1993), we expected that the higher the dominance rank of a female the higher ranking the affiliative relationship with a male compared to other females. Hence, we ranked females within a competitive situation by 1) the strength of their relationship with the male from the male's perspective and 2) by female dominance rank. In case of 5 females "competing" for the same male, i.e. sharing the same male as top partner, these females were sorted 1 to 5 in both relationship strength and dominance rank, respectively. We ran a linear regression with the order of relationship strength as the response and the dominance rank within the competitive situation as a predictor.

\section{Glucocorticoid metabolite levels}

To assess whether females' glucocorticoid metabolite levels are associated to female competition for male partners (3), we ranked females within a competitive situation (i.e. highest value compared to competitors equals 1 ) by the fecal GC levels, the CSI with the respective male, the grooming effort put into the relationship and the time, they spent in the male's proximity $(1.5-5 \mathrm{~m})$. We ran a LMM with $\mathrm{fGC}$ as response and the CSI, time spent in proximity and grooming effort as main predictors. Additionally, we controlled for the effect of reproductive state and season. We included female ID, year ID and group ID (AO vs. AS) as random factors.

\section{Male attractiveness}

To assess the male qualities females might compete for, we ran a GLMM with the time affiliating with immatures (see above) and male dominance rank as main predictors and CSIrank as the response. We included the reproductive season (ms vs. nms) as a fixed factor because behavior might change due to changes in social and sexual interactions or the presence of infants (Fürtbauer et al., 2014; Haunhorst et al., 2016). We used male ID and group ID (AS vs. AO) as random factors to control for individual and group-specific differences. 


\section{RESULTS}

Table 3.3: Summary of GLMMs and LMMs with response, predictors and fixed control factors with comparison of full vs. null models.

\begin{tabular}{|c|c|c|c|c|c|c|}
\hline Model name & Response & Predictors & $\begin{array}{l}\text { Control } \\
\text { factors }\end{array}$ & $x^{2}$ & df & $p$ \\
\hline $\begin{array}{l}\text { Assortative } \\
\text { bonding }\end{array}$ & $\mathrm{CSI}$ & $\begin{array}{l}\text { Difference in } \\
\text { dominance } \\
\text { rank }\end{array}$ & $\begin{array}{l}\text { Female } \\
\text { dominance } \\
\text { rank; Male } \\
\text { dominance } \\
\text { rank; Season }\end{array}$ & 574.7 & 3 & $<0.001$ \\
\hline Stress & fGC rank & $\begin{array}{l}\text { CSI rank; } \\
\text { Proximity rank; } \\
\text { Female } \\
\text { grooming effort }\end{array}$ & $\begin{array}{l}\text { Female } \\
\text { reproductive } \\
\text { state; Season }\end{array}$ & 126.4 & 3 & $<0.001$ \\
\hline $\begin{array}{l}\text { Male } \\
\text { attractiveness }\end{array}$ & CSI rank & $\begin{array}{l}\text { Male } \\
\text { dominance } \\
\text { rank; Immature } \\
\text { affiliation }\end{array}$ & Season & 162.9 & 3 & $<0.001$ \\
\hline
\end{tabular}

\section{Assortative bonding}

Overall, we tested $N=835$ male-female dyads. We found that the closeness in dominance rank within a dyad had a strong negative effect on relationship strength (Figure 3.1). The closer a male and female were in dominance rank (e.g. both ranking very high), the stronger was the relationship between the two ( $\beta \pm \mathrm{SE}:-0.21 \pm 0.05, t=-4.10, p<0.001)$. Additionally, both male ( $\beta \pm$ SE: $0.33 \pm 0.08, t=4.40, p<0.001)$ and female $(\beta \pm$ SE: $0.17 \pm 0.06, t=2.95, p<0.005)$ dominance rank were positively associated with relationship strength. The higher ranking an individual, the stronger its dyadic relationships to an opposite-sex partner could be. In the nonmating season the relationship strength was lower than in the mating season ( $\beta \pm$ SE: $-0.08 \pm$ $0.02, t=-3.51, p<0.001)$. The complete model explained $28 \%$ of the variance in dominance rank similarity $\left(R^{2}=0.28\right)$. 


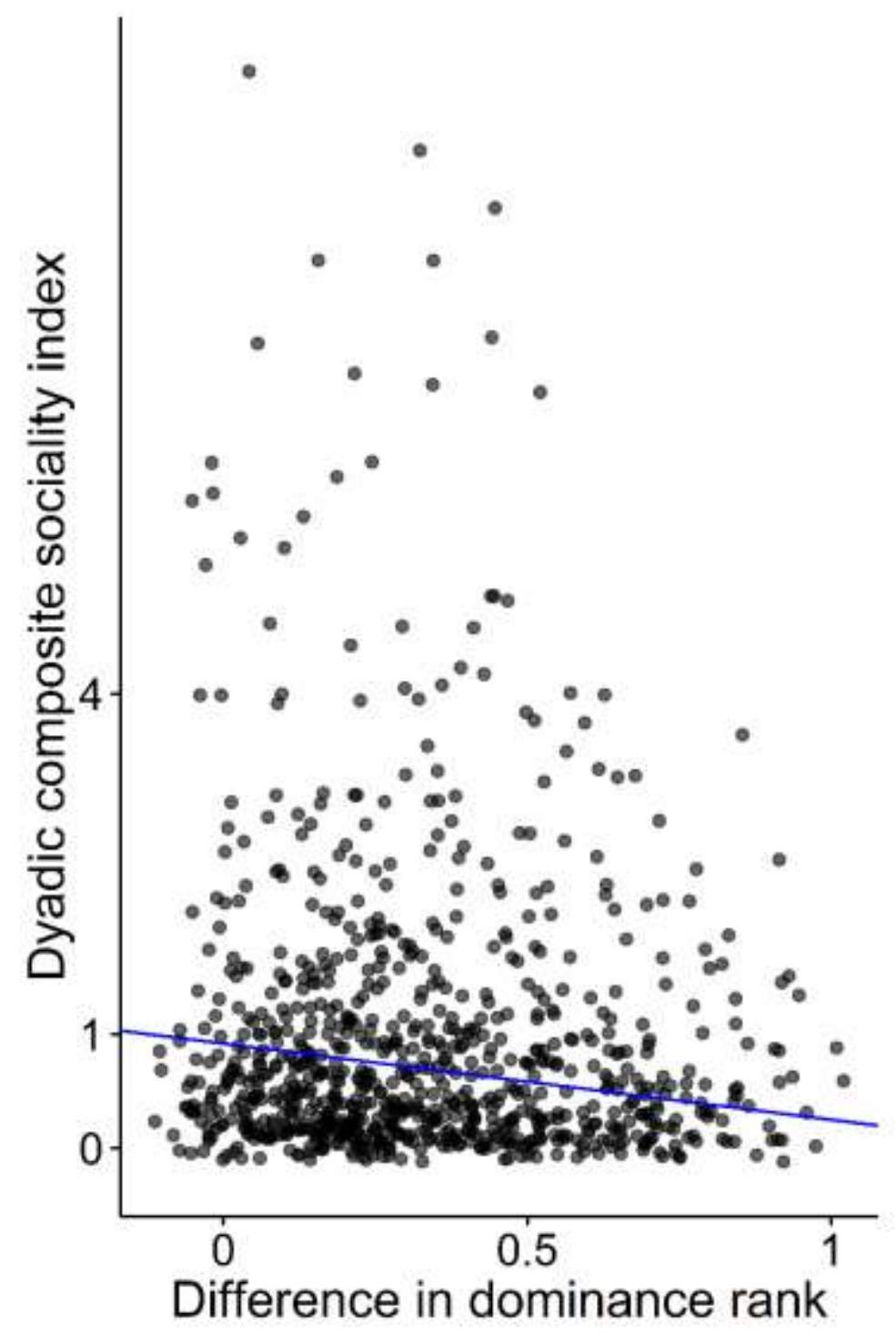

Figure 3.1: The strength of male-female relationships as predicted by the partners' difference in dominance rank. Male and female dominance ranks are measured on separate scales and a difference of zero indicates that the female occupies the same rank in the female dominance hierarchy as the male in the male dominance hierarchy. The blue line indicates the relationship between the difference in dominance rank and the strength of a relationship. Note that the LMM also controlled for effects of season, male and female dominance rank, which are not shown.

\section{Female competition}

The number of males in competitive situations $(N=26)$ varied widely across the study period with 2 to 6 males being top partner of 2 to 5 females, adding up to 70 data points. Males in competitive situations held all possible dominance ranks from alpha to the lowest ranking position (upper half of hierarchy: $N=18$, lower half: $N=8$ ). We found that the strength of a female's relationship compared to others in the same competitive situation was strongly associated with her rank in the female dominance hierarchy (Figure 3.2; estimate \pm SE $=0.49$ $\pm 0.10 ; z=5.13 ; p<0.001)$. In $50 \%$ of cases $(N=26)$, a female was ordered as highest in both categories. Only $11 \%$ of cases are found below the predicted regression line, showing that few cases ran counter the prediction. 


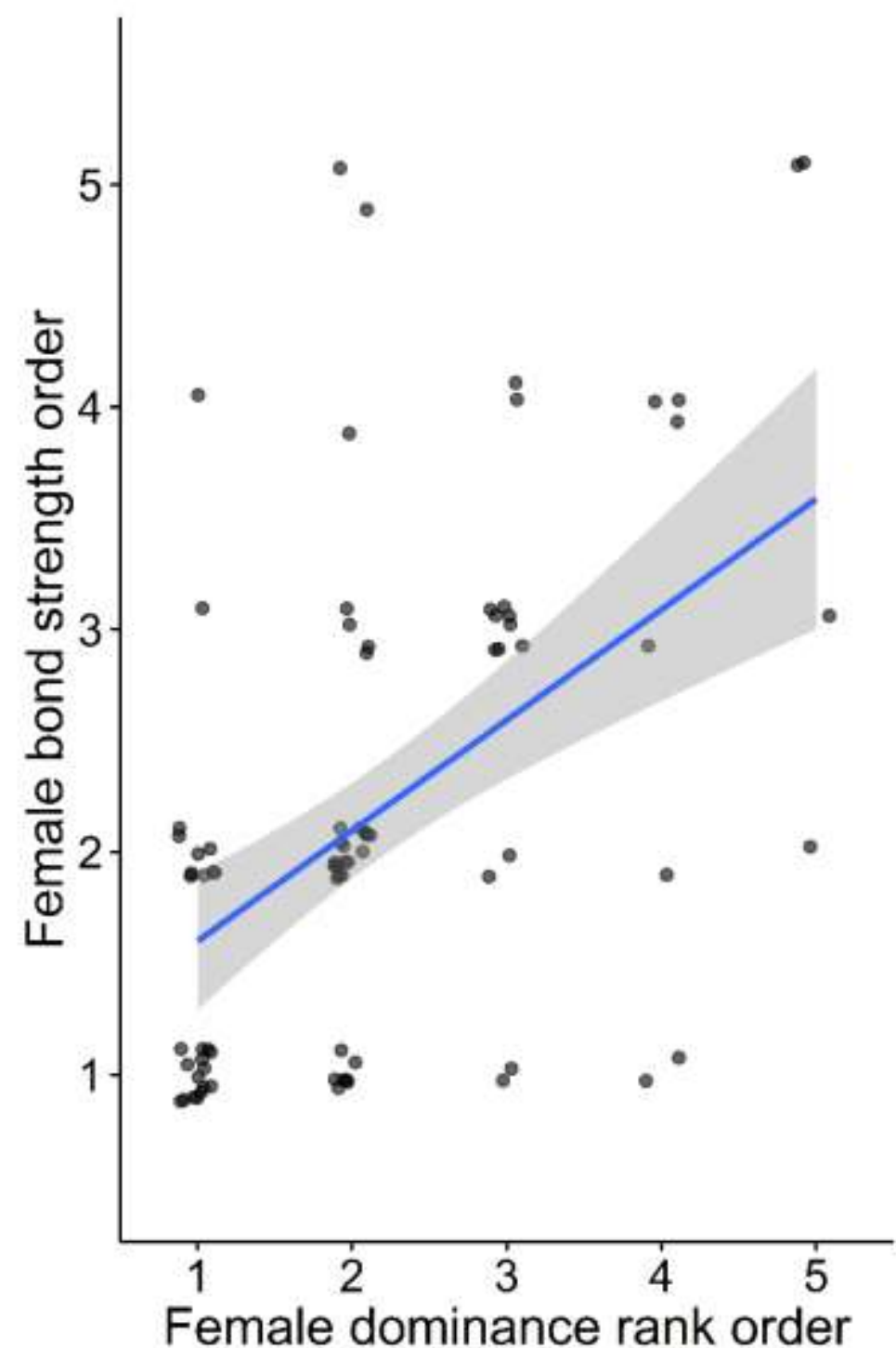

Figure 3.2: Female affiliative relationship to a male (CSI; from 1 strongest bond) predicted by female relative dominance rank within a competitive situation. The blue line indicates the predicted linear regression.

\section{Glucocorticoid metabolite levels}

We tested females in 18 competitive situations, adding up to 52 data points. Within a competitive situation, females had higher glucocorticoid metabolite levels the stronger the relationship with the top-partner compared to their competitors ( $\beta \pm \mathrm{SE}: 0.45 \pm 0.14, t=3.27$, $p<0.005$; Figure 3.3). None of the other predictors or control factors had a significant effect on glucocorticoid metabolite level of females (female grooming effort: $\beta \pm \mathrm{SE}: 0.15 \pm 0.16, t=0.94$, $p=0.35$; time in proximity: $\beta \pm \mathrm{SE}: 0.07 \pm 0.18, t=0.34, p=0.71$; reproductive state: $\beta \pm \mathrm{SE}:$ $0.05 \pm 0.27, t=-0.19, p=0.85$; season: $\beta \pm \mathrm{SE}:-0.09 \pm 0.23, t=-0.38, p=0.70$ ). 


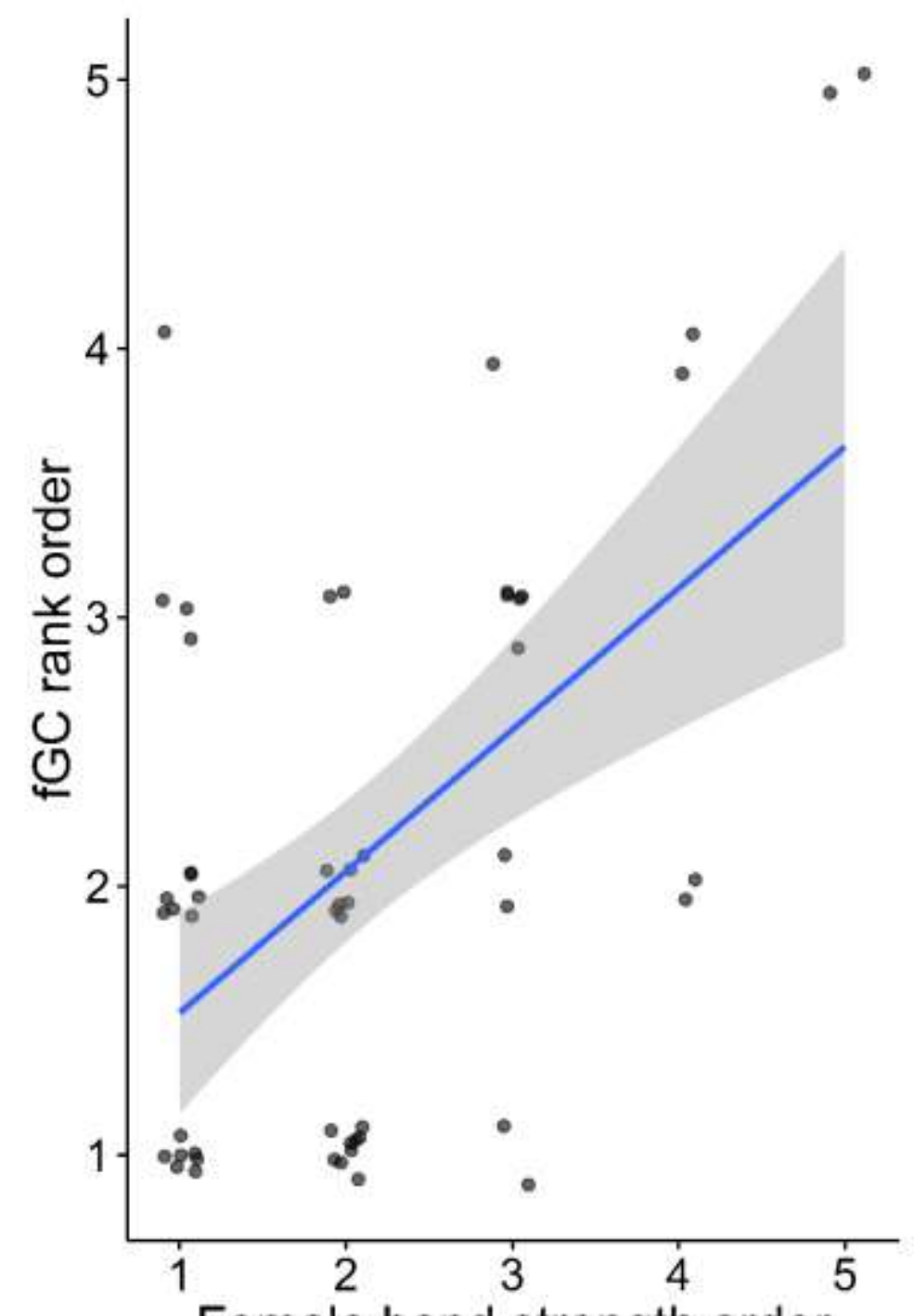

Female bond strength order

Figure 3.3: The effect of the strength of affiliative relationship (CSI; from 1 strongest bond) within a competitive situation on the female's fGC level. The blue line indicates the predicted linear regression.

\section{Male attractiveness}

Male CSI-rank, after controlling for number of males in the group, ranged between 0.02 and 0.80 (mean \pm SD: $0.49 \pm 0.19 ; N=65$ ) with high values indicating that a male was a top partner for many females. In 24 cases males were not a top partner for any female, with male rank ranging from the highest to lowest ranking male (standardized male rank, mean \pm SD: $0.51 \pm$ 0.33 ). The standardized time males spent affiliating with immatures ranged between 0 and 2.69 , with $42 \%$ of males affiliating with immatures for longer than average (mean \pm SD: $0.98 \pm$ $0.55)$.

Our model of male traits predicting a male's CSI-rank explained $59 \%$ of the variance in the response $\left(R^{2}=0.59\right.$; Figure 3.4). The higher a male's dominance rank $(\beta \pm S E: 0.43 \pm$ $0.13, t=3.37, p=0.001)$ and the more time he spent affiliating with immatures ( $\beta \pm S E: 0.26 \pm$ $0.11, t=2.34, p=0.028$ ), the higher the male's CSI-rank with females. These effects on male 
'attractiveness' were independent of each other. Season had no effect on the CSI-rank $(\beta \pm$ $S E: 0.25 \pm 0.16, t=1.50, p=0.141)$.

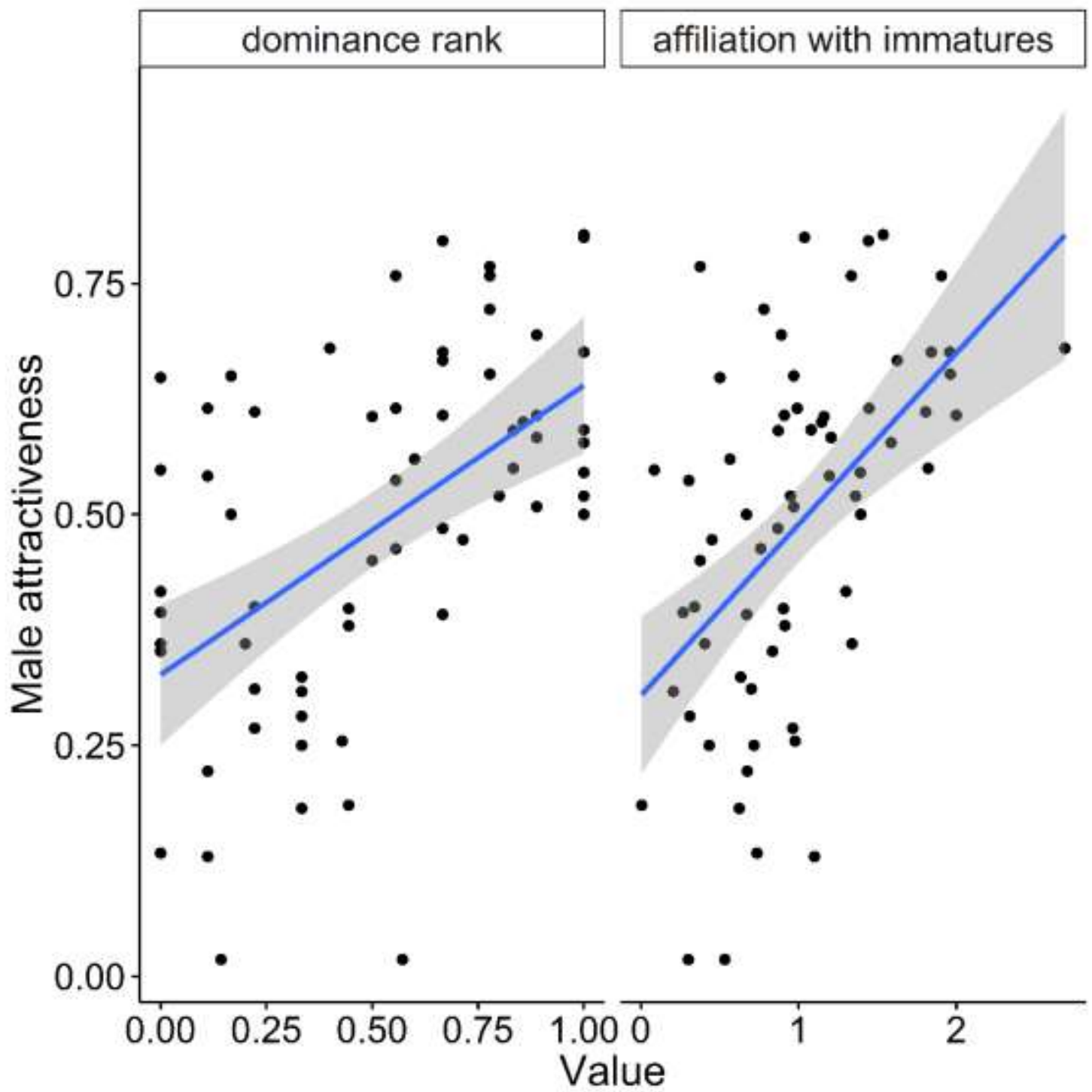

Figure 3.4: The effect of male dominance rank and time spent affiliating with immatures on a male's CSI-rank across all adult females. Original data without transformation is shown for better reading, though in the model all values were z-transformed. The lines represent the model-predicted standardized estimates with the shaded area showing the respective standard error.

\section{DISCUSSION}

In the absence of behavioral data on early hominins, non-human primates, as our closest relatives, serve to elucidate social evolution in humans (Strassmann, 1981; Chapais, 2008; Swedell and Plummer, 2012; Coxworth et al., 2015). We investigated whether the typically 
human trait of female competition over male partners may also be found in a non-human primate and show that female competition not only for mating but also for bonding partners has deep roots in human evolution.

Our results indicate a complex pattern of female competition for male partners in Assamese macaques. If competition is costly individuals might differ in their ability to deal with the costs and poor competitors might be better off by avoiding high quality partners and instead targeting low quality partners to minimize resulting costs (Fawcett and Johnstone, 2003). Such condition-dependent preferences will emerge in both sexes as result of competition for highquality partners and, if combined, can result in even more strongly assortative partner choice (Fawcett and Johnstone, 2003). The opportunity to form and maintain a bond with a male may be the resource females compete for (Palombit et al., 2001) that is limited by male social time and tolerance towards females. In our study, whenever two or more females competed over the same male their success was dictated by their dominance rank. Such rank effects are generally interpreted as indicators of strong contest competition over access to a resource (Watts, 2010).

It is in the interest of females to monopolize a male that would provide valuable resources and reliably supports her and her offspring (Buss and Schmitt, 1993; Campbell, 2004). In chacma baboons, for example, females gain protection for their offspring against infanticidal males (Palombit et al., 1997; Moscovice et al., 2009), and juveniles receive increased tolerance by biological fathers (Huchard et al., 2013). The benefits offered by associating with low-ranking males (e.g. frequent affiliative interactions and male support of offspring during agonistic interactions; Seyfarth, 1978a, 1978b; Tutin, 1979) may, for some females, be more valuable than the benefits offered by high-ranking males (e.g. support during agonistic interactions) if males do not offer both traits (Robinson, 1982). Female baboons compete for the male that most likely sired their offspring to ensure paternal investment (Palombit et al., 2001) and bonds are terminated after the highest threat of infanticide is over (Palombit et al., 1997). Female Assamese macaques benefit from social bonds with a particular male by increased support against other adult males, increased food intake rates in the male's presence (Haunhorst et al., 2017) and later male-offspring affiliation (Ostner et al., 2013) and agonistic support for the offspring (Minge et al., 2016). Accordingly, females compete for both males of high dominance rank and paternal investment, as shown in the present study. This result indicates that paternal care may not just be a consequence of malefemale association, but rather that females choose according to these traits. Though dominant Assamese macaque males have priority of access to mating partners (Schülke et al., 2010; Ostner et al., 2011, 2013; Sukmak et al., 2014), the effect of dominance is rather weak in the mating context, with a paternity skew of $30 \%$. Overall, the skew may be even lower when lifetime reproductive success is concerned. The actual behavior of a male may then be more important to females than his genetic qualities. Dominance in Assamese macaque males gives 
them power in the present to successfully protect females (Haunhorst et al., 2017), but for females the need for paternal potential seems to be rather pronounced. In humans, paternity skew is also low, because females are difficult to monopolize, leading to permanent malefemale bonds, paternal caretaking, provisioning of offspring and female partners, a reduced male-male competition for status and enhanced male tolerance (Alexander and Noonan, 1979; Strassmann, 1981).

Contrary to our prediction, females with stronger bonds to the top partner in a competitive situation did not exhibit decreased glucocorticoid metabolite levels; instead we found the opposite pattern of elevated glucocorticoid levels in these females. The results are surprising, because strong social bonds, if associated with agonistic support as in male and female Assamese macaques (Haunhorst et al., 2017) can buffer partners against the stress associated with aggressive conflicts (Hennessy et al., 2009; Young et al., 2014a). When focusing in on the comparison between those females that share the same male as their main affiliation partner we did not find the negative main effect predicted from the social buffering literature (Wittig et al., 2016). In a previous study on the same groups we have shown that decreasing levels of glucocorticoid in females are associated with increasing scores in a composite measure of overall opposite sex affiliation (Fürtbauer et al., 2014). Whether the unexpected results in this study are driven by an opposing effect of females that invest more in bonding with males have less time and energy to invest in bonding with females and thus suffer reduced social buffering effects from female affiliation or are reflective of hidden physiological costs of competition (in terms of energetic or social stressors) that we did not pick up in our study will have to be answered in future studies.

\section{Conclusions}

We found similarities between Assamese macaques and humans regarding female competition for male partners. Both species compete for males that are able to provide valuable resources potentially enhancing female reproductive success. Paternal care, a hallmark feature of human evolutionary success, seems to play a large role in female Assamese macaque partner choice. Our study provides further support for the hypothesis that pair-living in humans results from co-evolution of male and female reproductive strategies enforced mainly by female choice. More studies on non-human primates are needed to test whether this might be a general pattern, or specific to only some species under particular ecological and social conditions. 


\section{ACKNOWLEDGEMENTS}

We are grateful to the National Research Council of Thailand (NRCT) and the Department of National Parks, Wildlife and Plant Conservation (DNP) for the permission to conduct this study and their support (permit 0004.3/3618, 0002.3/2647, 0002/17, 0002/2424). We thank Kanjana Nitaya, Kitti Kreetiyutanont, Mongkul Kumsuk, Jarupol Prabnasuk and Thanee Wongnak for their help at Phu Khieo Wildlife Sanctuary, and A. Koenig and C. Borries, who developed the field site at Huai Mai Sot Yai. Special thanks goes to D. Bootros, N. Bualeng, A. Chunchaen, A. Ebenau, M. Heesen, R. Intalo, S. Jomlotwong, N. Juntuch, J. Kalbitz, M. Karlstetter, T. Kilawit, S. Macdonald, W. Nueorngshiyos, D. Pesek, N. Ponganan, S. Rogahn, P. Saaisawasthikul, K. Srithorn, M. Swagemakers, J. Wanart, B. Whitman, and T. Wisate for their excellent help in the field. Thanks to Andreas Berghänel, and Josefine Kalbitz for their assistance in data processing, and to Anja Ebenau for valuable comments on an earlier draft of the manuscript. We also thank Andrea Heistermann for conducting part of the hormone analysis and her general support in the laboratory. Our research was supported by the MaxPlanck Society, and the German Initiative of Excellence through funds to University of Göttingen and the Studienstiftung des Deutschen Volkes to $\mathrm{CBH}$. 


\section{General Discussion}

In the previous chapters, I explored opposite-sex social bonds in Assamese macaques to assess the adaptive significance of male-female relationships in a non-human primate species. I described the detailed patterns, resulting benefits for females and the occurrence of costly competition for access to male bonding partners. In the following, I will discuss the results of my research and combine them with previous research on the same population to draw general conclusions. Next, I will discuss opposite-sex relationships in a broader context, comparing several primate species regarding the differentiation and benefits found so far. I will draw a theoretical framework of how permanent pair-bonds in Assamese macaques may evolve and further discuss the implications for the evolution of the human pair-bond. Finally, I will give an overall conclusion of my study and suggest some directions for future research.

\section{THE ADAPTIVE SIGNIFICANCE OF OPPOSITE-SEX SOCIAL BONDS FOR ASSAMESE MACAQUES}

Social bonds are defined as differentiated, stable and equitable affiliative relationships (Ostner and Schülke, 2014). If affiliative relationships to specific group members have any significance for an individual, they should differ from relationships to other individuals in the group (Silk et al., 2006b) as it is well known for same-sex affiliative relationships in males and females, respectively (Cords, 2002; Silk et al., 2006b; Mitani, 2009; Kalbitz et al., 2016). Social connectedness (i.e. being connected to a large variety of individuals, irrespective of the strength of the affiliative relationship to one specific individual) may have its own advantages, but is set apart from the benefits received by specific individuals (Sapolsky and Ray, 1989; Archie et al., 2014). The strongest affiliative relationships of an individual are usually associated with benefits like coalitionary support (Silk, 1994; Silk et al., 2004; Connor, 2007; Romero and Aureli, 2008b; Schülke et al., 2010; Berghänel et al., 2011; Fraser and Bugnyar, 2012), increased tolerance (Lehmann and Boesch, 2009), enhanced offspring survival (Silk et al., 2003; Cameron et al., 2009), or enhanced longevity (Friedman et al., 1995; Silk et al., 2010b). Assamese macaques form very differentiated affiliative relationships with the same (Macdonald, 2014; Kalbitz et al., 2016), as well as with the opposite sex (Chapter 1; Haunhorst 
et al. 2016). The dyadic composite sociality index (CSI) allows for comparison of group-wide patterns as well as for defining top partners (highest CSI of one individual compared to all other members of the opposite sex in the group) for each individual. I found that the strongest affiliative relationships of an individual to the opposite sex were not always mutual, but rather that sometimes several females featured their strongest affiliative relationship to the same male. Not all individuals were the strongest bonded partner of any other group member, even when adult sex ratio was even. Both, males and females could be a top partner of several individuals of the opposite sex. That only a few individuals were top partner of many, and at the same time some individuals were top partner of none, leads to the assumption that either individuals differ in their attractiveness to others, or some individuals have the ability to gain access to more conspecifics than others do. For instance, when one male is top partner of several females, it may be that he is preferred by those females, or that he has the ability to access more females than other males do. Given that the CSI does not include any directed behavior, we cannot draw any further conclusions about initiation of relationships just from the strength of dyadic affiliative relationships. The differentiation of affiliative opposite-sex relationships was intermediate between same-sex affiliative relationships in males and females, respectively (Macdonald, 2014; Haunhorst et al., 2016; Kalbitz et al., 2016). This fact can lead to the assumption that the significance of opposite-sex affiliative relationships is either to be defined in the significance between that of male-male and female-female affiliative relationships, or that it represents a different significance for each sex (Chapter 1; Haunhorst et al. 2016).

Long-term stability of affiliative relationships may enhance predictability of a partner's behavior (Schino and Aureli, 2009). In same-sex relationships, stability is often predicted by the strength of the affiliative relationship (Silk et al., 2006a, 2012; Mitani, 2009; Kalbitz et al., 2016). How stable (i.e. long-lasting) relationships are may also provide information about the reasons of formation. Particularly in opposite-sex relationships, it is important to know whether affiliative relationships are mainly formed during the female's fertile phase as male mate guarding behavior, when dependent offspring are involved, or whether they are completely independent of any reproductive context (Seyfarth, 1978a, 1978b). I found that in Assamese macaques both male and female top 3 relationships were more stable across reproductive seasons than weaker ones (Chapter 1; Haunhorst et al. 2016). Females' relationships to the opposite sex were still more stable than males' (Figure D1; the figure was not printed in the published version of Chapter 1). Figure D1 shows that in females almost all lines (connecting the same individual's partner stability indices, PSIs, between top 3 and lesser 3 partners) head downwards, while in males the picture is not as clear. One could assume that female biased adult sex ratio favors instability in males' relationships to the opposite sex and stability in females', as the choice for males is larger than for females. However, the result held true even when sex ratio was even, indicating that females are not as variable as males in their choices 
of top 3 partners, and that for females a stable affiliative relationship with a male may be simply more important than vice versa. Females also retained their top 3 partners across nonconsecutive years, as long as males were still resident in the group. Almost all terminated relationships were due to emigration or death of the male partner. These results are in line with a former study on the same population that found group-wide spatial association patterns between males and females being stable for at least two to three years (Ostner et al., 2013). Hence, male and female Assamese macaques form relationships with each other that are independently stable for years on both the spatial and the affiliative level.

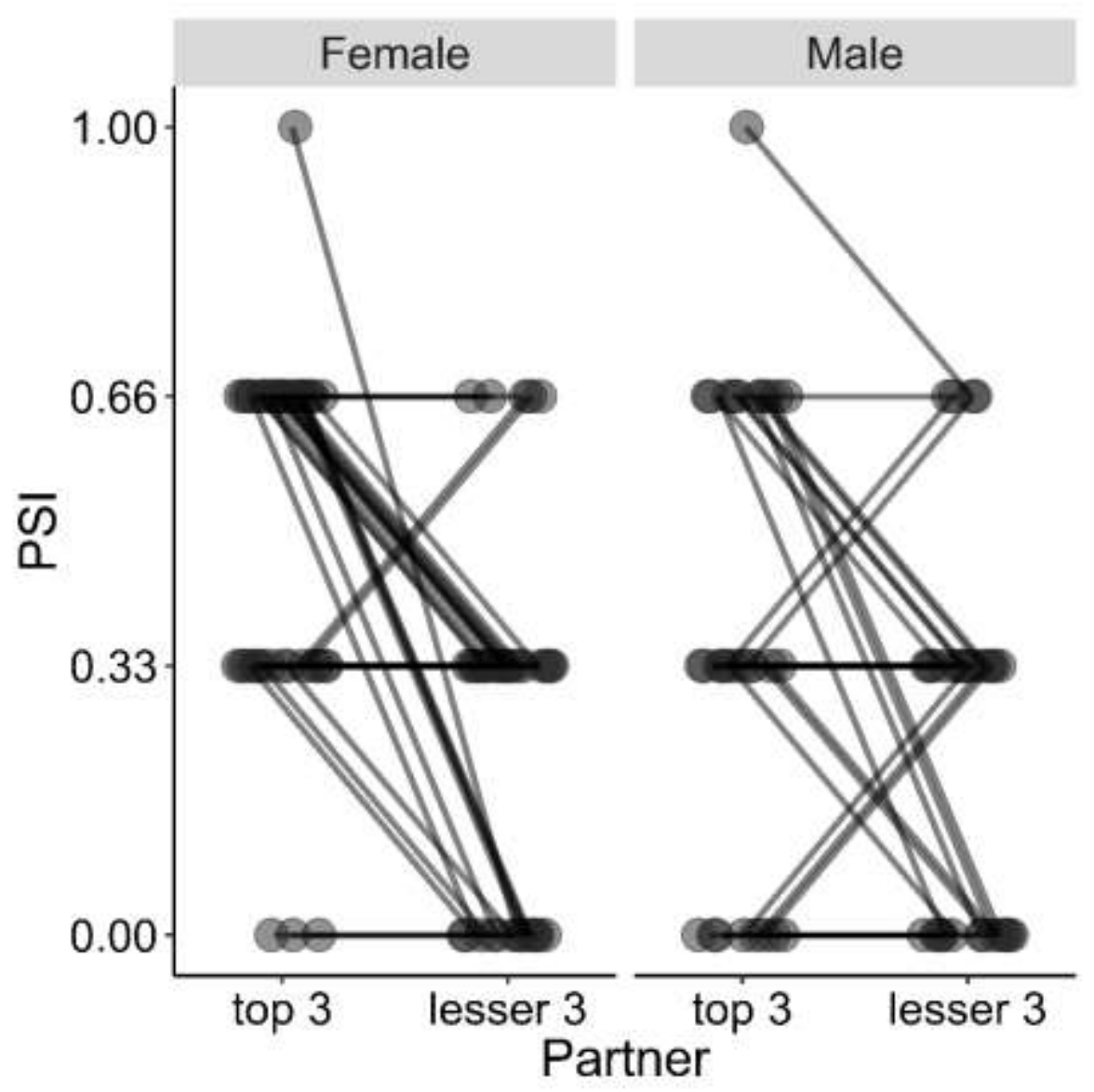

Figure D1: Stability of opposite-sex relationship categories based on the partner stability index (PSI) across consecutive reproductive seasons for males and females separately. The PSI calculates how stable a category of partners (top $3=$ three strongest relationships; lesser $3=$ three next strongest relationships following top 3 ) is in relation to time and availability of partners (Silk et al., 2012; Haunhorst et al., 2016). Each data point illustrates the PSI calculated for one individual within a respective year and group. Data points are jittered for better visualization. The lines depict the difference in stability of each individual between top 3 and lesser 3 partners. I provided the figure here, since it contains valuable information, but was not printed in the respective manuscript (Chapter 1; Haunhorst et al. 2016).

The equitability of affiliative relationships is the third indicator that should be fulfilled to call a strong affiliative relationship a social bond (Mitani, 2009; Silk et al., 2010a; Kalbitz et al., 2016). Most commonly equitability is measured by the symmetry in the direction of grooming, or approaches into close proximity (Hinde, 1983; Silk et al., 2013). In the long run, relationship equitability may not be achieved according to exchange of the same behavior, but rather as 
interchange of different behaviors that balance out at some point (Schino and Aureli, 2010b). For this reason, I will pool results of Chapter 1 (Haunhorst et al., 2016), on grooming reciprocity and symmetry, with the results in Chapter 2 (Haunhorst et al., 2017), on tenor and agonistic support, to determine the equitability of affiliative relationships in Assamese macaques.

I found that the stronger the affiliative relationship, the more likely was reciprocation of grooming (i.e. grooming received was given back at least once within the reproductive season) within a dyad (Figure D2; the figure was not printed in the published version of Chapter 1). On average, females groomed males more often and longer than vice versa, indicating a general female interest in the formation of affiliative relationships with particular males. The chances for reciprocation irrespective of the strength of affiliative relationships were higher in the mating season compared to the non-mating season. Combined with the observation that overall females groomed males longer and more often, the increased chances for reciprocation in the mating season most likely indicates male mating interests. How much time individuals invest in grooming is set by ecological and social constraints (Dunbar and Dunbar, 1988). Females may face a trade-off between social time with males and with females in the non-mating season, the time of late gestation and lactation. During lactation, females preferentially spend time with other mothers (Schülke and Ostner, unpubl. data) probably to successfully bond offspring in peer groups. Moreover, a previous study found that females with lower connectivity to males in the mating season have higher fecal glucocorticoid metabolite levels (fGC) than well connected ones (Fürtbauer et al., 2014), while in the non-mating season female-female connectedness is more important. Hence, for both sexes, grooming with opposite-sex partners in the mating season seems to have higher priority than in the non-mating season. Since association patterns in the mating season are predictive of paternity success and following male-infant bonding (Ostner et al., 2013), both sexes may put more effort in the maintenance of affiliative relationships in the mating season to either enhance paternity success, or ensure paternal care. The strength of the affiliative relationship had no linear effect on the symmetry of directed grooming, with females being responsible for most of the directed grooming time (Chapter 1; Haunhorst et al. 2016). However, more balanced than unbalanced grooming relationships featured higher CSI's, indicating that the strength of affiliative relationships may after all have some effect on the balance in grooming time, though not a strictly linear one.

Besides, behaviors may not just be exchanged in kind to balance a relationship. Other commodities, exchanged for grooming to result in an equitable relationship, are for example, agonistic support, reduced harassment, or feeding tolerance (Seyfarth and Cheney, 1984; de Waal, 1997; Barrett et al., 1999; Henzi and Barrett, 1999). For this reason, some studies include the ratio of affiliative compared to agonistic interactions and agonistic support given or received into the index used for describing relationships (Pereira and McGlynn, 1997; Ostner and Kappeler, 1999; Crockford et al., 2013, 2014). In Chapter 2 (Haunhorst et al., 2017), I used both, reduced harassment and agonistic support by males as benefits females derive from 
affiliative relationships with males. Reduced harassment by males and agonistic support against conspecifics may be beneficial for females, particularly in species with pronounced sexual dimorphism and male dominance over females (Smuts, 1983; MacCormick et al., 2012) like in Assamese macaques (Hemelrijk et al., 2008). If individuals differ widely in their resource holding potential, tolerance and support cannot be exchanged equable. I do not deny, though, that reduced harassment and agonistic support both are an important descriptive feature for relationship assessment (Silk et al., 2013). Here, I will integrate reduced harassment and agonistic support to assess relationship equitability. I found that the stronger the affiliative relationship of a dyad, the friendlier was the less harassment a female received by a male. Additionally, females received more agonistic support against conspecifics by males based on the strength of their relationship. Hence, even though females contributed more to the relationship in terms of directed grooming, the relationship can still be considered as balanced because males contributed in other ways.

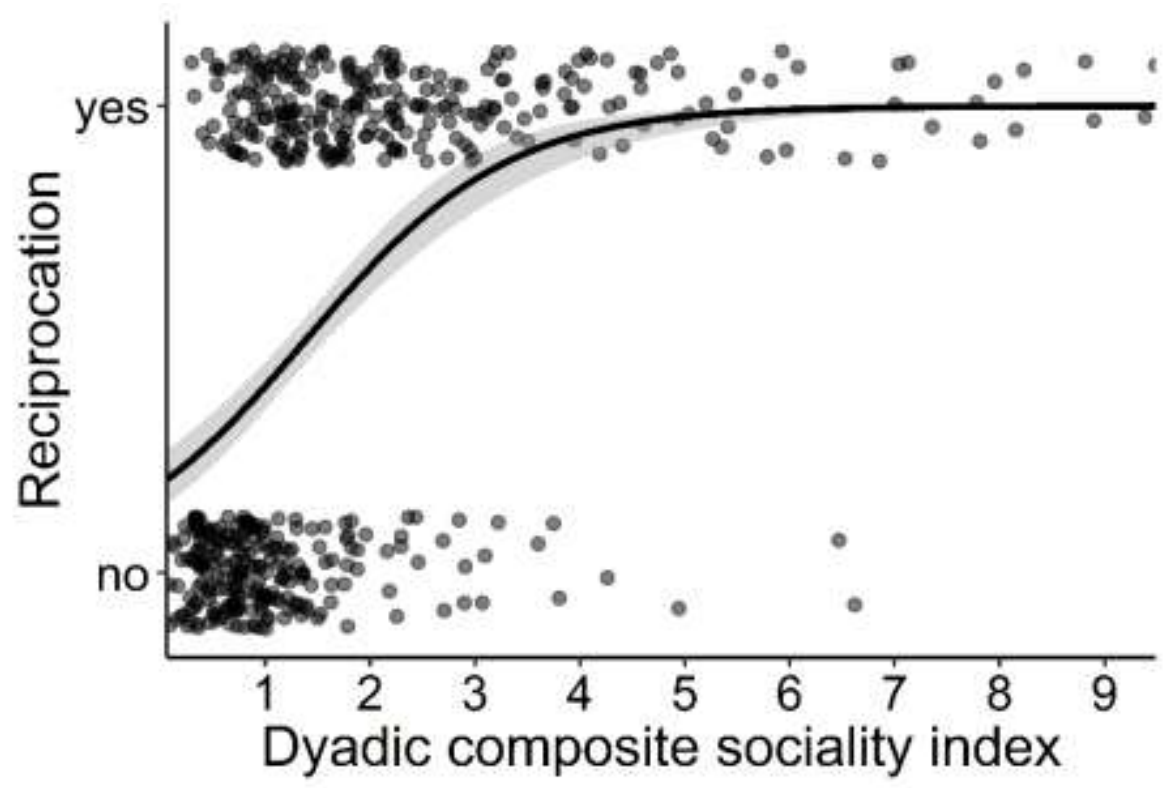

Figure D2: Probability of grooming reciprocation predicted by the dyadic composite sociality index (CSI). Each data point illustrates one male-female dyad within a respective reproductive season and group. Data points are jittered for better visualization. The line shows the resulting prediction of the applied binomial GLMM with the shaded area showing the standard error. I provided the figure here, as it contains valuable information, but was not published in the respective manuscript (Chapter 1; Haunhorst et al. 2016).

I conclude that Assamese macaques form differentiated male-female affiliative relationships, that are stable irrespective of female fertility phases and over several years, and additionally equitable in the interchange of behaviors, why, in the following, I will refer to them as social bonds (Ostner and Schülke, 2014). This conclusion leads us to the benefits both sexes may derive from forming social bonds with the opposite sex.

To increase lifetime reproductive success, individuals may increase reproductive output through shortened interbirth intervals and enhanced longevity, or increase the chances 
of offspring survival (Kuester and Paul, 1984; Fedigan et al., 1986; Bercovitch and Berard, 1993; Setchell et al., 2002; Altmann and Alberts, 2003). Female mammals face a high energy and protein demand during gestation and lactation, which is why energy intake has a strong influence on female reproductive output and maternal investment into offspring (Sadleir, 1969; Bongaarts, 1980; Strum and Western, 1982; Watanabe et al., 1992). Assamese macaque females time late gestation and following lactation to highest seasonal food availability and conception rates increase with comparatively higher food availability, demonstrating the high dependence of this species on energy intake (Heesen et al., 2013). Additionally, aggression towards the female and her offspring may corrupt physical condition and the chances for survival (Silk et al., 1981; Abbott, 1984; Wasser and Starling, 1988).

The strength of the social bond between male and female Assamese macaques predicted the friendliness of the relationship. Since all dyadic aggressive interactions were directed from the male to the female, females benefit from forming a social bond with males by gaining reduced harassment from particular males (Chapter 2; Haunhorst et al. 2017). Females also received support from males against conspecifics based on the social bond strength, particularly against males. Here, male dominance plays a large role, as $75 \%$ of agonistic support was performed by the top 3 dominant males. One may argue that males only defend mating opportunities with a particular female they spend the most time with. Contrary to that argument, the most agonistic support was observed during the non-mating season, and the time each dyad spent in a $5 \mathrm{~m}$ distance predicted the chances for agonistic support independently. The effect of opportunity may be relevant, however, the effect of the social bond on the males' behavior is statistically independent. The derived assumption is that females actually benefit from agonistic support not only in the context of mating, but also rather in a more general way. This conclusion is also mirrored in the context of feeding. The chance to find females co-feeding with particular males was predicted by the strength of their social bond, not the time the dyad spent in a $5 \mathrm{~m}$ distance. Reduced harassment can also be interpreted as increased tolerance, combining the two effects with each other. Additionally, the stronger the bond with a male the higher were the female's ingestion rates in the male's presence. That females featured increased ingestion rates when feeding with particular males could be the result of male tolerance, reduced harassment and agonistic support combined. While feeding with particular strongly bonded males, the female may not have to fear his agonism, and may even count on his protection against other males. Consequently, a female would not be interrupted in feeding as often, which is the main constraint on ingestion rates in olive baboons (Barton and Whiten, 1993). The results indicate that social bonds with males reduce feeding competition for female Assamese macaques. An additional benefit is agonistic support against conspecific males, particularly when dependent offspring is involved. Interestingly, same-sex social bonds lower feeding competition in females (Heesen et al., 2014) and increase coalitionary support in male Assamese macaques (Schülke et al., 2010). Hence, opposite-sex 
bonds have similar benefits for females as same-sex bonds for females and males, respectively, which would also explain opposite-sex relationships being intermediate between both same-sex differentiations.

Male-female association patterns in Assamese macaques are predictive of the paternal success of a male with the respective female, and the male's future association with his biological offspring (Ostner et al., 2013). Males may thus benefit in terms of reproductive output and the chances to provide 'true paternal care', enhancing his biological offspring's chances for survival. In line with this argument, males in this species support strongly bonded immatures in agonistic interactions, not only in times of highest risk of infanticide (Minge et al., 2016). While males may increase reproductive success through social bonds with females by securing mating opportunities and paternity, females may increase reproductive success through lowered feeding competition, and both sexes by enhanced paternal care. Females may thus benefit twofold from forming social bonds with males. Whether social bonds actually translate into increased reproductive success for males and females remains to be clarified.

The benefits females receive through social bonds with males (i.e. reduced feeding competition, agonistic support, paternal care), combined with the finding that a few males were the top partner of several females, evoke the question, whether particular male partners may be a more valuable resource than others. If so, females are supposed to compete for male partners as manifestation of resource competition (Stockley and Bro-Jørgensen, 2011). Male attractiveness for females seems to vary in Assamese macaques, as several females have the same male as a top partner, while other males are not top partner of any female (Chapter 1; Haunhorst et al. 2016). In the case that several females shared the same male as top partner, I did define a competitive situation. Female dominance rank had a strong influence on access to male partners, at least on an individual if not on a group-wide pattern (Chapter 3), whereas male top partners varied strongly in dominance rank. The results indicate competition for access to particular males. A previous study showed that failing to connect with males in the mating season is stressful for females (Fürtbauer et al., 2014), leading to the conclusion that females are in need of a social bond. So far it was unknown, whether bonding to males in general or to specific partners would have an effect on the female's stress response. Now, we can conclude that though a lacking connectedness in males creates higher glucocorticoid metabolite levels in females (Fürtbauer et al., 2014), females also suffer from increased glucocorticoid metabolite levels when forming the strongest bond with a male compared to other females in a competitive situation (Chapter 3 ).

For females, competition for males seems to be costly even when winning in a competitive situation. For this reason, they should choose male partners that can provide the needed benefits. Male dominance rank had a strong influence on the disposition to provide agonistic support to females (Chapter 2; Haunhorst et al. 2017). Dominant males can not only support females more effe 
ctively but may also have better access to resources (Watts, 2010). On the other hand, paternal care may enhance offspring survival and males may differ in their paternal care-giving disposition. In Assamese macaques, both current dominance rank and former affiliation with immatures had an independent positive effect on the male's 'attractiveness' for females (measured as average CSI rank across all females; see details in Chapter 3). Considering males' dispositions to provide agonistic support that is strongly favored by male dominance rank (Chapter 2; Haunhorst et al. 2017) on the one hand, and paternal care on the other hand (Ostner et al., 2013; Minge et al., 2016), females may face a trade-off regarding their choice for bonding partner and their individual needs. That individual females actually differ in their needs, explains the variation of male dominance rank in those males that females compete for.

Overall, the study suggests that social bonds between the sexes are very similar to same-sex relationships. The formation of female bonds lowers feeding competition within the group (Heesen et al., 2014). Opposite-sex social bonds in Assamese macaques seem to further lower feeding competition for females. Social bonds with same-sex partners increase the likeliness to successful coalition formation in males (Schülke et al., 2010). Female Assamese macaques derive the same benefits by forming social bonds with particular males. My study suggests that the significance of opposite-sex social bonds for female Assamese macaques may be similar to the significance of same-sex social bonds.

\section{SPECIES COMPARISON}

I reviewed literature on wild or free ranging old world anthropoid populations, where sufficient data was available to ensure reliable species comparison. There is only a scarce number of studies published that reported to not have found special relationships between males and females (e.g. Small 1990a), which is why the real number of species featuring differentiated opposite-sex relationships in multimale-multifemale groups may be much higher. Building on studies that provide enough data to serve for a more or less reliable species comparison (see General Introduction and Table D1), I focused on those traits that have the most influence on or are the best indicator for the adaptive significance of opposite-sex social relationships for females (as in detail introduced above). The differentiation of affiliative relationships indicates individual preferences, and the stability shows how dependent affiliative relationships are on female reproductive state. The direct benefits females may receive from bonded males in terms of feeding tolerance, passive protection (by the male's presence without the male actively 
intervening) or active support (i.e. active aggressive intervention in ongoing conflicts) indicate the importance of the bond for females at a present state. Male care provided, by affiliation with female offspring, higher feeding tolerance, passive protection or active support, benefit the female not only immediately but also by enhancing offspring survival and development. Immediate benefits and male care, as well as the risk of infanticide, should have an influence on the effort females put into a relationship by active maintenance and competition with other females. Male care, on the other hand, should be affected by the risk of infanticide and paternity certainty, which in turn is influenced by male monopolization potential due to reliable reproductive signals of females and the seasonality of breeding.

Table D1: Comparison of species that live in large multimale-multifemale groups with promiscuous mating system, regarding reproductive traits (Repr), differentiation and stability of male-female affiliative relationships (Bond), female investment (Inves), benefits for females (Benefit), and male care provided to biological or non-related offspring (Male care). The trait might either be evident $(\mathrm{Y})$, non-existent or very rare $(\mathrm{N})$, probably occurring (?) or not reported (blank).

\begin{tabular}{|c|c|c|c|c|c|c|c|c|c|c|c|c|c|c|}
\hline \multirow[t]{2}{*}{ Species } & \multicolumn{2}{|c|}{ Bond } & \multicolumn{3}{|c|}{ Benefit } & \multicolumn{4}{|c|}{ Male care } & \multirow[b]{2}{*}{ 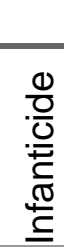 } & \multicolumn{2}{|c|}{ Inves } & \multicolumn{2}{|c|}{ Repr } \\
\hline & 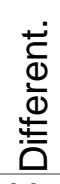 & $\begin{array}{l}\frac{0}{0} \\
\frac{\mathbb{T}}{0} \\
\text { D) }\end{array}$ & 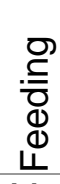 & $\begin{array}{l}\overline{0} \\
\mathbb{d} \\
\text { o } \\
\text { D. }\end{array}$ & 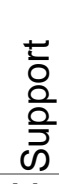 & 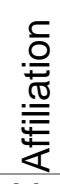 & 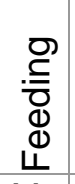 & $\begin{array}{l}\bar{d} \\
\bar{d} \\
\overline{0} \\
\overline{0}\end{array}$ & $\begin{array}{l}\frac{5}{0} \\
\text { 응 } \\
\text { ஸ }\end{array}$ & & 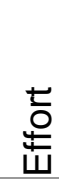 & $\begin{array}{l}\stackrel{0}{ \pm} \\
\stackrel{0}{E} \\
\stackrel{0}{0}\end{array}$ & $\begin{array}{l}\overline{\widetilde{v}} \\
\overline{\frac{D}{n}} \\
\bar{c}\end{array}$ & 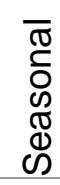 \\
\hline Homo sapiens ${ }^{1}$ & $\bar{Y}$ & $\mathrm{Y}$ & $\bar{Y}$ & $\overline{\mathrm{Y}}$ & $\mathrm{Y}$ & $\mathrm{Y}$ & $\bar{Y}$ & $\bar{Y}$ & $\mathrm{Y}$ & $\bar{Y}$ & $\bar{Y}$ & $\mathrm{Y}$ & $\mathrm{N}$ & $\mathrm{N}$ \\
\hline Pan troglodytes ${ }^{2}$ & $Y$ & $Y$ & $Y$ & $\mathrm{Y}$ & Y & $\mathrm{N}$ & $\mathrm{N}$ & $\mathrm{N}$ & $\mathrm{N}$ & $\mathrm{Y}$ & $\mathrm{Y}$ & $\mathrm{N}$ & Y & $\mathrm{N}$ \\
\hline Papio anubis ${ }^{3}$ & $\mathrm{Y}$ & $\mathrm{Y}$ & $\mathrm{N}$ & $\mathrm{Y}$ & $\mathrm{N}$ & $\mathrm{Y}$ & $\mathrm{N}$ & $\mathrm{Y}$ & $\mathrm{N}$ & $\mathrm{Y}$ & $\mathrm{Y}$ & $\mathrm{Y}$ & $\mathrm{Y}$ & $\mathrm{N}$ \\
\hline Papio ursinus ${ }^{4}$ & $\mathrm{Y}$ & $\mathrm{N}$ & $\mathrm{N}$ & Y & $\mathrm{N}$ & Y & $\mathrm{Y}$ & $\mathrm{Y}$ & $\mathrm{Y}$ & $\mathrm{Y}$ & $\mathrm{Y}$ & $\mathrm{Y}$ & $\mathrm{Y}$ & $\mathrm{N}$ \\
\hline Papio cynocephalus ${ }^{5}$ & $\mathrm{Y}$ & $\mathrm{N}$ & & $\mathrm{Y}$ & $\mathrm{N}$ & $\mathrm{Y}$ & & $\mathrm{Y}$ & $\mathrm{Y}$ & $\mathrm{N}$ & $\mathrm{Y}$ & $\mathrm{Y}$ & $\mathrm{Y}$ & $\mathrm{N}$ \\
\hline Macaca nigra $^{6}$ & & & & & & $Y$ & & $?$ & $\mathrm{~N}$ & $\mathrm{Y}$ & & $\mathrm{N}$ & $\mathrm{Y}$ & $\mathrm{N}$ \\
\hline Macaca sylvanus ${ }^{7}$ & $\mathrm{~N}$ & $\mathrm{~N}$ & $\mathrm{~N}$ & $\mathrm{~N}$ & $\mathrm{~N}$ & $\mathrm{Y}$ & $\mathrm{N}$ & $\mathrm{N}$ & $\mathrm{N}$ & $\mathrm{N}$ & $\mathrm{N}$ & $\mathrm{N}$ & $\mathrm{Y}$ & $\mathrm{Y}$ \\
\hline Macaca mulatta ${ }^{8}$ & Y & $Y$ & $\mathrm{Y}$ & $\mathrm{N}$ & $\mathrm{Y}$ & Y & $\mathrm{N}$ & $?$ & $\mathrm{~N}$ & $\mathrm{~N}$ & $Y$ & $\mathrm{Y}$ & Y & Y \\
\hline Macaca fuscata9 & $Y$ & $\mathrm{Y}$ & $\mathrm{Y}$ & $\mathrm{Y}$ & $Y$ & $\mathrm{Y}$ & & $?$ & $\mathrm{~N}$ & $\mathrm{~N}$ & $Y$ & $\mathrm{Y}$ & $Y$ & $\mathrm{Y}$ \\
\hline Macaca sinica ${ }^{10}$ & $\mathrm{Y}$ & & & & & $\mathrm{Y}$ & & & & $\mathrm{N}$ & $\mathrm{Y}$ & $\mathrm{Y}$ & $\mathrm{N}$ & $\mathrm{Y}$ \\
\hline Macaca assamensis ${ }^{11}$ & $\mathrm{Y}$ & $\mathrm{Y}$ & $\mathrm{Y}$ & Y & $\mathrm{Y}$ & Y & & $\mathrm{Y}$ & $\mathrm{Y}$ & $\mathrm{N}$ & $\mathrm{Y}$ & $\mathrm{Y}$ & $\mathrm{N}$ & $\mathrm{Y}$ \\
\hline
\end{tabular}

1: Daly and Wilson, 1994; Friedman et al., 1995; Kaplan et al., 2000; Fawcett and Johnstone, 2003; Brewis and Meyer, 2004; Campbell, 2004; Marlowe, 2004; Schmitt, 2005; Brown et al., 2009; Fernández-Duque et al., 2009; Stockley and Campbell, 2013; Chapais, 2013

2: Arcadi and Wrangham, 1999; Deschner et al., 2004; Lehmann et al., 2006; Kahlenberg et al., 2008; Langergraber et al., 2013; Machanda et al., 2013

3: Smuts, 1985; Lemasson et al., 2008; Higham et al., 2009

4: Palombit et al., 1997, 2001; Weingrill et al., 2003; Moscovice et al., 2009, 2010; Huchard et al., 2013; Baniel et al., 2016

5: Buchan et al., 2003; Gesquiere et al., 2007; Nguyen et al., 2009; Archie et al., 2014

6: Reed et al., 1997; Higham et al., 2012; Kerhoas et al., 2014, 2016; Marty et al., 2015

7: Kuester and Paul, 1986; Small, 1990a, 1990b; Paul et al., 1996; Ménard et al., 2001; Heistermann et al., 2005; Brauch et al., 2007

8: Chapais, 1983a, 1983b; Hill, 1990; Manson, 1994; Dubuc et al., 2009; Kulik et al., 2011; Langos et al., 2013

9: Takahata, 1982; Tsukahara, 1990; Nunn, 1999; Majolo et al., 2010

10: Dittus and Ratnayeke, 1989; Ratnayeke, 1994; Nunn, 1999

11: Fürtbauer et al., 2011a; Ostner et al., 2013; Haunhorst et al., 2016, 2017.; Minge et al., 2016

Comparing the reviewed species, Table D1 shows that most of them seem to exhibit the formation of differentiated affiliative relationships between males and females. This universal pattern across most species is a first indicator that opposite-sex affiliative 
relationships are significant to at least one of the sexes. Stability on the other hand, varies across species. Chacma and yellow baboons do not form very stable relationships, instead affiliation is terminated when the female's offspring ages past the highest risk of infanticide or dies (Palombit et al., 1997; Nguyen et al., 2009). In all other species, though, affiliative relationships are stable across females' reproductive state. This indicates, that the affiliative relationship offers benefits to the female, and probably the male as well, that are not only connected to the presence of dependent offspring. In female olive baboons, the rate of aggression received is highly correlated with the time spent with males, but the affiliative relationship between the two reduces the aggression received by that male (Smuts, 1985). Male tolerance seems to be a rather common but by no means universal outcome of the formation of affiliative relationships between the sexes. Tolerance entails high benefits for females, in terms of reduced feeding competition and reduced harassment (Melis et al., 2006; Carne et al., 2011; Tiddi et al., 2011; Borgeaud and Bshary, 2015). For males, tolerance of females in their proximity may generally not be very costly. This arrangement may also account for the universal pattern of protection reported. Females may merely be considered to be protected in the male's presence, if females actively seek the male's proximity when potential threats from conspecifics arise (Nguyen et al., 2009), or because the male responds more strongly to auditory stimuli (Palombit et al., 1997; Lemasson et al., 2008). There are not necessarily reports of the male actively intervening in agonistic conflicts in favor of the female. As long as the male's presence alone suffices to fend off rivals without actively supporting the female, the male does have little costs but the female high benefits. Male dominance rank should then be an attractor for females, for the lower likelihood of dominant males being aggressed by conspecifics.

Because agonistic support (i.e. intervening aggressively in agonistic conflicts in favor of one of the opponents) against conspecific males is much more likely to involve costs for males (Silk, 1994), we find it far less among the here reported species. Excessive fights between males could end fatal or at least carry the risk of injury, which might in turn be a threat to male dominance. For baboons, this may be reason enough to avoid overt aggression with other males to aid females. The same should apply to crested macaques. In rhesus macaques, males cue for dominance rank and form coalitions with females that may retain male dominance rank (Chapais, 1983a), leading to a male interest to form coalitions with females in general and to form coalitions with high-ranking females in particular. Assamese macaque males attain dominance rank by forming coalitions with bonded males (Schülke et al., 2010). For this reason, male dominance rank may not be threatened directly in a single fight with another male, particularly when the bonded partner can interfere in the ongoing male dispute. Male social bonds and stable coalition partners may favor agonistic aid of males towards females in this species. As female aggression is generally considered to be less violent (Stockley and Bro-Jørgensen, 2011; Clutton-Brock and Huchard, 2013a, 2013b; Stockley and 
Campbell, 2013) it may involve lower costs for the opponent females. Males may not bother to intervene aggressively, in particular when their own intervention may trigger aggressive intervention by another male in return. In contrast, male chimpanzees are known to intervene in aggressive interactions between females. Since chimpanzees live in male-bonded groups with female dispersal, males gain mating opportunities through newly immigrating females. Females, on the other hand, suffer costs through additional females in terms of feeding competition. Accordingly, female conflicts in chimpanzees are more violent and males need to intervene aggressively in female-female conflicts to preserve their own interests (Kahlenberg et al., 2008). Male bonds in chimpanzees may then further reduce the involved risks for intervening males.

In summary, males often may not receive enough immediate benefits from females themselves to overly invest into their protection. Only in a few species, agonistic support seems to pay off for males. Sexual selection theory favors males increasing their reproductive output foremost by fertilizing as many females as possible (Trivers, 1972). In primates, slow life histories and limited access to females males may increase reproductive output by providing paternal care for their biological offspring, enhancing their survival chances, and increasing at the same time their own indirect reproductive fitness (Clutton-Brock, 1988). Hence, males may invest more into their (potential) offspring than in females.

Passive protection of immatures via tolerating their proximity and unintentionally preventing other males from committing infanticide may not be very costly for males, and thus a more universal pattern among non-human primates. In species with high rates of infanticide, like chacma baboons, males still provide costly support against other males even if paternity is not certain, because the risk of injury for unrelated immatures compared to the risk of not protecting uncertain but potential biological offspring may be the better trade-off for male fitness (Moscovice et al., 2009). Paternal care has been attributed to the existence of sexual swellings, because they are supposed to narrow down paternity certainty based on mating history (Alberts and Fitzpatrick, 2012). In line with this argument, active agonistic support for immatures was only reported in those species where males most likely can distinguish their biological offspring from other immatures (i.e. chacma baboons: Huchard et al. 2013, yellow baboons: Buchan et al. 2003). In contrast to most other non-human primates living in large multimale-multifemale groups (Nunn, 1999), Assamese macaque females successfully conceal their ovulation from males (Fürtbauer et al., 2011a). Besides concealed ovulation, males may have information about the identity of their biological offspring (Ostner et al., 2013), which is mirrored in the fact that costly agonistic support provided by males in favor of immatures is considered to be connected to high paternity certainty (Alberts and Fitzpatrick, 2012). In this species, the number of copulations in mating season is concordant with paternity success (Ostner et al., 2013), despite the fact that females successfully mate with all males of the group during mating season (Fürtbauer et al., 2011a), leaving uncertainty of paternity for 
all males in the group. The social bond may serve as mediator to ensure paternity certainty for the male, as well as successful bonding of immatures to biological fathers for the female. If the social bond enhances male paternity success and the female in turn gains paternal care for her offspring, both sexes benefit from the arrangement.

Females establish affiliative relationships with particular males and invest in directed grooming time, if only to gain male tolerance for herself or her offspring as a reward. Females can then be close to males and reap the benefits of being near a male, without suffering the consequences of increased aggression (Smuts, 1985). As females, in all species, benefit from affiliating with a particular male at least in one way, the higher females' investment by grooming with a male may be a consequence of reciprocal behavior (Schino and Aureli, 2010b).

Female competition for male partners is a surprisingly common feature, though not easily found in the reported results. Considering the formerly mentioned arguments, it may be enough for females to be close to any male, without the need for competition for particular individuals. However, males vary in their ability to provide passive protection or access to resources based on dominance rank (Watts, 2010). Males may be able to provide passive protection to more than one female, making competition unnecessary. Still, females often cue for access to males with dominant females in first line. Females may not actually compete for the resource 'male protection' but rather for the resource 'male time' (Palombit et al., 2001). Male social time is generally restricted by the time needed for feeding, and additionally by the social time needed to maintain relationships with other males or immatures. Hence, not the outcome of the affiliative relationship (e.g. passive protection) but its establishment may be the reason for competition between females, because not all females can groom the same male at the same time. Female chacma baboons compete for those males that have most likely sired their offspring (Palombit et al., 2001). It was believed that chacma females only start establishing a relationship after conception (Palombit et al., 1997). Recent evidence shows that affiliative relationships between males and females in chacma baboons already exist before conception and are stable past parturition (Baniel et al., 2016). Female competition may then also start before the most likely sire of offspring is established. Another study found, that in chacma baboons females may be more attracted to males that spend more time with immatures (Seyfarth, 1978a). Perhaps chacma baboon females already compete for access to males potentially providing paternal care, like in Assamese macaques. Detailed reports on female competition from other species are non-existent though, and so far no general conclusions on the causes, mechanisms or consequences of female competition can be drawn.

Based on the traits shown in Table D1, Assamese macaques show striking similarities with humans. Both species merely differ in seasonal reproduction featured in Assamese macaques. They may also be similar to humans in regards of competition (see Chapter 3 ). 
Female humans and Assamese macaques, face the trade-off between power (or high dominance rank) and a high degree of commitment (or potential for paternal care) as highly attractive traits in male partners (Chapter 3; Strassmann 1981). In both species, commitment may outcompete power, though the combination of these two traits is the most worth striving for (Campbell, 2004). Commitment in Assamese macaques may be interpreted as a stable and therefore reliable social bond, from which both males and females gain benefits. In humans, this kind of stable, and reliable, social bond that usually goes with commitment on both sides, but including a sexual relationship, is defined as permanent pair-bond (Quinlan and Quinlan, 2007).

\section{ASSAMESE MACAQUES AS MODEL ORGANISM FOR THE EVOLUTION OF HUMAN PAIR-BONDS}

In the following, I will combine hypotheses on the evolution of the human pair-bond and concealed ovulation to create a scenario how permanent pair-bonds may evolve or have evolved in Assamese macaques or humans, respectively. I will start with the assumption that in humans concealed ovulation evolved (or rather reliable sexual signals got lost) before the pair-bond evolved (Strassmann, 1981), like in Assamese macaques for reasons not debated here (but see Fürtbauer 2011 for an elaborated theory). I will develop a theory how pair-bonds may evolve directly from multimale-multifemale groups with promiscuous mating system. Traits already featured by Assamese macaques (as introduced previously; Figure D3) will serve as baseline, and changes in ecological conditions as catalysts for evolutionary progression.

In Assamese macaques, the current social organization, social structure, and mating system (multimale-multifemale groups, promiscuous, social bonds between all sexes) may serve both sexes best in terms of maximizing reproductive output. The existence of dry and rainy season and the resulting seasonality in food availability favors seasonal breeding in females to time birth and lactation to highest food availability (van Schaik and van Noordwijk, 1985; Heesen et al., 2013). Given the hypothetical scenario that (for example due to climate change) seasonality may be less pronounced and food availability more or less spread evenly across the entire year, females may lose their strictly seasonal ovulation patterns (Fairbanks and McGuire, 1984). Though macaques may be less flexible to shift seasonal ovulation patterns than other primate species (Silk et al., 1981), Assamese macaques already feature prolonged sexual receptivity across four months during mating season (Fürtbauer et al., 2011a). Additionally, females may conceive later, and at the same time more often, when changed conditions favor reproduction. For instance, in 2013 infants were born between February and August, indicating a prolonged mating season, and though unexpected, the 
female with latest parturition gave birth again only 10 months later (personal observation; Ostner and Schülke, unpubl. data). Aseasonal food availability may also result in less clumped food patches and females' need to disperse further for foraging, leading to lowered male herding potential (Lukas and Clutton-Brock, 2013).

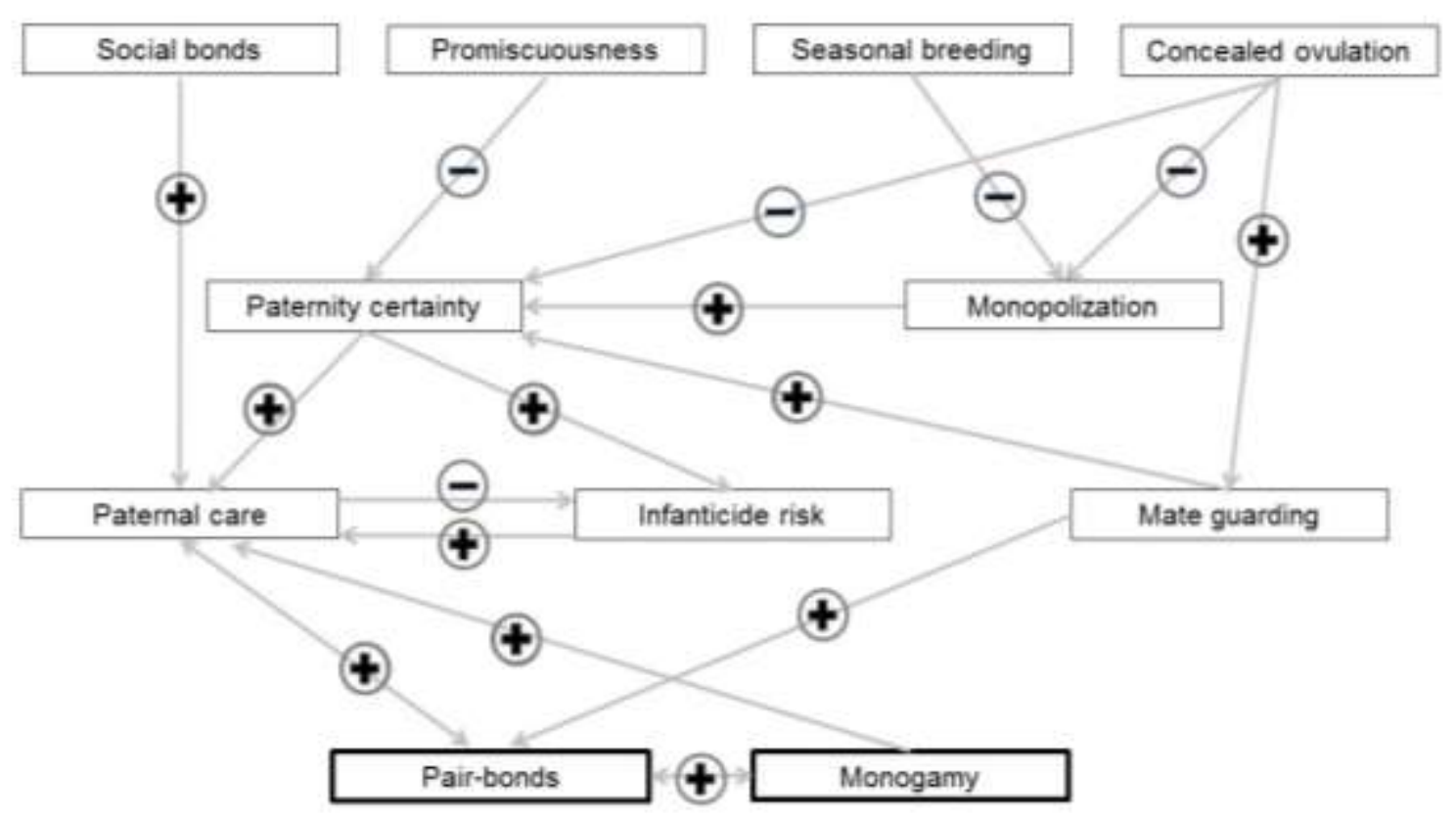

Figure D3: Model for the evolution of pair-bonds in Assamese macaques. The current status of social structure and mating system in Assamese macaques at the top line, with its positive (+) or negative (-) influence on other traits, leading to pair-bonds. Details about necessary changes to lead to permanent pair-bonds can be found in the text.

Female Assamese macaques would still feature concealed ovulation, resulting in lowered male-male competition for mating (Andelman, 1987; Marlowe and Berbesque, 2012), followed by a decreasing dominance skew in mate guarding ability of males. In turn, operational sex ratio may become more male-biased, because females spread receptive phases throughout the year leading to only a few fertile females at the same time, while the number of males would be constant. Male biased operational sex-ratio is supposed to be a prerequisite for the formation of stable pair-bonds (Quinlan and Quinlan, 2007). In human societies that are classified as monogamous, or serial monogamous, operational sex ratio is usually rather male-biased, while for polygynous populations the opposite holds true (Coxworth et al., 2015). On the other hand, a male biased operational sex-ratio leads again to enhanced male mating competition (Kvarnemo and Ahnesjö, 1996). The intensity of male competition should favor males successfully defending an existent bond, rather than searching for a new mate (Parker, 1974; Quinlan and Quinlan, 2007), particularly when concealed ovulation complicates finding mates at the time of ovulation. Males may then increase their chances for paternity by extending mate guarding for longer periods in which a female may be fertile (Palombit, 1999), leading to an enhanced certainty of paternity. In Assamese macaques, 
association patterns in the mating season already predict male paternity success with that female across all male dominance ranks (Ostner et al., 2013). At the current state dominant males still have priority of access to fertile females. If male monopolization potential is further lowered, all males may adopt strategies of prolonged associations over longer times to increase paternity success. Males should defend rather long-term mating relationships when facing costs by abandoning that relationship to search for another mate (Quinlan and Quinlan, 2007; Kokko and Jennions, 2008). Time may not be the restrictive condition, given that groupliving is still enforced, because it holds benefits in terms of lowered predation risk and intergroup competition (Isbell, 1994; Sterck et al., 1997). However, if most females in a group are already taken by another male finding a new mate may be more complicated, resulting in unavoidable fighting risk. Additionally, in almost even operational sex ratios, female choice of partners may further lower male access to mates. Hence, in this scenario male access to multiple fertile females would be very low and abandoning an existent relationship may result in the female being fertilized by another male, while the former partner may not find a new mate.

Aseasonal breeding may be followed by the females' needs to rely on increased male provisioning to overcome food scarcity in times of high energy demands (i.e. birth and lactation) (Marlowe, 2003). In Assamese macaques, males do not provision females actively, but males may invest, or at least they do provide tolerance, into the female gaining more energy. This would lead to female partner choice based on traits other than dominance rank, but rather the male's willingness to provide paternal care (Strassmann, 1981; Geary, 2000). Given that males (if only accessing one fertile female) can only increase reproductive success, if the mother is in the physical condition to produce viable offspring, males should provide increased investment for the female during gestation and lactation. During lactation, the male may contribute the most to enhance his reproductive success by providing paternal care for the offspring he most likely sired (Clutton-Brock, 1988). Paternity confusion would then be lower than in a strictly promiscuous mating system, as males may have better knowledge of the female's mating history. This would be followed by an increase in male infanticide because most males can be certain of not being the sire of most of the infants. Hence, the male's investment in offspring protection would be mandatory to fend off potentially infanticidal males. Reappearing risk of infanticide may again lead to even tighter bonding between male and female during the highest risk of infanticide (van Schaik and Dunbar, 1990). Male care for female and offspring may also result in shortened interbirth intervals in females (cf. van Schaik and Dunbar 1990). When females reach fertility faster because of male help, the male may increase his own reproductive success further by staying with the same female, finally resulting in a permanent pair-bond.

In humans now, strong male bonds may enable large societies consisting of multiple family groups (Chapais, 2013). In Assamese macaques, social bonds between adult males 
are stable over several years (Kalbitz et al., 2016) and predictive of male-male coalitions (Schülke et al., 2010). Strong male bonds may increase successful defense of territories and hence overall female reproductive output through higher access to food resources (Boesch and Boesch-Achermann, 2000; Aureli et al., 2006). Additionally, for males within-group competition is lowered (Ostner and Schülke, 2014), and successful protection against incoming infanticidal males enhanced (van Schaik, 1996).

Predictability of the outcome of future interactions with the partner is important for the formation of permanent pair-bonds (Weinrich, 1977). In non-human primates emotional bookkeeping may allow long-term reciprocation between two individuals and create an environment of trust with for both individuals predictable interactions without advanced cognitive abilities (Schino and Aureli, 2010b, 2010c). The already established opposite-sex social bond in Assamese macaques may function as mediator to permanent pair-bonding, by means of predictability of future interactions with the partner. Male care, in terms of agonistic support and feeding tolerance, for females (and offspring) already exists in this species, although permanent pair-bonds are not presently existent. Male bonding is very pronounced in Assamese macaques, which would simplify the transition to rather male-bonded societies. So far, ecological conditions may favor the established social as well as mating system in Assamese macaques, but the traits required to switch social structure and mating system towards a permanently pair-bonded one are already existent.

Humans are the only species that build societies of multiple males and females living together, but with reproductive units that are entirely or primarily monogamous (Chapais, 2013). Considering that humans may also be the most successful extant species on earth, it is surprising that not more species adopted this system - in case that there is a connection. The permanent pair-bond, generated or followed by paternal care and male provisioning, builds the baseline for the development of large brains with increased cognitive abilities (Kaplan et al., 2000). Concealed ovulation in turn may have played a major role in the evolution of pair-bonds (Strassmann, 1981). The loss of this very distinctive and costly (Nunn, 1999) sexual signal in females may therefore have enforced pair-bonding in humans under certain ecological conditions. Both, synchrony in breeding and concealed ovulation lower male monopolization potential. Aseasonal breeding species with reliable indicators for ovulation fail to lower male mating competition and monopolization potential, making pair-bonding unattractive for males that can still enhance reproductive success by accessing several receptive females at a time. Seasonal breeding with concealed ovulation favors prolonged mate guarding activity by males only for the breeding season. Exclusively in aseasonal breeding species with concealed ovulation females would be able to prolong mate guarding, binding a particular male as potential sire to herself and resulting in paternal care, as suggested for humans (Hawkes, 2004). 


\section{CONCLUSIONS AND FUTURE DIRECTIONS}

My study contributes to the existing evidence of the formation of stable bonds between males and females in large primate groups and their adaptive significance. Additionally, my results extend former knowledge by showing that females may lower the effect of feeding competition with conspecifics when bonding with particular males, and that those bonds are important enough to suffer the costs of competition. To my knowledge, neither direct impact of the strength of dyadic affiliative relationships on ingestion rates, nor the stress response on failing to establish a social bond in competitive situations have been evaluated before. I also provided a theoretical model to disentangle the evolutionary puzzle of the formation of pair-bonds in primates that extends established hypotheses by considering the evolution of permanent pairbonds as an interaction of events rather than the result of a singular change in ecological or physiological terms. More studies on Assamese macaques and other primates are clearly necessary to draw general conclusions. I therefore suggest future directions as follows.

1) My study was based on social bonds in Assamese macaques that already existed at the time of observation. How exactly those bonds are developed and based on which traits is still a gap of knowledge. I did show that, beyond dominance rank, the potential for paternal care has a strong influence on a male's attractiveness. Future research should investigate the development of social bonds from the beginning, by studying the integration of males into social groups and female acceptance after immigration, with focus on male-immature interactions. Moreover, individual personality may play an additional role for the formation of social bonds and should be assessed for both males and females in relation to bonding patterns.

2) Though my study did provide valuable insights into the adaptive significance of opposite-sex social bonds, it remains to be clarified whether reduced feeding competition and enhanced paternal care increase the reproductive success of female Assamese macaques. Concerning the importance of food availability along with other ecological factors, short-term reproductive success may not be meaningful, whereas lifetime (or as close as we may get) reproductive success considering all factors that may influence the outcome should be evaluated.

3) Reproductive success of male Assamese macaques, based on paternity data, should be assessed to identify whether males may be able to actually improve or at least outbalance reproductive success, compared to the effect of dominance rank, when a) bonding with females, and b) providing paternal care to their biological offspring. If so, males may use reproductive strategies that are similar to theories on the evolution of human pair-bonds. 
4) To date, integrating long-term data on both sexes is still lacking throughout studies on non-human primates. The comparability between existing studies is highly demanding and may lead to misinterpretation of results. Primatologists should a) try to use equivalent measurements that are applicable to various ecological, social structure and mating systems, and b) publish unfavorable results to reduce the assumption of false negatives.

5) To unravel the origin of humankind one may want to use integrative approaches comparing different species with their behavioral, physiological and ecological traits. Traits are known to have developed or got lost independently on several branches of the phylogenetic tree. Within the last decades, the fields of primatology and anthropology are already more intertwined. Interdisciplinary studies on non-human and human primates that are not predominantly led by either primatologist or anthropologist may help to solve the puzzle of humanity. 


\section{References}

Abbott, D.H., 1984. Behaviourally mediated suppression of reproduction in female primates. Journal of Zoology. 213, 455-470.

Alberts, S.C., Fitzpatrick, C.L., 2012. Paternal care and the evolution of exaggerated sexual swellings in primates. Behavioral Ecology. 23, 699-706.

Alexander, R.D., Noonan, K.M., 1979. Concealment of ovulation, parental care, and human social evolution. In: Chagnon, N.A., Irons, W.G. (Eds.), Evolutionary Biology and Human Behavior: An Anthropological Perspective. Duxbury Press, North Scituate, pp. 436-453.

Altmann, J., 1974. Observational study of behavior: sampling methods. Behaviour. 49, 227266.

Altmann, J., Alberts, S.C., 2003. Variability in reproductive success viewed from a life-history perspective in baboons. American Journal of Human Biology. 15, 401-409.

Andelman, S.J., 1987. Evolution of concealed ovulation in vervet monkeys (Cercopithecus aethiops). The American Naturalist. 129, 785-799.

Arcadi, A.C., Wrangham, R.W., 1999. Infanticide in chimpanzees: review of cases and a new within-group observation from the Kanyawara study group in Kibale National Park. Primates. 40, 337-351.

Archie, E.A., Tung, J., Clark, M., Altmann, J., Alberts, S.C., 2014. Social affiliation matters: both same-sex and opposite-sex relationships predict survival in wild female baboons. Proceedings of the Royal Society B: Biological Sciences. 281, 20141261.

Aureli, F., Schaffner, C.M., Verpooten, J., Slater, K., Ramos-Fernandez, G., 2006. Raiding parties of male spider monkeys: insights into human warfare. American Journal of Physical Anthropology. 131, 486-497.

Aureli, F., Yates, K., 2010. Distress prevention by grooming others in crested black macaques. Biology Letters. 6, 27-29.

Baayen, R., 2008. Analyzing linguistic data. Cambridge University Press, Cambridge.

Baniel, A., Cowlishaw, G., Huchard, E., 2016. Stability and strength of male-female associations in a promiscuous primate society. Behavioral Ecology and Sociobiology. 70, 761-775.

Barr, D.J., Levy, R., Scheepers, C., Tily, H.J., 2014. Random effects struture for confirmatory hypothesis testing: keep it maximal. Journal of Memory and Language. 68, 1-43.

Barrett, L., Henzi, S.P., Weingrill, T., Lycett, J.E., Hill, R.A., 1999. Market forces predict grooming reciprocity in female baboons. Proceedings of the Royal Society B: Biological Sciences. 266, 665-670.

Bartoń, K., 2013. MuMIn: multi-model inference.

Barton, R.A., 1993. Sociospatial mechanisms of feeding competition in female olive baboons, Papio anubis. Animal Behaviour. 46, 791-802.

Barton, R.A., Byrne, R.W., Whiten, A., 1996. Ecology, feeding competiton and social structure in baboons. Behavioral Ecology and Sociobiology. 38, 321-329.

Barton, R.A., Whiten, A., 1993. Feeding competition among female olive baboons, Papio anubis. Animal Behaviour.

Bates, D., Maechler, M., Bolker, B., Walker, S., 2014. Ime4: Linear mixed-effects models using Eigen and S4.R package version 1.1-7.

Benenson, J.F., 2013. The development of human female competition: allies and adversaries. Philosophical Transactions of the Royal Society B. 368, 20130079.

Bercovitch, F.B., Berard, J.D., 1993. Life history costs and consequences of rapid reproductive maturation in female rhesus macaques. Behavioral Ecology. 32, 103-109.

Berghänel, A., Ostner, J., Schröder, U., Schülke, O., 2011. Social bonds predict future 
cooperation in male Barbary macaques, Macaca sylvanus. Animal Behaviour. 81, 11091116.

Berghänel, A., Schülke, O., Ostner, J., 2010. Coalition formation among Barbary macaque males: the influence of scramble competition. Animal Behaviour. 80, 675-682.

Bergman, T.J., Beehner, J.C., 2015. Measuring social complexity. Animal Behaviour. 103, 203-209.

Bernstein, I.S., Ehardt, C.L., 1985. Agonistic aiding: kinship, rank, age, and sex influences. American Journal of Primatology. 8, 37-52.

Boesch, C., Boesch-Achermann, H., 2000. The chimpanzees of the Taï forest: behavioural ecology and evolution. Oxford University Press, New York.

Bolker, B.M., Brooks, M.E., Clark, C.J., Geange, S.W., Poulsen, J.R., Stevens, M.H.H., White, J.-S.S., 2009. Generalized linear mixed models: a practical guide for ecology and evolution. Trends in Ecology and Evolution. 24, 127-135.

Bongaarts, J., 1980. Does malnutrition affect fecundity? A summary of evidence. Science. 208, 564-569.

Borgeaud, C., Bshary, R., 2015. Wild vervet monkeys trade tolerance and specific coalitionary support for grooming in experimentally induced conflicts. Current Biology. 25, 3011-3016.

Borries, C., Larney, E., Kreetiyutanont, K., Koenig, A., 2002. The diurnal primate community in a dry evergreen forest in Phu Khieo Wildlife Sanctuary, Northeast Thailand. Natural History Bulletin of the Siam Society. 50, 75-88.

Borries, C., Launhardt, K., Epplen, C., Epplen, J.T., Winkler, P., 1999a. Males as infant protectors in Hanuman langurs (Presbytis entellus) living in multimale groups - defence pattern, paternity and sexual behaviour. Behavioral Ecology and Sociobiology. 46, 350356.

Borries, C., Launhardt, K., Epplen, C., Epplen, J.T., Winkler, P., 1999b. DNA analyses support the hypothesis that infanticide is adaptive in langur monkeys. Proceedings of the Royal Society B. 266, 901-904.

Brauch, K., Pfefferle, D., Hodges, K., Möhle, U., Fischer, J., Heistermann, M., 2007. Female sexual behavior and sexual swelling size as potential cues for males to discern the female fertile phase in free-ranging Barbary macaques (Macaca sylvanus) of Gibraltar. Hormones and Behavior. 52, 375-383.

Brewis, A., Meyer, M., 2004. Marital coitus across the life course. Journal of Biosocial Science. 0, 1-20.

Bro-Jørgensen, J., 2002. Overt female mate competition and preference for central males in a lekking antelope. Proceedings of the National Academy of Sciences. 99, 9290-9293.

Broom, M., Borries, C., Koenig, A., 2004. Infanticide and infant defence by males - modelling the conditions in primate multi-male groups. Journal of Theoretical Biology. 231, 261270.

Brown, G.R., Laland, K.N., Borgerhoff Mulder, M., 2009. Bateman's principles and human sex roles. Trends in Ecology and Evolution. 24, 297-304.

Buchan, J.C., Alberts, S.C., Silk, J.B., Altmann, J., 2003. True paternal care in a multi-male primate society. Nature. 425, 179-181.

Buss, D.M., 1988. The evolution of human intrasexual competition: tactics of mate attraction. Journal of Personality and Social Psychology. 54, 616-628.

Buss, D.M., Schmitt, D.P., 1993. Sexual strategies theory: an evolutionary perspective on human mating. Psychological Review. 100, 204-232.

Busse, C.D., Hamilton, W.J., 1981. Infant carrying by male chacma baboons. Science. 212, $1281-1283$.

Byrne, R.W., Whiten, A., Henzi, S.P., 1989. Social relationships of mountain baboons: leadership and affiliation in a non-female-bonded monkey. American Journal of Primatology. 18, 191-207.

Cameron, E.Z., Setsaas, T.H., Linklater, W.L., 2009. Social bonds between unrelated females increase reproductive success in feral horses. Proceedings of the National Academy of Sciences. 106, 13850-13853.

Campbell, A., 2004. Female competition: causes, contraints, contents, and contexts. The 
Journal of Sex Research. 41, 16-26.

Campbell, A., 2013. The evolutionary psychology of women's aggression. Philosophical Transactions of the Royal Society B. 368, 20130078.

Cant, M.A., Young, A.J., 2013. Resolving social conflict among females without overt aggression. Philosophical Transactions of the Royal Society B. 368, 20130076.

Carne, C., Wiper, S., Semple, S., 2011. Reciprocation and interchange of grooming, agonistic support, feeding tolerance, and aggression in semi-free-ranging Barbary macaques. American Journal of Primatology. 73, 1127-1133.

Chapais, B., 1983a. Structure of the birth season relationships among adult male and female rhesus macaques. In: Hinde, R.A. (Ed.), Primate Social Relationships: An Integrated Approach. Blackwell Publications, Oxford, pp. 200-208.

Chapais, B., 1983b. Adaptive aspects of social relationships among adult rhesus monkeys. In: Hinde, R.A. (Ed.), Primate Social Relationships: An Integrated Approach. Blackwell Publications, Oxford, pp. 286-289.

Chapais, B., 1986. Why do adult male and female rhesus monkeys affiliate during the birth season? In: The Cayo Santiago Macaques: History, Behavior, and Biology. University of New York Press, Albany, pp. 173-200.

Chapais, B., 2008. Primeval kinship: How pair-bonding gave birth to human society. Harvard University Press, Cambridge.

Chapais, B., 2013. Monogamy, strongly bonded groups, and the evolution of human social structure. Evolutionary Anthropology. 22, 52-65.

Chapais, B., Berman, C.M., 2004. Kinship and behavior in primates. Oxford University Press, New York.

Chapais, B., Girard, M., Primi, G., 1991. Non-kin alliances, and the stability of matrilineal dominance relations in Japanese macaques. Animal Behaviour. 41, 481-491.

Charpentier, M.J.E., Van Horn, R.C., Altmann, J., Alberts, S.C., 2008. Paternal effects on offspring fitness in a multimale primate society. Proceedings of the National Academy of Sciences. 105, 1988-1992.

Cheney, D.L., Seyfarth, R.M., 1980. Vocal recognition in free-ranging vervet monkeys. Animal Behaviour. 28, 362-367.

Cheney, D.L., Seyfarth, R.M., 1990. The representation of social relations by monkeys. Cognition. 37, 167-196.

Clutton-Brock, T.H., 1988. Reproductive success: studies of individual variation in contrasting breeding systems. University of Chicago Press. University of Chicago Press, Chicago.

Clutton-Brock, T.H., 1989. Review lecture: mammalian mating systems. Proceedings of the Royal Society B: Biological Sciences. 236, 339-372.

Clutton-Brock, T.H., 1991. The evolution of parental care. Princeton University Press, Princeton, New Jersey.

Clutton-Brock, T.H., Harvey, P.H., 1978. Mammals, resources and reproductive strategies. Nature. 273, 191-195.

Clutton-Brock, T.H., Huchard, E., 2013a. Social competition and selection in males and females. Philosophical Transactions of the Royal Society B. 368, 20130074.

Clutton-Brock, T.H., Huchard, E., 2013b. Social competition and its consequences in female mammals. Journal of Zoology. 289, 151-171.

Clutton-Brock, T.H., Parker, G.A., 1995. Punishment in animal societies. Nature. 373, 209216.

Cohen, J., 1992. A power primer. Psychological Bulletin. 112, 155-159.

Colmenares, F., Zaragoza, F., Hernández-Lloreda, M. V, 2002. Grooming and coercion in one-male units of hamadryas baboons: market forces or relationship constraints? Behaviour. 139, 1525-1553.

Connor, R.C., 2007. Dolphin social intelligence: complex alliance relationships in bottlenose dolphins and a consideration of selective environments for extreme brain size evolution in mammals. Philosophical Transactions of the Royal Society B. 362, 587-602.

Connor, R.C., Heithaus, M.R., Barre, L.M., 2001. Complex social structure, alliance stability and mating access in a bottlenose dolphin "super-alliance." Proceedings of the Royal Society B: Biological Sciences. 268, 263-267. 
Cooper, M.A., Bernstein, I.S., 2000. Social grooming in Assamese macaques (Macaca assamensis). American Journal of Primatology. 50, 77-85.

Cords, M., 2002. Friendship among adult female blue monkeys (Cercopithecus mitis). Behaviour. 139, 291-314.

Cords, M., Nikitopoulos, E., 2015. Maternal kin bias in affiliative behavior among wild adult female blue monkeys. American Journal of Primatology. 77, 109-123.

Coxworth, J.E., Kim, P.S., McQueen, J.S., Hawkes, K., 2015. Grandmothering life histories and human pair bonding. Proceedings of the National Academy of Sciences. 112, $11806-11811$.

Crockford, C., Deschner, T., Ziegler, T.E., Wittig, R.M., 2014. Endogenous peripheral oxytocin measures can give insight into the dynamics of social relationships: a review. Frontiers in Behavioral Neuroscience. 8, 1-14.

Crockford, C., Wittig, R.M., Langergraber, K.E., Ziegler, T.E., Zuberbühler, K., Deschner, T., 2013. Urinary oxytocin and social bonding in related and unrelated wild chimpanzees. Proceedings of the Royal Society B: Biological Sciences. 280, 20122765.

Daly, M., Wilson, M.I., 1994. Some differential attributes of lethal assaults on small children by stepfathers versus genetic fathers. Ethology and Sociobiology. 15, 207-217.

Daly, M., Wilson, M.I., 1999. Human evolutionary psychology and animal behaviour. Animal Behaviour. 57, 509-519.

Dammhahn, M., Kappeler, P.M., 2009. Females go where the food is: does the socioecological model explain variation in social organisation of solitary foragers? Behavioral Ecology and Sociobiology. 63, 939-952.

Deag, J.M., 1980. Interactions between males and unweaned Barbary macaques: testing the agonistic buffering hypothesis. Behaviour. 75, 54-80.

de Waal, F.B.M., 1997. The chimpanzee's service economy: food for grooming. Evolution and Human Behavior. 18, 375-386.

Deschner, T., Heistermann, M., Hodges, K., Boesch, C., 2004. Female sexual swelling size, timing of ovulation, and male behavior in wild West African chimpanzees. Hormones and Behavior. 46, 204-215.

Dettmer, A.M., Woodward, R.A., Suomi, S.J., 2015. Reproductive consequences of a matrilineal overthrow in rhesus monkeys. American Journal of Primatology. 77, 346352.

Dittus, W.P.J., Ratnayeke, S.M., 1989. Individual and social behavioral responses to injury in wild toque macaques (Macaca sinica). International Journal of Primatology. 10, 215234.

Dixson, A., Dixson, B., Anderson, M., 2005. Sexual selection and the evolution of visually conspicuous sexually dimorphic traits in male monkeys, apes, and human beings. Annual Review of Sex Research. 16, 1-19.

Dobson, A., 2002. An introduction to Generalized Linear Models. Chapman and Hall, Boca Raton.

Dubuc, C., Brent, L.J.N., Accamando, A.K., Gerald, M.S., MacLarnon, A., Semple, S., Heistermann, M., Engelhardt, A., 2009. Sexual skin color contains information about the timing of the fertile phase in free-ranging Macaca mulatta. International Journal of Primatology. 30, 777-789.

Dunbar, R.I.M., 1992. Neocortex size as a constraint on group size in primates. Journal of Human Evolution. 20, 469-493.

Dunbar, R.I.M., Dunbar, P., 1988. Maternal time budgets of gelada baboons. Animal Behaviour. 36, 970-980.

Dunbar, R.I.M., Shultz, S., 2007. Evolution in the social brain. Science. 317, 1344-1347.

Emlen, S.T., Oring, L.W., 1977. Ecology, sexual selection, and the evolution of mating systems. Science. 197, 215-223.

Engh, A.L., Beehner, J.C., Bergman, T.J., Whitten, P.L., Hoffmeier, R.R., Seyfarth, R.M., Cheney, D.L., 2006. Female hierarchy instability, male immigration and infanticide increase glucocorticoid levels in female chacma baboons. Animal Behaviour. 71, 12271237.

Engh, A.L., Siebert, E.R., Greenberg, D.A., Holekamp, K.E., 2005. Patterns of alliance 
formation and postconflict aggression indicate spotted hyenas recognize third-party relationships. Animal Behaviour. 69, 209-217.

Faer, L.M., Hendriks, A., Abed, R.T., Figueredo, A.J., 2005. The evolutionary psychology of eating disorders: female competition for mates or for status? Psychology and Psychotherapy. 78, 397-417.

Fairbanks, L.A., McGuire, M.T., 1984. Determinants of fecundity and reproductive success in captive vervet monkeys. American Journal of Primatology. 7, 27-38.

Fawcett, T.W., Johnstone, R.A., 2003. Mate choice in the face of costly competition. Behavioral Ecology. 14, 771-779.

Fedigan, L.L.M., Gouzoules, S., Gouzoules, H., Koyama, N., 1986. Lifetime reproductive success in female Japanese macaques. Folia Primatologica. 47, 143-157.

Fernández-Duque, E., Valeggia, C.R., Mendoza, S.P., 2009. The biology of paternal care in human and nonhuman primates. Annual Review of Anthropology. 38, 115-130.

Field, A., 2005. Discovering statistics using SPSS. Sage Publications, London.

Fink, B., Hugill, N., Lange, B.P., 2012. Women's body movements are a potential cue to ovulation. Personality and Individual Differences. 53, 759-763.

Fink, B., Klappauf, D., Brewer, G., Shackelford, T.K., 2014. Female physical characteristics and intra-sexual competition in women. Personality and Individual Differences. 58, 138141.

Fletcher, G.J.O., Simpson, J.A., Campbell, L., Overall, N.C., 2015. Pair-bonding, romantic love, and evolution: the curious case of Homo sapiens. Perspectives on Psychological Science. 10, 20-36.

Foerster, S., McLellan, K., Schroepfer-Walker, K., Murray, C.M., Krupenye, C., Gilby, I.C., Pusey, A.E., 2015. Social bonds in the dispersing sex: partner preferences among adult female chimpanzees. Animal Behaviour. 105, 139-152.

Forstmeier, W., Schielzeth, H., 2011. Cryptic multiple testing in linear models: overestimated effect sizes and the winner's curse. Behavioral Ecology and Sociobiology. 65, 47-55.

Fox, J., Weisberg, S., 2011. An R companion to applied regression, 2nd ed. Sage, Thousand Oaks.

Frank, R., Silk, J.B., 2009. Impatient traders or contingent reciprocators? Evidence for the extended time-course of grooming exchanges in baboons. Behaviour. 146, 1123-1135.

Fraser, O.N., Bugnyar, T., 2012. Reciprocity of agonistic support in ravens. Animal Behaviour. 83, 171-177.

Friedman, H.S., Tucker, J.S., Schwartz, J.E., Tomlinson-Keasey, C., Martin, L.R., Wingard, D.L., Criqui, M.H., 1995. Psychosocial and behavioral predictors of longevity - the aging and death of the "termites." American Psychologist. 50, 69-78.

Fuentes, A., 2002. Patterns and trends in primate pair bonds. International Journal of Primatology. 23, 953-978.

Fürtbauer, I., 2011. The socio-endocrinology of female reproductive strategies in wild Assamese macaques (Macaca assamensis). Göttingen.

Fürtbauer, I., Heistermann, M., Schülke, O., Ostner, J., 2011a. Concealed fertility and extended female sexuality in a non-human primate (Macaca assamensis). PLoS ONE. 6, e23105.

Fürtbauer, I., Heistermann, M., Schülke, O., Ostner, J., 2014. Low female stress hormone levels are predicted by same- or opposite-sex sociality depending on season in wild Assamese macaques. Psychoneuroendocrinology. 48, 19-28.

Fürtbauer, I., Mundry, R., Heistermann, M., Schülke, O., Ostner, J., 2011b. You mate, I mate: macaque females synchronize sex not cycles. PLoS ONE. 6, 6-11.

Fürtbauer, I., Schülke, O., Heistermann, M., Ostner, J., 2010. Reproductive and life history parameters of wild female Macaca assamensis. International Journal of Primatology. 31, 501-517.

Gangestad, S.W., Thornhill, R., 2008. Human oestrus. Proceedings of the Royal Society B: Biological Sciences. 275, 991-1000.

Geary, D.C., 2000. Evolution and proximate expression of human paternal investment. Psychological Bulletin. 126, 55-77.

Gesquiere, L.R., Wango, E.O., Alberts, S.C., Altmann, J., 2007. Mechanisms of sexual 
selection: sexual swellings and estrogen concentrations as fertility indicators and cues for male consort decisions in wild baboons. Hormones and Behavior. 51, 114-125.

Gilby, I.C., Wrangham, R.W., 2008. Association patterns among wild chimpanzees (Pan troglodytes schweinfurthii) reflect sex differences in cooperation. Behavioral Ecology and Sociobiology. 62, 1831-1842.

Goffe, A.S., Zinner, D., Fischer, J., 2016. Sex and friendship in a multilevel society: behavioural patterns and associations between female and male Guinea baboons. Behavioral Ecology and Sociobiology. 70, 323-336.

Gomes, C.M., Mundry, R., Boesch, C., 2009. Long-term reciprocation of grooming in wild West African chimpanzees. Proceedings of the Royal Society B. 276, 699-706.

Gould, L., 1996. Male-female affiliative relationships in naturally occurring ringtailed lemurs (Lemur catta) at the Beza-Mahafaly Reserve, Madagascar. American Journal of Primatology. 39, 63-78.

Grassman, L.I., Tewes, M.E., Silvy, N.J., 2005. Ranging, habitat use and activity patterns of binturong Arctictis binturong and yellow-throated marten Martes flavigula in north-central Thailand. Wildlife Biology. 11, 49-57.

Greenwood, P.J., 1980. Mating systems, philopatry and dispersal in birds and mammals. Animal Behaviour. 28, 1140-1162.

Hämäläinen, A., Heistermann, M., Fenosoa, Z.S.E., Kraus, C., 2014. Evaluating capture stress in wild gray mouse lemurs via repeated fecal sampling: method validation and the influence of prior experience and handling protocols on stress responses. General and Comparative Endocrinology. 195, 68-79.

Hamilton, W.D., 1964. The genetical evolution of social behaviour. II. Journal of Theoretical Biology. 7, 17-52.

Hamilton, W.D., Bulger, J.B., 1990. Natal male baboon rank rises and successful challenges to resident alpha males. Behavioral Ecology and Sociobiology. 26, 357-362.

Hammerstein, P., Noë, R., 2016. Biological trade and markets. Philosophical Transactions of the Royal Society B, Biological Sciences. 371, 20150101.

Haselton, M.G., Mortezaie, M., Pillsworth, E.G., Bleske-Rechek, A., Frederick, D.A., 2007. Ovulatory shifts in human female ornamentation: near ovulation, women dress to impress. Hormones and Behavior. 51, 40-45.

Haunhorst, C.B., Heesen, M., Schülke, O., Ostner, J., 2017. Social bonds with males lower costs of food competition for female Assamese macaques. Animal Behaviour. 125, 5160 .

Haunhorst, C.B., Heistermann, M., Fürtbauer, I., Schülke, O., Ostner, J., n.d. Female competition for "power" and "commitment" in male partners - comparative data from macaques. in prep.

Haunhorst, C.B., Schülke, O., Ostner, J., 2016. Opposite-sex social bonding in wild Assamese macaques. American Journal of Primatology. 78, 872-883.

Hawkes, K., 2004. Mating, parenting, and the evolution of human pair bonds. In: Chapais, B., Berman, C.M. (Eds.), Kinship and Behavior in Primates. Oxford University Press, Oxford, pp. 443-472.

Heesen, M., Rogahn, S., Macdonald, S., Ostner, J., Schülke, O., 2014. Predictors of foodrelated aggression in wild Assamese macaques and the role of conflict avoidance. Behavioral Ecology and Sociobiology. 68, 1829-1841.

Heesen, M., Rogahn, S., Ostner, J., Schülke, O., 2013. Food abundance affects energy intake and reproduction in frugivorous female Assamese macaques. Behavioral Ecology and Sociobiology. 67, 1053-1066.

Heistermann, M., Ademmer, C., Kaumanns, W., 2004. Ovarian cycle and effect of social changes on adrenal and ovarian function in Pygathrix nemaeus. International Journal of Primatology. 25, 689-708.

Heistermann, M., Dittami, J., Reinberg, V., Hodges, J.K., Mo, U., Centre, G.P., 2005. Patterns of anogenital swelling size and their endocrine correlates during ovulatory cycles and early pregnancy in free-ranging Barbary macaques (Macaca sylvanus) of Gibraltar. American Journal of Primatology. 66, 351-368.

Heistermann, M., Palme, R., Ganswindt, A., 2006. Comparison of different 
enzymeimmunoassays for assessment of adrenocortical activity in primates based on fecal analysis. American Journal of Primatology. 273, 257-273.

Hemelrijk, C.K., 1994. Support for being groomed in long-tailed macaques, Macaca fascicularis. Animal Behaviour. 48, 479-481.

Hemelrijk, C.K., Wantia, J., Isler, K., 2008. Female dominance over males in primates: selforganisation and sexual dimorphism. PLoS ONE. 3, e2678.

Hennessy, M.B., Kaiser, S., Sachser, N., 2009. Social buffering of the stress response: diversity, mechanisms, and functions. Frontiers in Neuroendocrinology. 30, 470-482.

Henzi, S.P., Barrett, L., 1999. The value of grooming to female primates. Primates. 40, 4759.

Hewlett, B.S., 1991. Demography and childcare in preindustrial societies. Journal of Anthropological Research. 47, 1-37.

Higham, J.P., Heistermann, M., Saggau, C., Agil, M., Perwitasari-Farajallah, D., Engelhardt, A., 2012. Sexual signalling in female crested macaques and the evolution of primate fertility signals. BMC Evolutionary Biology. 12, 89.

Higham, J.P., Semple, S., MacLarnon, A., Heistermann, M., Ross, C., 2009. Female reproductive signaling, and male mating behavior, in the olive baboon. Hormones and Behavior. 55, 60-67.

Hill, D.A., 1990. Social relationships between adult male and female rhesus macaques: II. non-sexual affiliative behaviour. Primates. 31, 33-50.

Hinde, R.A., 1983. Primate social relationships: An integrated approach. Blackwell Publications, Oxford.

Howie, P.W., McNeilly, A.S., 1982. Effect of breast-feeding patterns on human birth intervals. Journal of Reproduction and Fertility. 65, 545-557.

Hrdy, S.B., 1979. Infanticide among animals: a review, classification, and examination of the implications for the reproductive strategies of females. Ethology and Sociobiology. 1, 13-40.

Huchard, E., Alvergne, A., Féjan, D., Knapp, L.A., Cowlishaw, G., Raymond, M., 2010. More than friends? Behavioural and genetic aspects of heterosexual associations in wild chacma baboons. Behavioral Ecology and Sociobiology. 64, 769-781.

Huchard, E., Charpentier, M.J.E., Marshall, H., King, A.J., Knapp, L.A., Cowlishaw, G., 2013. Paternal effects on access to resources in a promiscuous primate society. Behavioral Ecology. 24, 229-236.

Huchard, E., Cowlishaw, G., 2011. Female-female aggression around mating: an extra cost of sociality in a multimale primate society. Behavioral Ecology. 22, 1003-1011.

Insel, T.R., 2010. The challenge of translation in social neuroscience: a review of oxytocin, vasopressin, and affiliative behavior. Neuron. 65, 768-779.

Isbell, L.A., 1994. Predation on primates: ecological patterns and evolutionary consequences. Evolutionary Anthropology: Issues, News, and Reviews. 3, 61-71.

Josephs, N., Bonnell, T., Dostie, M., Barrett, L., Henzi, S.P., 2016. Working the crowd: sociable vervets benefit by reducing exposure to risk. Behavioral Ecology. 0, 1-7.

Kahlenberg, S.M., Emery Thompson, M., Muller, M.N., Wrangham, R.W., 2008. Immigration costs for female chimpanzees and male protection as an immigrant counterstrategy to intrasexual aggression. Animal Behaviour. 76, 1497-1509.

Kalbitz, J., Ostner, J., Schülke, O., 2016. Strong, equitable and long-term social bonds in the dispersing sex in Assamese macaques. Animal Behaviour. 113, 13-22.

Kaplan, H., Hill, K., Lancaster, J., Hurtado, A.M., 2000. A theory of human life history evolution: diet, intelligence, and longevity. Evolutionary Anthropology. 9, 156-185.

Kaplan, H.S., Hooper, P.L., Gurven, M., 2009. The evolutionary and ecological roots of human social organization. Philosophical Transactions of the Royal Society B: Biological Sciences. 364, 3289-3299.

Kappeler, P.M., van Schaik, C.P., 2002. Evolution of primate social systems. International Journal of Primatology. 23, 707-740.

Kennedy, G.E., 2005. From the ape's dilemma to the weanling's dilemma: early weaning and its evolutionary context. Journal of Human Evolution. 48, 123-145.

Kerhoas, D., Kulik, L., Perwitasari-Farajallah, D., Agil, M., Engelhardt, A., Widdig, A., 2016. 
Mother-male bond, but not paternity, influences male-infant affiliation in wild crested macaques. Behavioral Ecology and Sociobiology. 1-14.

Kerhoas, D., Perwitasari-Farajallah, D., Agil, M., Widdig, A., Engelhardt, A., 2014. Social and ecological factors influencing offspring survival in wild macaques. Behavioral Ecology. 25, 1164-1172.

Kleiman, D.G., Malcolm, J.R., 1981. The evolution of male parental investment in mammals. In: Gubernick, D.J., Klopfer, P.H. (Eds.), Parental Care in Mammals. Plenum Press, New York, pp. 347-387.

Kokko, H., Jennions, M.D., 2008. Parental investment, sexual selection and sex ratios. Journal of Evolutionary Biology. 21, 919-948.

Kudo, H., Dunbar, R.I.M., 2001. Neocortex size and social network size in primates. Animal Behaviour. 62, 711-722.

Kuester, J., Paul, A., 1984. Female reproductive characteristics in semifree-ranging Barbary macaques (Macaca sylvanus). Folia Primatologica. 43, 69-83.

Kuester, J., Paul, A., 1986. Male-infant relationships in semifree-ranging Barbary macaques (Macaca sylvanus) of Affenberg Salem: testing the "male care" hypothesis. American Journal of Primatology. 10, 315-327.

Kulik, L., Muniz, L., Mundry, R., Widdig, A., 2011. Patterns of interventions and the effect of coalitions and sociality on male fitness. Molecular Ecology. 21, 699-714.

Kvarnemo, C., Ahnesjö, I., 1996. The dynamics of operantional sex ratios and competition for mates. Trends in Ecology and Evolution. 11, 404-408.

Langergraber, K., Mitani, J., Vigilant, L., 2009. Kinship and social bonds in female chimpanzees (Pan troglodytes). American Journal of Primatology. 71, 840-851.

Langergraber, K.E., Mitani, J.C., Watts, D.P., Vigilant, L., 2013. Male-female socio-spatial relationships and reproduction in wild chimpanzees. Behavioral Ecology and Sociobiology. 67, 861-873.

Langos, D., Kulik, L., Mundry, R., Widdig, A., 2013. The impact of paternity on male-infant association in a primate with low paternity certainty. Molecular Ecology. 22, 3638-3651.

Lehmann, J., Boesch, C., 2009. Sociality of the dispersing sex: the nature of social bonds in West African female chimpanzees, Pan troglodytes. Animal Behaviour. 77, 377-387.

Lehmann, J., Fickenscher, G., Boesch, C., 2006. Kin biased investment in wild chimpanzees. Behaviour. 143, 931-955.

Leighton, M., Leighton, D.R., 1982. The relationship of size of feeding aggregate to size of food patch: howler monkeys (Alouatta palliata) feeding in Trichilia cipo fruit trees on Barro Colorado Island. Biotropica. 14, 81-90.

Lemasson, A., Palombit, R.A., Jubin, R., 2008. Friendships between males and lactating females in a free-ranging group of olive baboons (Papio hamadryas anubis): evidence from playback experiments. Behavioral Ecology and Sociobiology. 62, 1027-1035.

Lovejoy, C.O., 1981. The origin of man. Science. 211, 341-350.

Lukas, D., Clutton-Brock, T.H., 2013. The evolution of social monogamy in mammals. Science. 341, 526-530.

Lukas, D., Clutton-Brock, T.H., 2014. Evolution of social monogamy in primates is not consistently associated with male infanticide. Proceedings of the National Academy of Sciences. 111, 2014.

MacCormick, H.A., MacNulty, D.R., Bosacker, A.L., Lehman, C., Bailey, A., Collins, D.A., Packer, C., 2012. Male and female aggression: lessons from sex, rank, age, and injury in olive baboons. Behavioral Ecology. 23, 684-691.

Macdonald, S., 2014. Female social relationships among wild Assamese macaques (Macaca assamensis). Göttingen.

Macdonald, S., Schülke, O., Ostner, J., 2013. The absence of grooming for rank-related benefits in female Assamese macaques (Macaca assamensis). International Journal of Primatology. 34, 571-584.

Machanda, Z.P., Gilby, I.C., Wrangham, R.W., 2013. Male-female association patterns among free-ranging chimpanzees (Pan troglodytes schweinfurthii). International Journal of Primatology. 34, 917-938.

Majolo, B., Ventura, R., Schino, G., 2010. Asymmetry and dimensions of relationship quality 
in the Japanese macaque (Macaca fuscata yakui). International Journal of Primatology. $31,736-750$.

Manson, J.H., 1994. Mating patterns, mate choice, and birth season heterosexual relationships in free-ranging rhesus macaques. Primates. 35, 417-433.

Marlowe, F.W., 1999. Male care and mating effort among Hadza foragers. Behavioral Ecology and Sociobiology. 46, 57-64.

Marlowe, F.W., 2000. Paternal investment and the human mating system. Behavioural Processes. 51, 45-61.

Marlowe, F.W., 2003. A critical period for provisioning by Hadza men: implications for pair bonding. Evolution and Human Behavior. 24, 217-229.

Marlowe, F.W., 2004. Is human ovulation concealed? Evidence from conception beliefs in a hunter-gatherer society. Archives of Sexual Behavior. 33, 427-432.

Marlowe, F.W., Berbesque, J.C., 2012. The human operational sex ratio: effects of marriage, concealed ovulation, and menopause on mate competition. Journal of Human Evolution. 63, 834-842.

Marty, P.R., Hodges, K., Agil, M., Engelhardt, A., 2015. Alpha male replacements and delayed dispersal in crested macaques (Macaca nigra). American Journal of Primatology. 9999, 1-8.

Massen, J.J.M., Sterck, E.H.M., 2013. Stability and durability of intra- and intersex social bonds of captive rhesus macaques (Macaca mulatta). International Journal of Primatology. 34, 770-791.

Massen, J.J.M., Sterck, E.H.M., de Vos, H., 2010. Close social associations in animals and humans - functions and mechanisms of friendship. Behaviour. 147, 1379-1412.

Melis, A.P., Hare, B., Tomasello, M., 2006. Engineering cooperation in chimpanzees: tolerance constraints on cooperation. Animal Behaviour. 72, 275-286.

Ménard, N., Segesser, F. Von, Scheffrahn, W., Pastorini, J., 2001. Is male-infant caretaking related to paternity and/or mating activities in wild Barbary macaques (Macaca sylvanus)? Life Sciences. 324, 601-610.

Minge, C., Berghänel, A., Schülke, O., Ostner, J., 2016. Patterns and consequences of maleinfant relationships in wild Assamese macaques (Macaca assamensis). International Journal of Primatology. in press.

Mitani, J.C., 2009. Male chimpanzees form enduring and equitable social bonds. Animal Behaviour. 77, 633-640.

Morris, D., 1967. The naked ape. Bantam Books, London.

Moscovice, L.R., Di Fiore, A., Crockford, C., Kitchen, D.M., Wittig, R., Seyfarth, R.M., Cheney, D.L., 2010. Hedging their bets? Male and female chacma baboons form friendships based on likelihood of paternity. Animal Behaviour. 79, 1007-1015.

Moscovice, L.R., Heesen, M., Di Fiore, A., Seyfarth, R.M., Cheney, D.L., 2009. Paternity alone does not predict long-term investment in juveniles by male baboons. Behavioral Ecology and Sociobiology. 63, 1471-1482.

Nguyen, N., Van Horn, R.C., Alberts, S.C., Altmann, J., 2009. "Friendships" between new mothers and adult males: adaptive benefits and determinants in wild baboons (Papio cynocephalus). Behavioral Ecology and Sociobiology. 63, 1331-1344.

Noë, R., Hammerstein, P., 1995. Biological markets. Trends in Ecology and Evolution. 10, 336-339.

Noë, R., Sluijter, A.A., 1995. Which adult male savanna baboons form coalitions? International Journal of Primatology. 16, 77-104.

Nunn, C.L., 1999. The evolution of exaggerated sexual swellings in primates and the gradedsignal hypothesis. Animal Behaviour. 58, 229-246.

O'Brien, T.G., 1991. Female-male social interactions in wedge-capped capuchin monkeys: benefits and costs of group living. Animal Behaviour. 41, 555-567.

Opie, C., Atkinson, Q.D., Dunbar, R.I.M., Shultz, S., 2013. Male infanticide leads to social monogamy in primates. Proceedings of the National Academy of Sciences. 110, 1332813332.

Opie, C., Atkinson, Q.D., Dunbar, R.I.M., Shultz, S., 2014. Reply to Lukas and Clutton-Brock: Infanticide still drives primate monogamy. Proceedings of the National Academy of 
Sciences. 111, E1675-E1675.

Ostner, J., Heistermann, M., Schülke, O., 2008. Dominance, aggression and physiological stress in wild male Assamese macaques (Macaca assamensis). Hormones and Behavior. 54, 613-619.

Ostner, J., Heistermann, M., Schülke, O., 2011. Male competition and its hormonal correlates in Assamese macaques (Macaca assamensis). Hormones and Behavior. 59, 105-113.

Ostner, J., Kappeler, P.M., 1999. Central males instead of multiple pairs in redfronted lemurs, Eulemur fulvus rufus (Primates, Lemuridae)? Animal Behaviour. 58, 1069-1078.

Ostner, J., Schülke, O., 2014. The evolution of social bonds in primate males. Behaviour. 151, 871-906.

Ostner, J., Vigilant, L., Bhagavatula, J., Franz, M., Schülke, O., 2013. Stable heterosexual associations in a promiscuous primate. Animal Behaviour. 86, 623-631.

Palombit, R.A., 1999. Infanticide and the evolution of pair bonds in nonhuman primates. Evolutionary Anthropology. 7, 117-129.

Palombit, R.A., 2000. Infanticide and the evolution of male-female bonds in animals. In: van Schaik, C.P., Janson, C.H. (Eds.), Infanticide by Males and Its Implications. Cambridge University Press, Cambridge, pp. 239-268.

Palombit, R.A., 2009. "Friendship" with males: a female counterstrategy to infanticide in chacma baboons of the Okavango delta. In: Muller, M.N., Wrangham, R.W. (Eds.), Sexual Coercion in Primates and Humans: An Evolutionary Perspective on Male Aggression against Females. Harvard University Press, Cambridge, pp. 377-409.

Palombit, R.A., Cheney, D.L., Seyfarth, R.M., 2001. Female-female competition for male friends in wild chacma baboons, Papio cynocephalus ursinus. Animal Behaviour. 61, 1159-1171.

Palombit, R.A., Seyfarth, R.M., Cheney, D.L., 1997. The adaptive value of "friendships" to female baboons: experimental and observational evidence. Animal Behaviour. 54, 599614.

Parker, G.A., 1974. Courtship persistence and female-guarding as male time investment strategies. Behaviour. 482, 157-184.

Parrish, J.K., Hammer, W.M., Prewitt, C.T., 1997. Introduction - from individuals to aggregations: unifying properties, global frameworks, and the holy grails of congegration. In: Parrish, J.K., Hammer, W.M. (Eds.), Animal Groups in Three Dimensions: How Species Aggregate. Cambridge University Press, Cambridge, pp. 113.

Paul, A., Kuester, J., Arnemann, J., 1996. The sociobiology of male-infant interactions in Barbary macaques, Macaca sylvanus. Animal Behaviour. 51, 155-170.

Paul, A., Preuschoft, S., van Schaik, C.P., 2000. The other side of the coin: infanticide and the evolution of affiliative male-infant interactions. In: Van Schaik, C.P., Janson, C.H. (Eds.), Infanticide by Males and Its Implications. Cambridge University Press, Cambridge, pp. 269-292.

Pereira, M.E., McGlynn, C.A., 1997. Special relationships instead of female dominance for redfronted lemurs, Eulemur fulvus rufus. American Journal of Primatology. 43, 239-258.

Perry, S., 1997. Male-female social relationships in wild white-faced capuchins (Cebus capucinus). Behaviour. 134, 477-510.

Perry, S., Manson, J.H., Muniz, L., Gros-Louis, J., Vigilant, L., 2008. Kin-biased social behaviour in wild adult female white-faced capuchins, Cebus capucinus. Animal Behaviour. 76, 187-199.

Phillips, K.A., 1995. Resource patch size and flexible foraging in white-faced capuchins (Cebus capucinus). International Journal of Primatology. 16, 509-519.

Plavcan, J.M., 2001. Sexual dimorphism in primate evolution. Yearbook of Physical Anthropology. 44, 25-53.

Pradhan, G., van Schaik, C.P., 2008. Infanticide-driven intersexual conflict over matings in primates and its effects on social organization. Behaviour. 145, 251-275.

Pusey, A.E., 1987. Sex-biased dispersal and inbreeding avoidance in birds and mammals. Trends in Ecology and Evolution. 2, 295-299.

Pusey, A.E., Schroepfer-Walker, K., 2013. Female competition in chimpanzees. 
Philosophical Transactions of the Royal Society B. 368, 20130077.

Quinlan, R.J., 2007. Human parental effort and environmental risk. Proceedings of the Royal Society B. 274, 121-125.

Quinlan, R.J., Quinlan, M.B., 2007. Evolutionary ecology of human pair-bonds: cross-cultural tests of alternative hypotheses. Cross-Cultural Research. 41, 149-169.

Quinlan, R.J., Quinlan, M.B., 2008. Human lactation, pair-bonds, and alloparents: a crosscultural analysis. Human Nature. 19, 87-102.

Quinn, G., Keough, M., 2002. Experimental designs and data analysis for biologists. Cambridge University Press, Cambridge.

Ratnayeke, S., 1994. The behavior of postreproductive females in a wild population of toque macaques (Macaca sinica) in Sri Lanka. International Journal of Primatology. 15, 445469.

Reed, C., O'Brien, T.G., Kinnaird, M.F., 1997. Male social behavior and dominance hierarchy in the Sulawesi crested black macaque. International Journal of Primatology. 18, 247260.

Rimbach, R., Link, A., Heistermann, M., Gómez-posada, C., Galvis, N., Heymann, E.W., 2013. Effects of logging, hunting, and forest fragment size on physiological stress levels of two sympatric ateline primates in Colombia. Conservation Physiology. 1, 1-11.

Roberts, S.C., Havlicek, J., Flegr, J., Hruskova, M., Little, A.C., Jones, B.C., Perrett, D.I., Petrie, M., 2004. Female facial attractiveness increases during the fertile phase of the menstrual cycle. Proceedings of the Royal Society B. 271, S270-S272.

Robinson, J.G., 1982. Intrasexual competition and mate choice in primates. American Journal of Primatology. Supp 1, 131-144.

Romero, T., Aureli, F., 2008a. Reciprocity of support in coatis (Nasua nasua). Journal of Comparative Psychology. 122, 19-25.

Romero, T., Aureli, F., 2008b. Reciprocity of support in coatis (Nasua nasua). Journal of comparative psychology. 122, 19-25.

Rose, L.M., 1994. Benefits and costs of resident males to females in white-faced capuchins, Cebus capucinus. American Journal of Primatology. 32, 235-248.

Ross, H.E., Young, L.J., 2009. Oxytocin and the neural mechanisms regulating social cognition and affiliative behavior. Frontiers in Neuroendocrinology. 30, 534-547.

Rosvall, K.A., 2011. Intrasexual competition in females: evidence for sexual selection? Behavioral Ecology. 22, 1131-1140.

Sadleir, R.M.F.S., 1969. The role of nutrition in the reproduction of wild mammals. Journal of Reproduction and Fertility Supplement. 6, 39-48.

Salmon, C., Crawford, C.B., Walters, S., 2008. Anorexic behavior, female competition and stress: developing the female competition stress test. Evolutionary Psychology. 6, 96112.

Sapolsky, R.M., Ray, J.C., 1989. Styles of dominance and their endocrine correlates among wild olive baboons (Papio anubis). American Journal of Primatology. 18, 1-13.

Schino, G., Aureli, F., 2009. Reciprocal altruism in primates: partner choice, cognition, and emotions. Advances in the Study of Behavior. 39, 45-69.

Schino, G., Aureli, F., 2010a. The relative roles of kinship and reciprocity in explaining primate altruism. Ecology Letters. 13, 45-50.

Schino, G., Aureli, F., 2010b. A few misunderstandings about reciprocal altruism. Communicative and Integrative Biology. 3, 561-563.

Schino, G., Aureli, F., 2010c. Primate reciprocity and its cognitive requirements. Evolutionary Anthropology. 19, 130-135.

Schmid, V.S., De Vries, H., 2013. Finding a dominance order most consistent with a linear hierarchy: an improved algorithm for the I\&SI method. Animal Behaviour. 86, 10971105.

Schmitt, D.P., 2005. Sociosexuality from Argentina to Zimbabwe: a 48-nation study of sex, culture, and strategies of human mating. Behavioral and Brain Sciences. 28, 247-311.

Schneider, J.E., 2004. Energy balance and reproduction. Physiology and Behavior. 81, 289317.

Schülke, O., Bhagavatula, J., Vigilant, L., Ostner, J., 2010. Social bonds enhance 
reproductive success in male macaques. Current Biology. 20, 2207-2210.

Schülke, O., Ostner, J., 2012. Ecological and social influences on sociality. In: The Evolution of Primate Societies. pp. 195-219.

Schülke, O., Pesek, D., Whitman, B.J., Ostner, J., 2011. Ecology of Assamese macaques (Macaca assamensis) at Phu Khieo Wildlife Sanctuary, Thailand. Journal of Wildlife Thailand. 18, 1-15.

Schülke, O., Wenzel, S., Ostner, J., 2013. Paternal relatedness predicts the strength of social bonds among female rhesus macaques. PLoS ONE. 8, e59789.

Sear, R., Mace, R., 2008. Who keeps children alive? A review of the effects of kin on child survival. Evolution and Human Behavior. 29, 1-18.

Setchell, J.M., Lee, P.C., Wickings, E.J., Dixson, A.F., 2002. Reproductive parameters and maternal investment in mandrills (Mandrillus sphinx). International Journal of Primatology. 23, 51-68.

Seyfarth, R.M., 1977. A model of social grooming among adult female monkeys. Journal of Theoretical Biology. 65, 671-698.

Seyfarth, R.M., 1978a. Social relationships among adult male and female baboons. II. Behaviour throughout the female reproductive cycle. Behaviour. 64, 227-247.

Seyfarth, R.M., 1978b. Social relationships among adult male and female baboons. I. Behaviour during sexual consortship. Behaviour. 64, 204-226.

Seyfarth, R.M., 1980. The distribution of grooming and related behaviors among adult female vervet monkeys. Animal Behaviour. 28, 798-813.

Seyfarth, R.M., Cheney, D.L., 1984. Grooming, alliances and reciprocal altruism in vervet monkeys. Nature. 308, 541-543.

Shultz, S., Opie, C., Atkinson, Q.D., 2011. Stepwise evolution of stable sociality in primates. Nature. 479, 219-222.

Shutt, K., MacLarnon, A., Heistermann, M., Semple, S., 2007. Grooming in Barbary macaques: better to give than to receive? Biology Letters. 3, 231-233.

Shutt, K., Setchell, J., Heistermann, M., 2012. Non-invasive monitoring of physiological stress in the western lowland gorila (Gorilla gorilla gorilla): validation of a faecal glucocorticoid assay and methods for practical application in the field. General and Comparative Endocrinology. 179, 167-177.

Silk, J.B., 1994. Social relationships of male bonnet macaques: male bonding in a matrilineal society. Behaviour. 130, 271-291.

Silk, J.B., 1999. Male bonnet macaques use information about third-party rank relationships to recruit allies. Animal Behaviour. 58, 45-51.

Silk, J.B., 2002. Using the'F'-word in primatology. Behaviour. 139, 421-446.

Silk, J.B., 2007. Social components of fitness in primate groups. Science. 317, 1347-1351.

Silk, J.B., Alberts, S.C., Altmann, J., 2003. Social bonds of female baboons enhance infant survival. Science. 302, 1231-1234.

Silk, J.B., Alberts, S.C., Altmann, J., 2004. Patterns of coalition formation by adult female baboons in Amboseli, Kenya. Animal Behaviour. 67, 573-582.

Silk, J.B., Alberts, S.C., Altmann, J., 2006a. Social relationships among adult female baboons (Papio cynocephalus) II. Variation in the quality and stability of social bonds. Behavioral Ecology and Sociobiology. 61, 197-204.

Silk, J.B., Alberts, S.C., Altmann, J., Cheney, D.L., Seyfarth, R.M., 2012. Stability of partner choice among female baboons. Animal Behaviour. 83, 1511-1518.

Silk, J.B., Altmann, J., Alberts, S.C., 2006b. Social relationships among adult female baboons (Papio cynocephalus) I. Variation in the strength of social bonds. Behavioral Ecology and Sociobiology. 61, 183-195.

Silk, J.B., Beehner, J.C., Bergman, T.J., Crockford, C., Engh, A.L., Moscovice, L.R., Wittig, R.M., Seyfarth, R.M., Cheney, D.L., 2009. The benefits of social capital: close social bonds among female baboons enhance offspring survival. Proceedings of the Royal Society B. 276, 3099-3104.

Silk, J.B., Beehner, J.C., Bergman, T.J., Crockford, C., Engh, A.L., Moscovice, L.R., Wittig, R.M., Seyfarth, R.M., Cheney, D.L., 2010a. Female chacma baboons form strong, equitable, and enduring social bonds. Behavioral Ecology and Sociobiology. 64, 1733- 
1747.

Silk, J.B., Beehner, J.C., Bergman, T.J., Crockford, C., Engh, A.L., Moscovice, L.R., Wittig, R.M., Seyfarth, R.M., Cheney, D.L., 2010b. Strong and consistent social bonds enhance the longevity of female baboons. Current Biology. 20, 1359-1361.

Silk, J.B., Cheney, D.L., Seyfarth, R.M., 2013. A practical guide to the study of social relationships. Evolutionary Anthropology. 22, 213-225.

Silk, J.B., Clark-Wheatley, C.B., Rodman, P.S., Samuels, A., 1981. Differential reproductive success and facultative adjustment of sex ratios among captive female bonnet macaques (Macaca radiata). Animal Behaviour. 29, 1106-1120.

Small, M.F., 1990a. Promiscuity in Barbary Macaques (Macaca sylvanus). American Journal of Primatology. 20, 267-282.

Small, M.F., 1990b. Alloparental behaviour in Barbary macaques, Macaca sylvanus. Animal Behaviour. 39, 297-306.

Smith, J.E., Van Horn, R.C., Powning, K.S., Cole, A.R., Graham, K.E., Memenis, S.K., Holekamp, K.E., 2010. Evolutionary forces favoring intragroup coalitions among spotted hyenas and other animals. Behavioral Ecology. 21, 284-303.

Smuts, B.B., 1983. Dynamics of "special relationships" between adult male and female olive baboons. In: Hinde, R.A. (Ed.), Primate Social Relationships: An Integrated Approach. Blackwell Publications, Oxford, pp. 112-116.

Smuts, B.B., 1985. Sex and friendship in baboons. Aldine Publ, New York.

Smuts, B.B., Gubernick, D.J., 1992. Male-infant relationships in nonhuman primates: paternal investment or mating effort? In: Hewlett, B.S. (Ed.), Father-Child Relations: Cultural and Biosocial Contexts. Aldine Publ, New York, pp. 1-30.

Smuts, B.B., Watanabe, J.M., 1990. Social relationships and ritualized greetings in adult male baboons (Papio cynocephalus anubis). International Journal of Primatology. 11, 147-172.

Sterck, E.H.M., Watts, D.P., van Schaik, C.P., 1997. The evolution of female social relationships in nonhuman primates. Behavioral Ecology and Sociobiology. 41, 291309.

Stockley, P., Bro-Jørgensen, J., 2011. Female competition and its evolutionary consequences in mammals. Biological Reviews. 86, 341-366.

Stockley, P., Campbell, A., 2013. Female competition and aggression: interdisciplinary perspectives. Philosophical Transactions of the Royal Society B. 368, 20130073.

Strassmann, B.I., 1981. Sexual selection, paternal care, and concealed ovulation in humans. Ethology and Sociobiology. 2, 31-40.

Strier, K.B., 1989. Effects of food patch size on feeding associations in muriquis (Brachyteles arachnoides). Folia Primatol. 52, 70-77.

Strum, S.C., Western, J.D., 1982. Variations in fecundity with age and environment in olive baboons (Papio anubis). American Journal of Primatology. 3, 61-76.

Sugiyama, Y., Ohsawa, H., 1982. Population dynamics of Japanese monkeys with special reference to the effect of artificial feeding. Folia Primatologica. 39, 238-263.

Sukmak, M., Wajjwalku, W., Ostner, J., Schülke, O., 2014. Dominance rank, female reproductive synchrony, and male reproductive skew in wild Assamese macaques. Behavioral Ecology and Sociobiology. 68, 1097-1108.

Surbeck, M., Mundry, R., Hohmann, G., 2011. Mothers matter! Maternal support, dominance status and mating success in male bonobos (Pan paniscus). Proceedings of the Royal Society B. $278,590-598$.

Swedell, L., Plummer, T., 2012. A papionin multilevel society as a model for hominin social evolution. International Journal of Primatology. 33, 1165-1193.

Takahata, Y., 1982. Social relationships between male and female Japanese macaques. Primates. 23, 1-23.

Taub, D.M., 1980. Testing the "agonistic buffering" hypothesis. Behavioral ecology and sociobiology. 6, 187-197.

Thierry, B., Iwaniuk, A.N., Pellis, S.M., 2000. The Influence of phylogeny on the social behaviour of macaques (primates: Cercopithecidae, genus Macaca). Ethology. 106, 713-728. 
Tiddi, B., Aureli, F., Polizzi Di Sorrentino, E., Janson, C.H., Schino, G., 2011. Grooming for tolerance? Two mechanisms of exchange in wild tufted capuchin monkeys. Behavioral Ecology. 22, 663-669.

Tobias, J.A., Montgomerie, R., Lyon, B.E., 2012. The evolution of female ornaments and weaponry: social selection, sexual selection and ecological competition. Philosophical Transactions of the Royal Society B: Biological Sciences. 367, 2274-2293.

Trivers, R.L., 1972. Parental investment and sexual selection. In: Campbell, B. (Ed.), Sexual Selection and the Descent of Man 1871-1971. Aldine Publ, Chicago, pp. 136-179.

Tsukahara, T., 1990. Initiation and solicitation in male-female grooming in a wild Japanese macaque troop on Yakushima island. Primates. 31, 147-156.

Tutin, C.E.G., 1979. Mating patterns and reproductive strategies in a community of wild chimpanzees (Pan troglodytes schweinfurthii). Behavioral Ecology and Sociobiology. 6 , 29-38.

Vaillancourt, T., 2013. Do human females use indirect aggression as an intrasexual competition strategy? Philosophical Transactions of the Royal Society B. 368, 20130080.

van Noordwijk, M.A., van Schaik, C.P., 1987. Competition among female long-tailed macaques, Macaca fascicularis. Animal Behaviour. 35, 577-589.

van Schaik, C.P., 1989. The ecology of social relationships amongst female primates. In: Standen, V., Foley, R.A. (Eds.), Comparative Primate Socioecology: The Behavioural Ecology of Humans and Other Mammals. Blackwell Publications, Oxford, pp. 195-218.

van Schaik, C.P., 1996. Social evolution in primates: the role of ecological factors and male behaviour. Proceedings of the British Academy. 88, 9-31.

van Schaik, C.P., Dunbar, R.I.M., 1990. The evolution of monogamy in large primates: a new hypothesis and some crucial tests. Behaviour. 115, 30-62.

van Schaik, C.P., Kappeler, P.M., 1997. Infanticide risk and the evolution of male-female association in primates. Proceedings. Biological sciences / The Royal Society. 264, 1687-1694.

van Schaik, C.P., Paul, A., 1996. Male care in primates: does it ever reflect paternity? Evolutionary Anthropology. 5, 152-156.

van Schaik, C.P., van Noordwijk, M.A., 1985. Interannual variability in fruit abundance and the reproductive seasonality in Sumatran Long-tailed macaques (Macaca fascularis). Journal of Zoology. 206, 533-549.

Villiers, M.S., Richardson, P.R.K., Jaarsveld, A.S., 2003. Patterns of coalition formation and spatial association in a social carnivore, the African wild dog (Lycaon pictus). Journal of Zoology. 260, 377-389.

Vogel, E.R., Janson, C.H., 2007. Predicting the frequency of food-related agonism in whitefaced capuchin monkeys (Cebus capucinus), using a novel focal-tree method. American Journal of Primatology. 69, 533-550.

Wasser, S.K., Starling, A.K., 1988. Proximate and ultimate causes of reproductive suppression among female yellow baboons at Mikumi National Park, Tanzania. American Journal of Primatology. 16, 97-121.

Watanabe, K., Mori, A., Kawai, M., 1992. Characteristic features of the reproduction of Koshima monkeys, Macaca fuscata fuscata: a summary of thirty-four years of observation. Primates. 33, 1-32.

Watts, D.P., 1998. Coalitionary mate guarding by male chimpanzees at Ngogo, Kibale National Park, Uganda. Behavioral Ecology and Sociobiology. 44, 43-55.

Watts, D.P., 2002. Reciprocity and interchange in the social relationships of wild male chimpanzees. Behaviour. 139, 343-370.

Watts, D.P., 2010. Dominance, power, and politics in nonhuman and human primates. In: Kappeler, P.M., Silk, J.B. (Eds.), Mind the Gap: Tracing the Origins of Human Universals. Springer, Heidelberg, pp. 109-138.

Weingrill, T., Lycett, J.E., Barrett, L., Hill, R.A., Henzi, S.P., 2003. Male consortship behaviour in chacma baboons: the role of demographic factors and female conceptive probabilities. Behaviour. 140, 405-427.

Weingrill, T., Willems, E.P., Zimmermann, N., Steinmetz, H., Heistermann, M., 2011. 
Species-specific patterns in fecal glucocorticoid and androgen levels in zoo-living orangutans (Pongo spp.). General and Comparative Endocrinology. 172, 446-457.

Weinrich, J.D., 1977. Human sociobiology: pair-bonding and resource predictability (effects of social class and race). Behavioral Ecology and Sociobiology. 2, 91-118.

Weyher, A.H., Phillips-Conroy, J.E., Fourrier, M.S., Jolly, C.J., 2014. Male-driven grooming bouts in mixed-sex dyads of Kinda baboons (<i>Papio kindae<i/>). Folia Primatol. 85, 178-191.

Whitten, P.L., 1983. Diet and dominance among female vervet monkeys (Cercopithecus aethiops). American Journal of Primatology. 5, 139-159.

Wickham, H., Chang, W., 2016. ggplot2.

Widdig, A., Nürnberg, P., Krawczak, M., Streich, W.J., Bercovitch, F.B., 2001. Paternal relatedness and age proximity regulate social relationships among adult female rhesus macaques. Proceedings of the National Academy of Sciences. 98, 13769-13773.

Widdig, A., Streich, W.J., Tembrock, G., 2000. Coalition formation among male Barbary macaques (Macaca sylvanus). American Journal of Primatology. 50, 37-51.

Williams, M., Grajales, C.A.G., Kurkiewicz, D., 2013. Assumptions of multiple regression: correcting two misconceptions. Practical Assessment, Research and Evaluation. 18, 114.

Winking, J., Gurven, M., Kaplan, H., 2011. Father death and adult success among the Tsimane: implications for marriage and divorce. Evolution and Human Behavior. 32, 7989.

Wittig, R.M., Crockford, C., Langergraber, K.E., Zuberbühler, K., 2014. Triadic social interactions operate across time: a field experiment with wild chimpanzees. Proceedings of the Royal Society B. 281, 20133155.

Wittig, R.M., Crockford, C., Seyfarth, R.M., Cheney, D.L., 2007. Vocal alliances in chacma baboons (Papio hamadryas ursinus). Behavioral Ecology and Sociobiology. 61, 899909.

Wittig, R.M., Crockford, C., Weltring, A., Langergraber, K.E., Deschner, T., Zuberbühler, K., 2016. Social support reduces stress hormone levels in wild chimpanzees across stressful events and everyday affiliations. Nature Communications. 7, 13361.

Woodroffe, R., Vincent, A., 1994. Mother's little helpers: patterns of male care in mammals. Trends in Ecology and Evolution. 9, 294-297.

Wrangham, R.W., 1980. An ecological model of female-bonded primate groups. Behaviour. 75, 262-300.

Wright, P.C., 1990. Patterns of paternal care in primates. International Journal of Primatology. 11, 89-102.

Young, C., Majolo, B., Heistermann, M., Schülke, O., Ostner, J., 2014a. Responses to social and environmental stress are attenuated by strong male bonds in wild macaques.

Proceedings of the National Academy of Sciences. 111, 18195-18200.

Young, C., Majolo, B., Schülke, O., Ostner, J., 2014b. Male social bonds predict partner recruitment in cooperative aggression in wild Barbary macaques. Animal Behaviour. 80, 675-682.

Zabel, C.J., Glickman, S.E., Frank, L.G., Woodmansee, K.B., Keppel, G., 1992. Coalition formation in a colony of prepubertal spotted hyenas. In: Harcourt, A., de Waal, F.B.M. (Eds.), Coalitions and Alliances in Humans and Other Animals. Oxford University Press, New York, pp. 113-135. 


\section{Acknowledgements}

I need to thank many people that contributed the one or other way to the completion of my thesis. It has been three and a half years of sweat, frustration, and tears, but also of overwhelming joy, confidence, and the development of differentiated, stable and more or less balanced relationships within and between sexes. During this time, I learned to let go and to hold on, to give up and to pursue, to reach my limits and go beyond. I did not do it alone, nor would I have been able to do so. I had an army of people to cheer me on, keep me grounded, and share my success - may it have been ever so little. I will try to thank those people. I am sure I will forget some and give others maybe not enough credit. If this may be the case, it was not done intentionally.

First, and most importantly, I want to thank Julia Ostner and Oliver Schülke to have given me the opportunity to work on the project. You supported me from the beginning, gave me your trust, and let me be independent. I thank you for the advice, the supervision, so many meetings that extended the intended two minutes to several hours; the many times I ran into your office with some supposedly amazing results, and that you still took me serious in my overexcitement. Oliver, you were always as excited as I was, pushing me to peak performance and maybe the only other person that actually believed in me finishing in time. Julia, you were the reasonable mind, made me step back, and reconsider my excitement to take a broader and more realistic view of my doing. Thanks to both of you for being the right combination of excitement and cautiousness. Thanks to both of you to have supported me beyond my thesis in the development of my personality. You taught me more than you may be aware of and shaped me to be a better person!

I want to thank the members of my BeCog thesis committee, Dietmar Zinner and Tanya Behne, for valuable input and advice from an interdisciplinary perspective. Further, I thank Peter Kappeler and Bernhard Fink to agree on being part of the committee in my oral defense. Many thanks go to Chris Schloegl who organized so many things for me regarding my enrollment in both BeCog and the university while I was already in Thailand and for later advice regarding job opportunities. I also want to thank Rebecca Jürgens for organization and help regarding my handing in of the thesis.

I am very grateful to the National Research Council of Thailand (NRCT) and the Department of National Parks, Wildlife and Plant Conservation (DNP) for the permission to conduct this study and for all the support (permit 0004.3/3618, 0002.3/2647, 0002/17, 0002/2424). Many thanks goes to Kanjana Nitaya, Kitti Kreetiyutanont, Mongkul Kumsuk, 
Jarupol Prabnasuk and Thanee Wongnak for supporting our project and making things easy in Phu Khieo Wildlife Sanctuary. I thank A. Koenig and C. Borries, who developed the field site at Huai Mai Sot Yai. Special thanks go to all members of team macaque for collecting so much behavioral data and so many hormone samples over the years. I want to particularly thank Wet Nueorngshiyos, Piya Saaisawasthikul, and Tawut (Wat) Wisate. Wet collected very detailed data on females for me for over a year and oftentimes cheered me up in the forest when I was again not able to identify monkeys in the huge fig trees. I thank Piya for his positive attitude, the handling of organizational things in the park, his efforts to print posters, deal with complicated visa affairs, and the updates on soccer world cup and general global and German politics. Wat heroically filled in for Wet in terms of data collection in the last few months, was my nurse when I suffered allergic shocks, supplied me with meds and good advice and made me remember how privileged I am to work with these monkeys. You gave me your trust as a friend, thank you.

My study would not have been possible in the current version without the support of my colleagues. I thank Ines Fürtbauer, Sebastian Roghan, Marlies Heesen, Sally Macdonald, Andreas Berghänel and Josefine Kalbitz for the contribution of data to my study. I got the full advantage of working on a long-term project, drawing on the resources and results that existed already. Specifically, I want to point out two people whose previous dedication made my life so much easier and the progress of my study ever so much faster: Josefine Kalbitz and Andreas Berghänel. Andreas, you provided the brains that created macros in Excel unheard of before (at least by me). Your "Aufdrösler" made it possible for me to prepare all data for analysis while I was still in the field. Your programs made data collection and progressing a delight that took hardly any time. Because of the time you spent on programming, I could calculate indices from raw data within a day. I cannot thank you enough! Josi, you were the one who had all the bad luck and somehow lived through it with a smile on your face. You were the first to make me feel like home in Thailand, you were the one who made Andreas work all night on programming macros, and you were the one with a friendly ear when I needed a trustworthy person to talk. You are more than just a colleague - you are friend. We spent days and nights, talking about so many different things and just never stopped talking. Your cheerful disposition even in the worst situations, your ability to see the beauty of things that appear ugly to anyone else kept me on track many more times than you may imagine.

My other colleagues in Göttingen I did not forget, but I will not name everyone. Thanks to all of you for sharing the time with me; for wild dances at DPZ Christmas parties and unforgettable nights in Hanoi and Rome. Special thanks go to Pascal for bugging me with statistics and raising my confidence and to Luca and Flávia for NOT talking science. I want to thank Eva for bearing me walking past in the office a million times without ever complaining, and Steffi for our "almost" regular girls' nights with Josi. To Cédric I am grateful for many conversations (serious or not), advice (professional or not), and to many times he challenged 
me (positive or not). I am grateful to Anja Ebenau (who did read everything twice), Cédric Girard-Buttoz, Josefine Kalbitz, Markus Port, and Pascal Marty for comments on earlier drafts of the thesis.

A very dear thank goes to two special people that contributed to my work in very different ways; one before the study even started, the other since the day I boarded the plane to Thailand. Maryjka Blaszczyk gave me the chance to work as research and field assistant on her PhD study in South Africa after my diploma thesis. Maryjka, from the very first day I arrived in Bloemfontein you made me feel welcome and appreciated. We shared a room, the sundowner, and stories about our families. I found a friendship that survives spatial segregation and months without talking. In that respect, it will always be (oh oh) you and me. Professionally, you taught me the basics by letting myself try and providing advice when I needed it. You endured my nagging questions, asked my advice and gave me responsibility that most field assistants may not get. You helped me with my applications, without ever changing my personal style. Because of the time I spent in Soetdoring with you and the vervets, I felt confident enough to master a PhD in primatology.

The other special person kept me going so many times while I was in Thailand and beyond the time in the field to the present day. Anja Ebenau was the manager of the campsite at Phu Khieo during my field study. Anja, the two of us were probably not "love on first sight", but definitely at the third repacking of our check-in luggage at the Etihad counter at Frankfurt airport. Ever since, we were a team that was sometimes brutally honest with each other, but always loyal, covering each other's backs. You welcomed me home at night in Thailand and settled my frustration by teaching me that "monkeys live in trees". You let me be who I am and never questioned my character, always bearing my temper, never cowering in spite of it, but rather keeping your ground. We trusted each other (and still do) far beyond work. Fate threw us together, but it will not separate us!

I want to thank some very special friends that somehow contributed to this work, probably without even knowing. First, to my best friend Milli who was always a rock I could lean on, for more than 30 years now. She listened to things she didn't understand and somehow still managed to give me sound advice. To Bene I am grateful for ever so many funny chats that made me laugh, even after a full day in the forest without any data to show for and for encouraging me in going beyond - in so many ways. I will always love you! Thanks to Eva and Anika for still being my friends, and somehow dealing with my inability to keep in touch. To my aunt Margit, whose interest and fascination may be unheard of, for the many picturesquely supporting mails. I thank Malte for tearing me away from the computer when I needed it the most, to clear my head in outdoor activities. Finally, I want to thank Caro for her unexpected friendship - in next life I want to meet earlier.

Last but not least, my family as a whole (and it is a whole bunch) deserves more gratitude on my side than I will ever be able to pronounce. We may have our fights, but at the 


\section{Acknowledgements}

end of the day, we will always be one. None of you ever doubted me, questioned my intentions, or my chances for success. You had my back, wherever I went, from the moment I was born. Bettina, thanks for endless Thai nights on Skype. You made me realize how important family is, and that nothing should ever break it apart, no matter what. Franzi, thanks for dealing with me in the past few months, for hugging me, for letting me ramble, and for acting as if you would understand what the heck I was talking about. Kath and Peppi, you followed me around the world and made me see the beauty of a country I have been living in for so long. You made me leave my computer at home and relax when I dearly needed it, for two beautiful weeks in Scotland. The both of you shared my frustration, my joy, my excitement, my disappointment, and my success. To Peppi goes some very special thanks for so many hours on the phone in Thailand (if the weather was fine), for appreciating the importance of an average of 1 when calculating the CSI, and for letting me be part of her own PhD thesis, when I lost faith in myself. Mama \& Papa, you raised me to never take things for granted, to work hard for the things I want, and to not give up in case of failure. You taught me to know my limits and overcome them. You were always a shoulder to cry on, a friendly ear, a tower of strength. You supported me throughout my life, you let me go so many times, send me a bunch of sweets on the way - and let me come back home. I may not have called a place "home", but wherever you were. Every single one of you contributed a part to this work. 


\title{
Christine Barbara Haunhorst
}

\author{
Curriculum vitae
}

\section{Address}

Regerstr. 22

55127 Mainz

Germany

Email: c.haunhorst@gmail.com

Date of birth: 22 ${ }^{\text {nd }}$ March 1984

\section{ACADEMIC CAREER}

2013-2016 PhD Behavioral Ecology, University of Göttingen

Thesis title: "Evolutionary origin of the human pair-bond: the adaptive significance of male-female relationships in wild Assamese macaques, Thailand"

Supervisor: Prof. Dr. Julia Ostner

2011-2012 Field assistant position: Temperament and social niche specialization in South African vervet monkeys Department of Anthropology, Center for the Study of Human Origins, New York University

Supervisor: Dr. Maria B. Blaszczyk

2010-2011 Diploma thesis: "The puku antelope and its competitors in Kafue National Park and surrounding Game Management Areas, Zambia"

Department of animal ecology and tropical biology at University of Würzburg and Zoologisches Forschungsmuseum Alexander Koenig, Bonn (Zoological Research-Museum) Supervisor: Prof. Dr. Karl-Eduard Linsenmair

$2005-2011 \quad$ Studies of Biology, University of Würzburg (2007-2011) and University of Mainz (2005-2007) 


\section{GRANTS}

$2013-2016$

Studienstiftung des Deutschen Volkes

Full-time funding of dissertation project

2008

DAAD; Funding for practical courses in foreign countries (Panama)

\section{CONFERENCE CONTRIBUTIONS}

2016

2015

2015

„How female Assamese macaques benefit from oppositesex social bonds"

Ethological Society Meeting; Göttingen

"Costs of competition for access to males in wild female

Assamese macaques" (Poster)

European Federation of Primatology Meeting; Rome

"The nature of male-female relationships in wild Assamese macaques"

Deutschen Gesellschaft für Primatologie Meeting; Leipzig

2014 „Male-female bonds in Wild Assamese macaques“ (Poster) International Primatological Society Meeting; Hanoi

\section{SOCIETY MEMBERSHIP}

2014

Deutsche Gesellschaft für Primatologie

2014

International Primatological Society

\section{PUBLICATIONS}

Haunhorst CB, Schülke O \& Ostner J (2016): Opposite-sex social bonding in wild Assamese macaques. American Journal of Primatology

Haunhorst CB, Heesen M, Ostner J \& Schülke O (2017): Social bonds with males lower costs of competition for female Assamese macaques.

Animal Behaviour

Haunhorst CB, Heistermann M, Fürtbauer I, Schülke O \& Ostner J (to be submitted):

Female competition for 'power' and 'commitment' in male partners - Assamese macaques as a test case. Journal of Human Evolution 


\section{Declaration}

I hereby declare that all parts of my thesis were written by myself. Assistance of third parties was only accepted if scientifically justifiable and acceptable in regards to the examination regulations. Assistance or contributions to the individual chapters are indicated and all sources have been quoted.

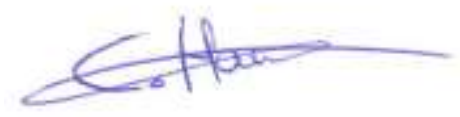

Christine Barbara Haunhorst 